\title{
THE LEAST COMMON MULTIPLE OF CONSECUTIVE QUADRATIC PROGRESSION TERMS
}

\author{
SHAOFANG HONG AND GUOYOU QIAN
}

\begin{abstract}
Let $k$ be an arbitrary given positive integer and let $f(x) \in \mathbb{Z}[x]$ be a quadratic polynomial with $a$ and $D$ as its leading coefficient and discriminant, respectively. Associated to the least common multiple $\operatorname{lcm}_{0 \leq i \leq k}\{f(n+i)\}$ of any $k+1$ consecutive terms in the quadratic progression $\{f(n)\}_{n \in \mathbb{N}^{*}}$, we define the function $g_{k, f}(n):=\left(\prod_{i=0}^{k}|f(n+i)|\right) / \operatorname{lcm}_{0 \leq i \leq k}\{f(n+i)\}$ for all integers $n \in \mathbb{N}^{*} \backslash Z_{k, f}$, where $Z_{k, f}:=\bigcup_{i=0}^{k}\left\{n \in \mathbb{N}^{*}: f(n+i)=0\right\}$. In this paper, we first show that $g_{k, f}$ is eventually periodic if and only if $D \neq a^{2} i^{2}$ for all integers $i$ with $1 \leq i \leq k$. Consequently, we develop a detailed $p$-adic analysis of $g_{k, f}$ and determine its smallest period. Finally, we obtain asymptotic formulas of $\log \operatorname{lcm}_{0 \leq i \leq k}\{f(n+i)\}$ for all quadratic polynomials $f$ as $n$ goes to infinity.
\end{abstract}

\section{Introduction and the statements of the main results}

The study of least common multiple of consecutive positive integers was initiated by Chebyshev 3 for the first significant attempt to prove prime number theorem. Motivated by Chebyshev's work, one naturally expects to investigate the least common multiple of consecutive terms in any given sequence of positive integers. For the least common multiple of the first $n$ terms of a given sequence of positive integers, some results were obtained by several authors. Hanson [8] and Nair [20] got the upper and lower bound of $\operatorname{lcm}_{1 \leq i \leq n}\{i\}$ respectively. Bateman, Kalb and Stenger [2] obtained an asymptotic formula for the least common multiple of arithmetic progressions. Farhi [5] [6] and Farhi and Kane [7] studied the least common multiple of some finite sequences of integers. Hong and Feng [10, Hong and Yang [15, Kane and Kominers [17] and Wu, Tan and Hong 24 . investigated the least common multiple of finite arithmetic progressions. Hong, Qian and Tan 13 obtained an asymptotic estimate for the least common multiple of a sequence of products of linear polynomials. Qian and Hong [22 got an asymptotic formula for the least common multiple of consecutive arithmetic progression terms.

The investigation of periodic arithmetic functions has been a common topic in number theory for a long time. The readers are refereed to [1] and [19] for the related background information. When investigating the least common multiple $\operatorname{lcm}_{0 \leq i \leq k}\{n+i\}$ of any $k+1$ consecutive integers with $k$ being a fixed positive integer, Farhi [ 6 introduced the arithmetic function $\bar{g}_{k}$ defined for positive integer $n$ by $\bar{g}_{k}(n):=\frac{\prod_{i=0}^{k}(n+i)}{\operatorname{lcm}_{0 \leq i \leq k}\{n+i\}}$. Farhi showed that $\bar{g}_{k}$ is periodic with $k$ ! as its period. Let $\bar{P}_{k}$ be the smallest period of

Date: September 24, 2018.

2000 Mathematics Subject Classification. Primary 11B83, 11A05, 11N13.

Key words and phrases. quadratic progression, least common multiple, quadratic congruence, $p$-adic analysis, the smallest period.

The work was supported partially by National Science Foundation of China Grant \#11371260, by the Ph.D. Programs Foundation of Ministry of Education of China Grant \#20100181110073 and by the Postdoctoral Science Foundation of China Grant \#2013M530109. 
$\bar{g}_{k}$. Then $\bar{P}_{k} \mid k$ !. At the end of [6], Farhi posed the open problem of determining the smallest period $\bar{P}_{k}$. Hong and Yang [14] improved the period $k$ ! to $\operatorname{lcm}_{1 \leq i \leq k}\{i\}$ and proposed a conjecture stating that $\frac{\operatorname{lcm}_{1 \leq i \leq k+1}\{i\}}{k+1}$ divides $\bar{P}_{k}$. Farhi and Kane [7] proved the Hong-Yang conjecture and finally determined the exact value of $\bar{P}_{k}$. Throughout, let $\mathbb{Q}, \mathbb{Z}$ and $\mathbb{N}$ denote the field of rational numbers, the ring of integers and the set of nonnegative integers, respectively. Define $\mathbb{N}^{*}:=\mathbb{N} \backslash\{0\}$. Let $b \in \mathbb{N}$ and $a, k \in \mathbb{N}^{*}$. Define $L_{k}:=\operatorname{lcm}_{1 \leq i \leq k}\{i\}$. Hong and Qian [12] defined the arithmetic function $g_{k, a, b}: \mathbb{N}^{*} \longrightarrow \mathbb{N}^{*}$ by $g_{k, a, b}(n):=\frac{\prod_{i=0}^{k}(b+(n+i) a)}{\operatorname{lcm}_{0 \leq i \leq k}\{b+(n+i) a\}}$, and proved that $g_{k, a, b}$ is periodic and determined the exact value of the smallest period of $g_{k, a, b}$. Let $f(x)$ be a quadratic polynomial with integer coefficients. Associated to $\operatorname{lcm}_{0 \leq i \leq k}\{f(n+i)\}$, we define the function $g_{k, f}$ for all positive integers $n \in \mathbb{N}^{*} \backslash Z_{k, f}$ by

$$
g_{k, f}(n):=\frac{\prod_{i=0}^{k}|f(n+i)|}{\operatorname{lcm}_{0 \leq i \leq k}\{f(n+i)\}},
$$

where $Z_{k, f}:=\bigcup_{i=0}^{k}\left\{n \in \mathbb{N}^{*}: f(n+i)=0\right\}$. Recall that an arithmetic function $\alpha$ is called eventually periodic if there are positive integers $t_{0}$ and $n_{0}$ such that $\alpha\left(n+t_{0}\right)=\alpha(n)$ holds for all integers $n \geq n_{0}$. If $f(x)=x^{2}+1$, then it is proved in [23] that $g_{k, f}$ is periodic and also the smallest period is determined there. We can easily find that there are quadratic polynomials $f$ such that $g_{k, f}$ is not eventually periodic. Therefore one naturally asks the following interesting question.

Problem 1.1. 9] Let $f(x)$ be a polynomial of degree two with integer coefficients and $g_{k, f}$ be defined as in (1.1). Characterize $f(x)$ such that $g_{k, f}$ is eventually periodic. If $g_{k, f}$ is eventually periodic, what is the smallest period of $g_{k, f}$ ?

In this paper, we mainly focus on the least common multiple of any $k+1$ consecutive terms in the quadratic progression. Our main goal is to study Problem 1.1. First we use a well-known identity of Hua 16 to determine all the quadratic integer polynomials $f$ such that $g_{k, f}$ is eventually periodic. Then we transfer the computation of the smallest period of $g_{k, f}$ into the computation of the local smallest periods. To determine the local smallest periods, we will first investigate the minimal distance among the roots of quadratic congruences and then develop a detailed local analysis. To state the main results, let's introduce and recall some notation. As usual, for any prime number $p$, we let $\nu_{p}$ be the normalized $p$-adic valuation of $\mathbb{Q}$, i.e., $\nu_{p}(s)=t$ if $p^{t} \| s$. Let $\operatorname{gcd}(s, t)$ denote the greatest common divisor of any integers $s$ and $t$. For any real number $x$, by $\lfloor x\rfloor$ we denote the largest integer no more than $x$. Throughout this paper, we always let $a \geq 1$ and $f(x)=a x^{2}+b x+c$ be any given quadratic polynomial with integer coefficients, and let $D:=b^{2}-4 a c$ be the discriminant of $f$. Define $D_{4}:=\frac{D}{4^{\left.\frac{\nu_{2}(D)}{2}\right\rfloor}}$ and $D_{p}:=\frac{D}{p^{\nu_{p}(D)}}$ for any odd prime $p$. Then $D_{4}$ is equal to $\frac{D}{2^{\nu_{2}(D)}}$ if $\nu_{2}(D)$ is even, and equals $\frac{2 D}{2^{\nu_{2}(D)}}$ if $\nu_{2}(D)$ is odd. So $D_{4} \not \equiv 0(\bmod 4), \nu_{2}(D)$ and $D_{4}$ hold the reverse parity. Let $(\dot{\bar{p}})$ denote the Legendre symbol. For any positive integer $k$, we define $B_{k}:=\operatorname{lcm}_{1 \leq i \leq k}\left\{i\left(a^{2} i^{2}-D\right)\right\}$ and

$$
A_{k}:=\frac{B_{k}}{\xi_{2}\left(\prod_{p \neq 2, p \mid \operatorname{gcd}(a, b)} p^{\nu_{p}\left(B_{k}\right)}\right)\left(\prod_{p \nmid 2 a D,\left(\frac{D}{p}\right)=-1} p^{\nu_{p}\left(B_{k}\right)}\right)\left(\prod_{p \nmid 2 a, p \mid D} \eta_{p}\right)}
$$

where 
$(1.3)$

$\xi_{2}=\left\{\begin{aligned} 1, & \text { if } 2 \mid a, 2 \nmid b \text { and } \nu_{2}(k+1)<\nu_{2}\left(B_{k}\right), \\ 2^{2 \nu_{2}\left(L_{k}\right)}, & \text { if } 2 \nmid a, k<2^{\left\lfloor\frac{\nu_{2}(D)}{2}\right\rfloor} \text { and } \nu_{2}(k+1)<\nu_{2}\left(L_{k}\right), \\ 2^{\nu_{2}\left(B_{k}\right)-\left\lfloor\frac{\nu_{2}(D)}{2}\right\rfloor,}, & \text { if } 2 \nmid a, k \geq 2^{\left\lfloor\frac{\nu_{2}(D)}{2}\right\rfloor}, D_{4} \neq \equiv \quad(\bmod 8) \text { and } \nu_{2}(k+1)<\left\lfloor\frac{\nu_{2}(D)}{2}\right\rfloor, \\ 2^{\nu_{2}(D)+1}, & \text { if } 2 \nmid a, k \geq 2^{\left\lfloor\frac{\nu_{2}(D)}{2}\right\rfloor} \text { and } D_{4} \equiv 1 \quad(\bmod 8), \\ 2^{\nu_{2}\left(B_{k}\right)}, & \text { otherwise }\end{aligned}\right.$

and

$$
\eta_{p}=\left\{\begin{aligned}
p^{2 \nu_{p}\left(L_{k}\right)}, & \text { if } k<p^{\left\lceil\frac{\nu_{p}(D)}{2}\right\rceil} \text { and } \nu_{p}(k+1)<\nu_{p}\left(L_{k}\right), \\
p^{\nu_{p}\left(B_{k}\right)-\left\lceil\frac{\nu_{p}(D)}{2}\right\rceil,}, & \text { if } k \geq p^{\left\lceil\frac{\nu_{p}(D)}{2}\right\rceil}, \nu_{p}(k+1)<\left\lceil\frac{\nu_{p}(D)}{2}\right\rceil \\
& \text { and either } 2 \nmid \nu_{p}(D) \text { or }\left(\frac{D_{p}}{p}\right)=-1, \\
p^{\nu_{p}(D)}, & \text { if } k \geq p^{\left\lceil\frac{\nu_{p}(D)}{2}\right\rceil}, \nu_{p}(k+1)<\nu_{p}\left(B_{k}\right)-\nu_{p}(D), \\
& 2 \mid \nu_{p}(D) \text { and }\left(\frac{D_{p}}{p}\right)=1, \\
p^{\nu_{p}\left(B_{k}\right),}, & \text { otherwise. }
\end{aligned}\right.
$$

We can now state the first main result of this paper as follows.

Theorem 1.2. Let $f(x)$ be a polynomial of degree two with integer coefficients and $k$ be a positive integer. Let $g_{k, f}$ be defined as in (1.1). Then $g_{k, f}$ is eventually periodic if and only if $D \neq a^{2} i^{2}$ for all integers $i$ with $1 \leq i \leq k$. If $g_{k, f}$ is eventually periodic, then its smallest period is equal to $A_{k}$ except that $\nu_{p}(k+1) \geq \nu_{p}\left(A_{k}\right) \geq 1$ for at most one odd prime $p$ such that either $p \mid a$ and $p \nmid b$ or $p \nmid 2 a D$ and $\left(\frac{D}{p}\right)=1$, in which case its smallest period equals $A_{k} / p^{\nu_{p}\left(A_{k}\right)}$.

Therefore Theorem 1.2 answers completely Problem 1.1 .

By [12, we know that for any integers $a \geq 1$ and $b$, one has $\log \operatorname{lcm}_{0 \leq i \leq k}\{a(n+i)+b\} \sim$ $(k+1) \log n$ as $n$ goes to infinity. Now using Theorem 1.2. we can deduce the following asymptotic estimates of $\log \operatorname{lcm}_{0 \leq i \leq k}\{f(n+i)\}$ for all quadratic polynomials $f$ with integer coefficients as $n$ tends to infinity. This is the second main result of this paper.

Theorem 1.3. Let $k$ be a positive integer. Then $\log \operatorname{lcm}_{0 \leq i \leq k}\{f(n+i)\}=C_{k, D} \log n+$ $o(\log n)$, where $C_{k, D}=2(k+1)$ if $D \neq a^{2} i^{2}$ for all integers $i$ with $1 \leq i \leq k$, and $C_{k, D}=k+i_{0}+1$ if $D=a^{2} i_{0}^{2}$ for some integer $i_{0}$ with $1 \leq i_{0} \leq k$.

This paper is organized as follows. We first show in Section 2 that $g_{k, f}$ is eventually periodic if and only if $D \neq a^{2} i^{2}$ for all integers $i$ with $1 \leq i \leq k$. Subsequently, we give a formula which factors the global smallest period $P_{k, f}$ into the product of the local smallest periods $P_{p, k, f}$. Then in Section 3, we study the structure of the roots of quadratic congruences and introduce the concept of the minimal distance among the roots of quadratic congruences. Consequently, we develop some arithmetic properties of the minimal distance. In Section 4, we provide a detailed $p$-adic analysis of $g_{k, f}$, and then using the arithmetic results obtained in Section 3, we arrive at explicit formulas of the local snallest periods $P_{p, k, f}$. In Section 5 , by using the results presented in Section 4, we show Theorem 1.2. Some examples are also given in Section 5 to demonstrate the validity of Theorem 1.2. Finally, in Section 6, we give the proof of Theorem 1.3 .

\section{A characterization on $f$ such that $g_{k, f}$ is eventually periodic}

Throughout this section, we always let $f$ be a quadratic primitive polynomial with integer coefficients (i.e. the greatest common divisor of all the coefficients of $f$ is 1 ). We 
first characterize all the quadratic primitive polynomials $f$ with integer coefficients such that $g_{k, f}$ is eventually periodic. Subsequently, we transfer the smallest period problem into a local analysis problem. We begin with the following result which answers the first part of Problem 1.1 .

Theorem 2.1. Let $k$ be a positive integer. The function $g_{k, f}$ is eventually periodic if and only if $D \neq a^{2} i^{2}$ for all integers $i$ with $1 \leq i \leq k$. Furthermore, if $g_{k, f}$ is eventually periodic, then $B_{k}$ is its period.

Proof. First we show the necessity part. Let $g_{k, f}$ be eventually periodic. Suppose that $D=a^{2} i_{0}^{2}$ for some integer $i_{0}$ with $1 \leq i_{0} \leq k$. Then $f(x)$ is reducible. We may let $f(x)=$ $a x^{2}+b x+c:=\left(a_{1} x+b_{1}\right)\left(a_{2} x+b_{2}\right)$ with $\operatorname{gcd}\left(a_{1}, b_{1}\right)=\operatorname{gcd}\left(a_{2}, b_{2}\right)=1$ and $a_{1}, a_{2} \in \mathbb{N}^{*}$. Then $D=\left(a_{2} b_{1}-a_{1} b_{2}\right)^{2}=a_{1}^{2} a_{2}^{2} i_{0}^{2}$. In other words, we have $a_{2} b_{1}-a_{1} b_{2}= \pm a_{1} a_{2} i_{0}$ and so $a_{2}\left(b_{1} \pm a_{1} i_{0}\right)=a_{1} b_{2}$. It follows that $a_{1}=a_{2}$ and $b_{2}=b_{1} \pm a_{1} i_{0}$. So we can write $f$ as $f(x)=\left(a_{1} x+b_{1}\right)\left(a_{1} x+b_{1} \pm a_{1} i_{0}\right)$.

If $b_{2}=b_{1}+a_{1} i_{0}$, then for any positive integer $n, a_{1} n+b_{1}+a_{1} i_{0}$ divides $\frac{\left|f(n) f\left(n+i_{0}\right)\right|}{\operatorname{lcm}\left(f(n), f\left(n+i_{0}\right)\right)}$. Hence $\left(a_{1} n+b_{1}+a_{1} i_{0}\right) \mid g_{k, f}(n)$ which implies that $g_{k, f}(n) \geq a_{1} n+b_{1}+a_{1} i_{0}$.

If $b_{2}=b_{1}-a_{1} i_{0}$, we obtain that $\left(a_{1} n+b_{1}\right) \mid g_{k, f}(n)$ and $g_{k, f}(n) \geq a_{1} n+b_{1}$. Thus $g_{k, f}(n)$ tends to infinity as $n$ tends to infinity. This is impossible since $g_{k, f}$ is eventually periodic implies that $g_{k, f}(n)$ is bounded. The necessity part is proved.

Consequently, we show the sufficiency part. Let $D \neq a^{2} i^{2}$ for all integers $i$ with $1 \leq i \leq k$. Then we have $B_{k}=\operatorname{lcm}_{1 \leq i \leq k}\left\{i\left(a^{2} i^{2}-D\right)\right\} \neq 0$. For any given positive integer $n \in \mathbb{N}^{*} \backslash Z_{k, f}$, we derive from the identity $(2 a n+3 a j-a i+b) f(n+i)-(2 a n+$ $3 a i-a j+b) f(n+j)=(j-i)\left(a^{2}(j-i)^{2}-D\right)$ that

$$
\operatorname{gcd}(f(n+i), f(n+j)) \mid \operatorname{lcm}_{0 \leq i<j \leq k}\left\{(j-i)\left(a^{2}(j-i)^{2}-D\right)\right\}=B_{k} .
$$

It follows that $\operatorname{gcd}(f(n+i), f(n+j)) \mid f\left(n+i \pm B_{k}\right)$ and $\operatorname{gcd}(f(n+i), f(n+j)) \mid f\left(n+j \pm B_{k}\right)$. Thus we have $\operatorname{gcd}(f(n+i), f(n+j)) \mid \operatorname{gcd}\left(f\left(n+B_{k}+i\right), f\left(n+B_{k}+j\right)\right)$ and

$$
\operatorname{gcd}(f(n+i), f(n+j)) \mid \operatorname{gcd}\left(f\left(n-B_{k}+i\right), f\left(n-B_{k}+j\right)\right) .
$$

Replacing $n$ by $n+B_{k}$ in (2.2), one gets $\operatorname{gcd}\left(f\left(n+B_{k}+i\right), f\left(n+B_{k}+j\right)\right) \mid \operatorname{gcd}(f(n+$ $i), f(n+j))$. Therefore $\operatorname{gcd}(f(n+i), f(n+j))=\operatorname{gcd}\left(f\left(n+i+B_{k}\right), f\left(n+j+B_{k}\right)\right)$ for any positive integer $n \in \mathbb{N}^{*} \backslash Z_{k, f}$ and any integers $i, j$ with $0 \leq i<j \leq k$. But Theorem 7.3 in Chapter 1 of [16] (see Page 11 of [16]) tells us that

$$
g_{k, f}(n)=\prod_{r=1}^{k} \prod_{0 \leq i_{0}<\ldots<i_{r} \leq k}\left(\operatorname{gcd}\left(f\left(n+i_{0}\right), \ldots, f\left(n+i_{r}\right)\right)\right)^{(-1)^{r-1}}
$$

and

$$
g_{k, f}\left(n+B_{k}\right)=\prod_{r=1}^{k} \prod_{0 \leq i_{0}<\ldots<i_{r} \leq k}\left(\operatorname{gcd}\left(f\left(n+B_{k}+i_{0}\right), \ldots, f\left(n+B_{k}+i_{r}\right)\right)\right)^{(-1)^{r-1}} .
$$

Thus $g_{k, f}\left(n+B_{k}\right)=g_{k, f}(n)$ for any positive integer $n \in \mathbb{N}^{*} \backslash Z_{k, f}$, and $g_{k, f}$ is eventually periodic with $B_{k}$ as its period. The proof of Theorem 2.1 is complete.

It should be pointed out that the condition that $D \neq a^{2} i^{2}$ for all integers $i$ with $1 \leq i \leq k$ is equivalent to saying that $f(x)$ and $f(x+i)$ are relatively prime in $\mathbb{Q}[x]$ for all integers $i$ with $1 \leq i \leq k$. So Theorem 2.1 tells us that $g_{k, f}$ is eventually periodic if and only if $f(x)$ and $\operatorname{lcm}_{1 \leq i \leq k}\{f(x+i)\}$ are relatively prime in $\mathbb{Q}[x]$. After giving a characterization on $f$ so that $g_{k, f}$ is eventually periodic, we now turn our attention 
to determining the smallest period of $g_{k, f}$. Our basic idea is to transfer the problem into a local analysis problem such that we can provide a local analysis to $g_{k, f}$. It is clear that only when $f$ is reducible, $Z_{k, f}$ is not empty. In this case, for all $n \in Z_{k, f}$, we can always find a positive integer $a_{0}$ such that $n+a_{0} B_{k} \in \mathbb{N}^{*} \backslash Z_{k, f}$. By defining $g_{k, f}(n):=g_{k, f}\left(n+a_{0} B_{k}\right)$ for each $n \in Z_{k, f}$, we get the extended periodic arithmetic function $g_{k, f}: \mathbb{N}^{*} \longrightarrow \mathbb{N}^{*}$ with $B_{k}$ as its period. In what follows, when mentioning $g_{k, f}$, we will mean the extended periodic arithmetic function $g_{k, f}$. For any given prime $p$, we define the arithmetic function $g_{p, k, f}$ for any positive integer $n$ by $g_{p, k, f}(n):=v_{p}\left(g_{k, f}(n)\right)$. If $g_{k, f}$ is periodic with $P_{k, f}$ as its smallest period, then $g_{p, k, f}$ is periodic and $P_{k, f}$ is a period of $g_{p, k, f}$. Let $P_{p, k, f}$ be the smallest period of $g_{p, k, f}$. Then $P_{p, k, f} \mid P_{k, f}$. The following result factors the global smallest period $P_{k, f}$ into the product of the local smallest periods $P_{p, k, f}$.

Lemma 2.2. For any prime $p, P_{p, k, f}$ divides $p^{\nu_{p}\left(B_{k}\right)}$. Further, $P_{k, f}=\prod_{p \mid B_{k}} P_{p, k, f}$.

Proof. For any positive integer $n$ and any two integers $i, j$ with $0 \leq i<j \leq k$, we get by (2.1) that $v_{p}(\operatorname{gcd}(f(n+i), f(n+j)))=\min \left\{\nu_{p}(f(n+i)), \nu_{p}(f(n+j))\right\} \leq \nu_{p}\left(B_{k}\right)$, which means that $\nu_{p}(f(n+i)) \leq \nu_{p}\left(B_{k}\right)$ or $\nu_{p}(f(n+j)) \leq \nu_{p}\left(B_{k}\right)$. Therefore $\nu_{p}(f(n+i)) \leq$ $\nu_{p}\left(f\left(n+i \pm p^{\nu_{p}\left(B_{k}\right)}\right)\right)$ or $\nu_{p}(f(n+j)) \leq \nu_{p}\left(f\left(n+j \pm p^{\nu_{p}\left(B_{k}\right)}\right)\right)$. Hence we derive that

$$
\begin{aligned}
\nu_{p}(\operatorname{gcd}(f(n+i), f(n+j))) & \leq \min \left(\nu_{p}\left(f\left(n+i+p^{\nu_{p}\left(B_{k}\right)}\right)\right), \nu_{p}\left(f\left(n+j+p^{\nu_{p}\left(B_{k}\right)}\right)\right)\right) \\
& =v_{p}\left(\operatorname{gcd}\left(f\left(n+i+p^{\nu_{p}\left(B_{k}\right)}\right), f\left(n+j+p^{\nu_{p}\left(B_{k}\right)}\right)\right)\right)
\end{aligned}
$$

and

$$
\nu_{p}(\operatorname{gcd}(f(n+i), f(n+j))) \leq \nu_{p}\left(\operatorname{gcd}\left(f\left(n+i-p^{\nu_{p}\left(B_{k}\right)}\right), f\left(n+j-p^{\nu_{p}\left(B_{k}\right)}\right)\right)\right) .
$$

Replacing $n$ by $n+p^{\nu_{p}\left(B_{k}\right)}$ in (2.3) , we obtain $\nu_{p}\left(\operatorname{gcd}\left(f\left(n+i+p^{\nu_{p}\left(B_{k}\right)}\right), f(n+j+\right.\right.$ $\left.\left.\left.p^{\nu_{p}\left(B_{k}\right)}\right)\right)\right) \leq \nu_{p}(\operatorname{gcd}(f(n+i), f(n+j)))$. Thus we have that $v_{p}\left(\operatorname{gcd}\left(f\left(n+i+p^{\nu_{p}\left(B_{k}\right)}\right), f(n+\right.\right.$ $\left.\left.\left.j+p^{\nu_{p}\left(B_{k}\right)}\right)\right)\right)=v_{p}(\operatorname{gcd}(f(n+i), f(n+j)))$ for any positive integer $n$ and any two integers $0 \leq i<j \leq k$. It then follows from Theorem 7.3 in Chapter 1 of [16 that $g_{p, k, f}(n)=g_{p, k, f}\left(n+p^{\nu_{p}\left(B_{k}\right)}\right)$ for any positive integer $n$. Hence we get that $p^{\nu_{p}\left(B_{k}\right)}$ is a period of $g_{p, k, f}$ and thus $P_{p, k, f} \mid p^{\nu_{p}\left(B_{k}\right)}$. It tells us that $P_{p, k, f}$ are relatively prime for different prime numbers $p$ and $P_{p, k, f}=1$ for all primes $p \nmid B_{k}$.

On the other hand, since $P_{q, k, f} \mid P_{k, f}$ for each prime $q$, we have $\prod_{\text {prime } q \mid B_{k}} P_{q, k, f} \mid P_{k, f}$. Since $P_{q, k, f}=1$ for all primes $q \nmid R_{k}$, we have for each prime $p$ and any positive integer $n$ that $\nu_{p}\left(g_{k, f}\left(n+\prod_{\text {prime } q \mid B_{k}} P_{q, k, f}\right)\right)=\nu_{p}\left(g_{k, f}(n)\right)$, which implies that $\prod_{p \mid B_{k}} P_{p, k, f}$ is a period of $g_{k, f}$ and $P_{k, f} \mid \prod_{p \mid B_{k}} P_{p, k, f}$. Thus the desired result follows immediately. So Lemma 2.2 is proved.

By Lemma 2.2, to get the global smallest period $P_{k, f}$, it is sufficient to determine the exact value of the local smallest period $P_{p, k, f}$ for all the primes $p \mid B_{k}$. The remaining part of the paper will devote to establishing such a local analysis.

\section{Minimal distance among the roots of a quadratic congruence}

Throughout this section, we let $f(x)=a x^{2}+b x+c$ be an arbitrary primitive quadratic polynomial with integer coefficients and let $p$ denote a prime. A natural question is to determine the roots of the congruence $f(x) \equiv 0\left(\bmod p^{e}\right)$ and to investigate the relation among distinct roots. Note that the number of roots of the congruence $x^{2} \equiv n\left(\bmod p^{e}\right)$ is given in [16, where $e$ and $n$ are positive integers such that $p \nmid n$. Also notice that 
the problem of distribution of roots of quadratic congruences to prime modulus was investigated by Duke, Friedlander, Iwaniec [4. Our concern here is the structure of the roots of the congruence $f(x) \equiv 0\left(\bmod p^{e}\right)$.

For any given nonnegative integer $e$, by $S\left(f, p^{e}\right)$ we denote the set of solutions $x$ with $1 \leq x \leq p^{e}$ of the congruence $f(x) \equiv 0\left(\bmod p^{e}\right)$. Evidently, $S\left(f, p^{0}\right)=\{1\}$. Throughout, for any $x \in \mathbb{Z}_{p}$, the ring of $p$-adic integers, by $\langle x\rangle_{p^{e}}$ we mean an integer between 1 and $p^{e}$ such that $\langle x\rangle_{p^{e}} \equiv x\left(\bmod p^{e}\right)$. We begin with the following lemma.

Lemma 3.1. Let e be a positive integer and let $p$ be a prime such that $p \mid a$. Then $S\left(f, p^{e}\right)$ is empty if $p \mid b$, and equals $\left\{\left\langle s_{p}\right\rangle_{p^{e}}\right\}$ if $p \nmid b$, where $s_{p}$ is the unique solution of the equation $f(x)=0$ in the ring $\mathbb{Z}_{p}$ of $p$-adic integers.

Proof. If $p \mid a$ and $p \mid b$, then $p \nmid c$ since $\operatorname{gcd}(a, b, c)=1$. Hence $f(x) \equiv c \not \equiv 0(\bmod p)$ for any integer $x$. Thus $S\left(f, p^{e}\right)$ is empty in this case. If $p \mid a$ and $p \nmid b$, then there exists a unique integer $x_{0} \in[1, p]$ such that $f\left(x_{0}\right) \equiv b x_{0}+c \equiv 0(\bmod p)$. On the other hand, we have $f^{\prime}\left(x_{0}\right)=2 a x_{0}+b \equiv b \not \equiv 0(\bmod p)$. Then by Hensel's lemma (see, for example, [18]), there is a unique $p$-adic integer $s_{p}$ such that $f\left(s_{p}\right)=0$ and $s_{p} \equiv x_{0}$ $(\bmod p)$. Therefore $\left\langle s_{p}\right\rangle_{p^{e}}$ is the unique solution of $f(x) \equiv 0\left(\bmod p^{e}\right)$ in the interval $\left[1, p^{e}\right]$ satisfying $\left\langle s_{p}\right\rangle_{p^{e}} \equiv s_{p}\left(\bmod p^{e}\right)$. This completes the proof of Lemma 3.1.

Lemma 3.2. Let $a$ be an odd number and let $a^{-1}$ be the inverse of $a$ in the ring $\mathbb{Z}_{2}$ of 2-adic integers. For any positive integer e, each of the following results is true.

(i). If either $e=2\left\lfloor\frac{\nu_{2}(D)}{2}\right\rfloor-1$ with $D_{4} \equiv 2(\bmod 4)$ or $e \leq 2\left\lfloor\frac{\nu_{2}(D)}{2}\right\rfloor-2$, then

$$
S\left(f, 2^{e}\right)=\left\{\left\langle-\frac{a^{-1} b}{2}\right\rangle_{2^{\lceil e / 2\rceil}}+m 2^{\lceil e / 2\rceil}: 0 \leq m<2^{\lfloor e / 2\rfloor}\right\} .
$$

(ii). If either $e=2\left\lfloor\frac{\nu_{2}(D)}{2}\right\rfloor-1$ with $D_{4} \not \equiv 2(\bmod 4)$, or $e=2\left\lfloor\frac{\nu_{2}(D)}{2}\right\rfloor$ with $D_{4} \equiv 1$ $(\bmod 4)$, then

$$
S\left(f, 2^{e}\right)=\left\{\left\langle a^{-1}\left(2^{\frac{\nu_{2}(D)}{2}-1}-\frac{b}{2}\right)\right\rangle_{2^{\frac{\nu_{2}(D)}{2}}}+m 2^{\frac{\nu_{2}(D)}{2}}: 0 \leq m<2^{\lfloor e / 2\rfloor}\right\} .
$$

(iii). If either $e=2\left\lfloor\frac{\nu_{2}(D)}{2}\right\rfloor$ with $D_{4} \not \equiv 1(\bmod 4)$, or $e>2\left\lfloor\frac{\nu_{2}(D)}{2}\right\rfloor$ with $D_{4} \not \equiv 1$ $(\bmod 8)$, then $S\left(f, 2^{e}\right)$ is empty.

(iv). If $D_{4} \equiv 1(\bmod 8)$ and $e>2\left\lfloor\frac{\nu_{2}(D)}{2}\right\rfloor=\nu_{2}(D)$, then

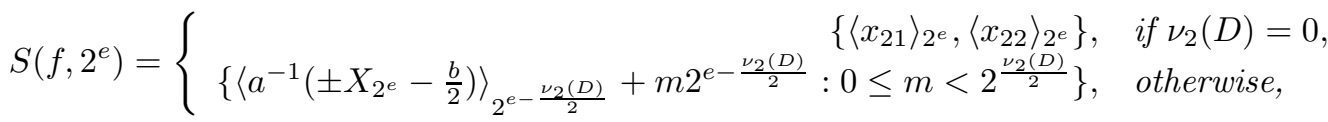

where $x_{21}$ and $x_{22}$ are the only two solutions of $f(x)=0$ in the ring $\mathbb{Z}_{2}$ of 2-adic integers, $X_{2^{e}}$ denotes the smallest root of the congruence $x^{2} \equiv \frac{D}{4}\left(\bmod 2^{e}\right)$ in the interval $\left[1,2^{e-\frac{\nu_{2}(D)}{2}}\right]$.

Proof. First, one can easily deduce from $D=b^{2}-4 a c$ that $\nu_{2}(D)=0$ if $b$ is odd, and $\nu_{2}(D) \geq 2$ if $b$ is even. So for Cases (i) and (ii), since $e \geq 1$, one has $\nu_{2}(D)>0$ and thus $b$ should be even. If $a$ is odd and $b$ is even, then the congruence $f(x) \equiv 0\left(\bmod 2^{e}\right)$ is equivalent to $\left(a x+\frac{b}{2}\right)^{2} \equiv \frac{b^{2}-4 a c}{4} \equiv \frac{D}{4}\left(\bmod 2^{e}\right)$.

(i). Let $e=2\left\lfloor\frac{\nu_{2}(D)}{2}\right\rfloor-1$ with $D_{4} \equiv 2(\bmod 4)$ or $e \leq 2\left\lfloor\frac{\nu_{2}(D)}{2}\right\rfloor-2$. Then $\frac{D}{4} \equiv 0$ $\left(\bmod 2^{e}\right)$. So $y^{2} \equiv \frac{D}{4}\left(\bmod 2^{e}\right)$ has exactly $2^{\lfloor e / 2\rfloor}$ solutions: $m \cdot 2^{\lceil e / 2\rceil}$, where $1 \leq m \leq$ $2^{\lfloor e / 2\rfloor}$. Hence we can derive from $\left(a x+\frac{b}{2}\right)^{2} \equiv \frac{D}{4}\left(\bmod 2^{e}\right)$ that $a x+\frac{b}{2} \equiv m 2^{\lceil e / 2\rceil}$ $\left(\bmod 2^{e}\right)$, which implies that $x \equiv a^{-1}\left(m 2^{\lceil e / 2\rceil}-\frac{b}{2}\right) \equiv-\frac{a^{-1} b}{2}+a^{-1} m 2^{\lceil e / 2\rceil}\left(\bmod 2^{e}\right)$ 
with $0 \leq m<2^{\lfloor e / 2\rfloor}$. Since $a^{-1} m$ runs over a complete residue system modulo $2^{\lfloor e / 2\rfloor}$ as $m$ does, we get $x \equiv\left\langle-\frac{a^{-1} b}{2}\right\rangle_{2^{\lceil e / 2\rceil}}+m 2^{\lceil e / 2\rceil}\left(\bmod 2^{e}\right)$ with $0 \leq m<2^{\lfloor e / 2\rfloor}$. Moreover, for any two integers $m_{1}$ and $m_{2}$ satisfying $0 \leq m_{1} \neq m_{2}<2^{\lfloor e / 2\rfloor}$, we have

$$
\left\langle-\frac{a^{-1} b}{2}\right\rangle_{2^{\lceil e / 2\rceil}}+m_{1} 2^{\lceil e / 2\rceil} \not \equiv\left\langle-\frac{a^{-1} b}{2}\right\rangle_{2^{\lceil e / 2\rceil}}+m_{2} 2^{\lceil e / 2\rceil} \quad\left(\bmod 2^{e}\right) .
$$

So we arrive at the desired result. Thus Part (i) is proved.

(ii). Let $e=2\left\lfloor\frac{\nu_{2}(D)}{2}\right\rfloor-1$ with $D_{4} \not \equiv 2(\bmod 4)$ or $e=2\left\lfloor\frac{\nu_{2}(D)}{2}\right\rfloor$ with $D_{4} \equiv 1$ $(\bmod 4)$. Then $\nu_{2}(D)$ is even. From $\left(a x+\frac{b}{2}\right)^{2} \equiv \frac{D}{4}\left(\bmod 2^{e}\right)$ we deduce that $a x+\frac{b}{2} \equiv$ $(2 m+1) 2^{\frac{\nu_{2}(D)}{2}-1}\left(\bmod 2^{e}\right)$ with $0 \leq m<2^{\lfloor e / 2\rfloor}$. Thus

$$
x \equiv a^{-1}\left((2 m+1) 2^{\frac{\nu_{2}(D)}{2}-1}-\frac{b}{2}\right) \equiv a^{-1}\left(2^{\frac{\nu_{2}(D)}{2}-1}-\frac{b}{2}\right)+a^{-1} m 2^{\frac{\nu_{2}(D)}{2}} \quad\left(\bmod 2^{e}\right)
$$

for $0 \leq m<2^{\lfloor e / 2\rfloor}$. Similarly as in (i), we get $x \equiv\left\langle a^{-1}\left(2^{\frac{\nu_{2}(D)}{2}-1}-\frac{b}{2}\right)\right\rangle_{2^{\frac{\nu_{2}(D)}{2}}}+m 2^{\frac{\nu_{2}(D)}{2}}$ $\left(\bmod 2^{e}\right)$ with $0 \leq m<2^{\lfloor e / 2\rfloor}$. On the other hand,

$\left\langle a^{-1}\left(2^{\frac{\nu_{2}(D)}{2}-1}-\frac{b}{2}\right)\right\rangle_{2} \frac{\nu_{2}(D)}{2}+m_{1} 2^{\frac{\nu_{2}(D)}{2}} \not \equiv\left\langle a^{-1}\left(2^{\frac{\nu_{2}(D)}{2}-1}-\frac{b}{2}\right)\right\rangle_{2} \frac{\nu_{2}(D)}{2}+m_{2} 2^{\frac{\nu_{2}(D)}{2}} \quad\left(\bmod 2^{e}\right)$.

for any two integers $0 \leq m_{1} \neq m_{2}<2^{\lfloor e / 2\rfloor}$. Thus the required result follows. So part (ii) is proved.

(iii). Let $e=2\left\lfloor\frac{\nu_{2}(D)}{2}\right\rfloor$ with $D_{4} \not \equiv 1(\bmod 4)$ or $e>2\left\lfloor\frac{\nu_{2}(D)}{2}\right\rfloor$ with $D_{4} \not \equiv 1(\bmod 8)$. If $\nu_{2}(D)=0$, then we can derive from $D_{4}=D=b^{2}-4 a c \not \equiv 1(\bmod 8)$ that $b$ and $c$ are both odd numbers. Thus for any positive integer $n, f(n) \not \equiv 0(\bmod 2)$. This infers that $S\left(f, 2^{e}\right)$ is empty.

If $\nu_{2}(D) \geq 2$, since $e=2\left\lfloor\frac{\nu_{2}(D)}{2}\right\rfloor$ with $D_{4} \not \equiv 1(\bmod 4)$ or $e>2\left\lfloor\frac{\nu_{2}(D)}{2}\right\rfloor$ with $D_{4} \not \equiv 1$ $(\bmod 8)$, then $y^{2} \equiv D_{4}\left(\bmod 2^{e-2\left\lfloor\frac{\nu_{2}(D)}{2}\right\rfloor+2}\right)$ has no solution. Hence there is no integer $y$ satisfying $y^{2} \equiv \frac{D}{4}\left(\bmod 2^{e}\right)$. Thus $\left(a x+\frac{b}{2}\right)^{2} \equiv \frac{D}{4}\left(\bmod 2^{e}\right)$ has no solution, which means that $S\left(f, 2^{e}\right)$ is empty. This concludes part (iii).

(iv). Let $e>2\left\lfloor\frac{\nu_{2}(D)}{2}\right\rfloor$ and $D_{4} \equiv 1(\bmod 8)$. If $\nu_{2}(D)=0$, then it follows from $D_{4} \equiv 1$ $(\bmod 8)$ that $b$ is odd and $c$ is even. Thus one has that $f(0) \equiv 0(\bmod 2)$ and $f(1) \equiv 0$ $(\bmod 2)$. On the other hand, $f^{\prime}(0) \equiv f^{\prime}(1) \equiv b \not \equiv 0(\bmod 2)$. So by Hensel's lemma, there are exactly two 2 -adic integers $x_{21}$ and $x_{22}$ such that $x_{21} \equiv 0(\bmod 2), x_{22} \equiv 1$ $(\bmod 2)$ and $f\left(x_{21}\right)=f\left(x_{22}\right)=0$. Thus $\left\langle x_{21}\right\rangle_{2^{e}}$ and $\left\langle x_{22}\right\rangle_{2^{e}}$ are exactly two solutions of the congruence $f(x) \equiv 0\left(\bmod 2^{e}\right)$ in the interval $\left[1,2^{e}\right]$.

If $\nu_{2}(D) \geq 2$, then $b$ is even. By the definition of $D_{4}$, we know that $\nu_{2}(D)$ is even if $D_{4} \equiv 1(\bmod 8)$. Since $D_{4} \equiv 1(\bmod 8)$ and $e>2\left\lfloor\frac{\nu_{2}(D)}{2}\right\rfloor=\nu_{2}(D)$, it is known (see Theorem 5.1 of page 44 in [16]) that $y^{2} \equiv D_{4}\left(\bmod 2^{e+2-\nu_{2}(D)}\right)$ has just four solutions in the interval $\left[1,2^{e+2-\nu_{2}(D)}\right)$. Let $y_{1}$ denote the smallest solution in the interval $\left[1,2^{e+2-\nu_{2}(D)}\right)$ of $y^{2} \equiv D_{4}\left(\bmod 2^{e+2-\nu_{2}(D)}\right)$. Evidently, $y_{1}$ is odd and $y_{1} \in\left[1,2^{e-\nu_{2}(D)}\right)$. Then the four solutions of $y^{2} \equiv D_{4}\left(\bmod 2^{e+2-\nu_{2}(D)}\right)$ are as follows: $y_{1}, 2^{e+1-\nu_{2}(D)}-$ $y_{1}, y_{1}+2^{e+1-\nu_{2}(D)}, 2^{e+2-\nu_{2}(D)}-y_{1}$. Thus the congruence $y^{2} \equiv \frac{D}{4} \equiv 2^{\nu_{2}(D)-2} D_{4}\left(\bmod 2^{e}\right)$ has the following solutions: $y=2^{\frac{\nu_{2}(D)}{2}-1}\left(y_{1}+m_{1} 2^{e-\nu_{2}(D)+1}\right)=2^{\frac{\nu_{2}(D)}{2}-1} y_{1}+m_{1} 2^{e-\frac{\nu_{2}(D)}{2}}$ or $y=2^{\frac{\nu_{2}(D)}{2}-1}\left(2^{e-\nu_{2}(D)+1}-y_{1}+m_{2} 2^{e-\nu_{2}(D)+1}\right)=2^{e-\frac{\nu_{2}(D)}{2}}-2^{\frac{\nu_{2}(D)}{2}-1} y_{1}+m_{2} 2^{e-\frac{\nu_{2}(D)}{2}}$, where $0 \leq m_{1}, m_{2}<2^{\frac{\nu_{2}(D)}{2}}$ are integers. Now let $X_{2^{e}}=2^{\frac{\nu_{2}(D)}{2}-1} y_{1}$. Then from $\left(a x+\frac{b}{2}\right)^{2} \equiv \frac{D}{4} \equiv 2^{\nu_{2}(D)-2} D_{4}\left(\bmod 2^{e}\right)$ we get that $a x+\frac{b}{2} \equiv \pm X_{2^{e}}+m 2^{e-\frac{\nu_{2}(D)}{2}}\left(\bmod 2^{e}\right)$, 
which implies that $x \equiv\left\langle a^{-1}\left( \pm X_{2^{e}}-\frac{b}{2}\right)\right\rangle_{2^{e-\frac{\nu_{2}(D)}{2}}}+a^{-1} m 2^{e-\frac{\nu_{2}(D)}{2}}\left(\bmod 2^{e}\right)$ for any integer $m$ with $0 \leq m<2^{\frac{\nu_{2}(D)}{2}}$. Since $a^{-1} m$ runs over a complete residue system modulo $2^{\frac{\nu_{2}(D)}{2}}$ as $m$ does so, we obtain that $x \equiv\left\langle a^{-1}\left( \pm X_{2^{e}}-\frac{b}{2}\right)\right\rangle_{2^{e-\frac{\nu_{2}(D)}{2}}}+m 2^{e-\frac{\nu_{2}(D)}{2}}\left(\bmod 2^{e}\right)$ for any integer $m$ with $0 \leq m<2^{\frac{\nu_{2}(D)}{2}}$. One can easily check that all the $2^{\frac{\nu_{2}(D)}{2}+1}$ elements of the set $\left\{\left\langle a^{-1}\left( \pm X_{2^{e}}-\frac{b}{2}\right)\right\rangle_{2^{e-\frac{\nu_{2}(D)}{2}}}+m 2^{e-\frac{\nu_{2}(D)}{2}}: 0 \leq m<2^{\frac{\nu_{2}(D)}{2}}\right\}$ are pairwise incongruent modulo $2^{e}$. Thus the desired result follows immediately. The proof of Lemma 3.2 is complete.

Lemma 3.3. Let $p$ be an odd prime with $p \nmid a$, and let $D_{p}$ be defined as in (1.3). By $(2 a)^{-1}$ we denote the inverse of $2 a$ in the ring $\mathbb{Z}_{p}$ of $p$-adic integers. For any positive integer $e$, each of the following results is true.

(i). If $e \leq \nu_{p}(D)$, then $S\left(f, p^{e}\right)=\left\{\left\langle-(2 a)^{-1} b\right\rangle_{p^{\lceil e / 2\rceil}}+m p^{\lceil e / 2\rceil}: 0 \leq m<p^{\lfloor e / 2\rfloor}\right\}$.

(ii). If e $>\nu_{p}(D)$ with either $\nu_{p}(D)$ being odd or $\left(\frac{D_{p}}{p}\right)=-1$, then $S\left(f, p^{e}\right)$ is empty.

(iii). If $e>\nu_{p}(D)$ with $\nu_{p}(D)$ being even and $\left(\frac{D_{p}}{p}\right)=1$, then

$$
S\left(f, p^{e}\right)=\left\{\left\langle(2 a)^{-1}\left( \pm X_{p^{e}}-b\right)\right\rangle_{p^{e-\frac{\nu_{p}(D)}{2}}}+m p^{e-\frac{\nu_{p}(D)}{2}}: 0 \leq m<p^{\frac{\nu_{p}(D)}{2}}\right\},
$$

where $X_{p^{e}}$ is the smallest solution of $x^{2} \equiv D\left(\bmod p^{e}\right)$ in the interval $\left[1, p^{e-\frac{\nu_{p}(D)}{2}}\right]$.

Proof. Since $p \nmid 2 a, f(x) \equiv 0\left(\bmod p^{e}\right)$ is equivalent to $(2 a x+b)^{2} \equiv D\left(\bmod p^{e}\right)$.

(i). Let $e \leq \nu_{p}(D)$. Then one has $2 a x+b \equiv m p^{\lceil e / 2\rceil}\left(\bmod p^{e}\right)$ for some integers $1 \leq$ $m \leq p^{\lfloor e / 2\rfloor}$. Hence $x \equiv(2 a)^{-1}\left(m p^{\lceil e / 2\rceil}-b\right)\left(\bmod p^{e}\right)$ for $1 \leq m \leq p^{\lfloor e / 2\rfloor}$. Moreover, we have $(2 a)^{-1}\left(m_{1} p^{\lceil e / 2\rceil}-b\right) \not \equiv(2 a)^{-1}\left(m_{2} p^{\lceil e / 2\rceil}-b\right)\left(\bmod p^{e}\right)$ for any two integers $m_{1}$ and $m_{2}$ with $1 \leq m_{1} \neq m_{2} \leq p^{\lfloor e / 2\rfloor}$. On the other hand, since $(2 a)^{-1} m$ runs over a complete residue system modulo $p^{\lfloor e / 2\rfloor}$ as $m$ does, we get that $x \equiv\left\langle-(2 a)^{-1} b\right\rangle_{p^{\lceil e / 2\rceil}}+m p^{\lceil e / 2\rceil}$ $\left(\bmod p^{e}\right)$ with $0 \leq m<p^{\lfloor e / 2\rfloor}$. Thus we derive the required result immediately.

(ii). Let $e>\nu_{p}(D)$ with either $\nu_{p}(D)$ odd or $\left(\frac{D_{p}}{p}\right)=-1$. Suppose that there is an integer $n_{0}$ such that $n_{0}^{2} \equiv D\left(\bmod p^{e}\right)$. Then $n_{0}^{2} \equiv p^{\nu_{p}(D)} D_{p}\left(\bmod p^{e}\right)$. Since $e>\nu_{p}(D)$, $p^{\left\lceil\frac{\nu_{p}(D)}{2}\right\rceil}$ divides $n_{0}$. If $\nu_{p}(D)$ is odd, then $n_{0}^{2} \equiv p^{\nu_{p}(D)+1} \frac{n_{0}^{2}}{p^{\nu_{p}(D)+1}} \equiv p^{\nu_{p}(D)} D_{p}\left(\bmod p^{e}\right)$. Hence $p \cdot \frac{n_{0}^{2}}{p^{\nu p(D)+1}} \equiv D_{p}\left(\bmod p^{e-\nu_{p}(D)}\right)$, which is a contradiction. If $\nu_{p}(D)$ is even and $\left(\frac{D_{p}}{p}\right)=-1$, then $\left(\frac{n_{0}}{p^{\nu_{p}(D) / 2}}\right)^{2} \equiv D_{p}\left(\bmod p^{e-\nu_{p}(D)}\right)$, which is impossible since $\left(\frac{D_{p}}{p}\right)=-1$. Thus there is no integer $y$ such that $y^{2} \equiv D\left(\bmod p^{e}\right)$. It follows immediately that the congruence $(2 a x+b)^{2} \equiv D\left(\bmod p^{e}\right)$ has no solution and $S\left(f, p^{e}\right)$ is empty.

(iii). Let $e>\nu_{p}(D)$ with $\nu_{p}(D)$ even and $\left(\frac{D_{p}}{p}\right)=1$. Then by Hensel's lemma, the congruence $y^{2} \equiv D_{p}\left(\bmod p^{e-\nu_{p}(D)}\right)$ has exactly two solutions $y_{0}$ and $p^{e-\nu_{p}(D)}-$ $y_{0}$ in the interval $\left[1, p^{e-\nu_{p}(D)}\right]$. Thus for all integers $0 \leq m<p^{\frac{\nu_{p}(D)}{2}}$, we have $y=$ $p^{\frac{\nu_{p}(D)}{2}}\left(y_{0}+m p^{e-\nu_{p}(D)}\right)=p^{\frac{\nu_{p}(D)}{2}} y_{0}+m p^{e-\frac{\nu_{p}(D)}{2}}$ and $y=p^{e-\frac{\nu_{p}(D)}{2}}-p^{\frac{\nu_{p}(D)}{2}} y_{0}+m p^{e-\frac{\nu_{p}(D)}{2}}$, are solutions of the congruence $y^{2} \equiv D\left(\bmod p^{e}\right)$. Let now $X_{p^{e}}=p^{\frac{\nu_{p}(D)}{2}} y_{0}$. Then we derive that $2 a x+b \equiv\left\langle \pm X_{p^{e}}\right\rangle_{p^{e-\frac{\nu_{p}(D)}{2}}}+m p^{e-\frac{\nu_{p}(D)}{2}}\left(\bmod p^{e}\right)$. Since $(2 a)^{-1} m$ runs over the complete residue system as $m$ does, we get $x \equiv\left\langle(2 a)^{-1}\left( \pm X_{p^{e}}-b\right)\right\rangle_{p^{e-\frac{\nu_{p}(D)}{2}}}+m p^{e-\frac{\nu_{p}(D)}{2}}$ $\left(\bmod p^{e}\right)$ where $0 \leq m<p^{\frac{\nu_{p}(D)}{2}}$. Obviously, any two classes of the above $2 p^{\frac{\nu_{p}(D)}{2}}$ 
residue classes are distinct modulo $p^{e}$. This concludes the desired result. Lemma 3.3 is proved.

Once we determine the set of solutions of $f(x) \equiv 0\left(\bmod p^{e}\right)$, we naturally want to know more information about these solutions. First, we introduce the following concepts, which are important ingredients in the process of determining the local periods.

Definition 3.4. Let $e$ be a nonnegative integer. If $S\left(f, p^{e}\right)$ is nonempty, then for any $x_{1}, x_{2} \in S\left(f, p^{e}\right)$, we define the distance of $x_{1}$ and $x_{2}$ by

$$
d_{p^{e}}\left(x_{1}, x_{2}\right):=\min \left\{\left\langle x_{1}-x_{2}\right\rangle_{p^{e}},\left\langle x_{2}-x_{1}\right\rangle_{p^{e}}\right\} .
$$

Clearly, for any $x_{1}, x_{2} \in S\left(f, p^{e}\right), d_{p^{e}}\left(x_{1}, x_{2}\right)$ equals $\min \left\{\left|x_{1}-x_{2}\right|, p^{e}-\left|x_{1}-x_{2}\right|\right\}$ if $x_{1} \neq x_{2}$, and is $p^{e}$ if $x_{1}=x_{2}$.

Definition 3.5. Let $e$ be a nonnegative integer. We define the minimal distance, denoted by $d_{p^{e}}$, among the solutions of $f(x) \equiv 0\left(\bmod p^{e}\right)$ as follows: $d_{p^{0}}:=1$, and for $e \geq 1$,

$$
d_{p^{e}}:=\left\{\begin{aligned}
\min \left\{d_{p^{e}}\left(x_{i}, x_{j}\right): x_{i}, x_{j} \in S\left(f, p^{e}\right)\right\}, & \text { if } S\left(f, p^{e}\right) \text { is nonempty } \\
\infty, & \text { if } S\left(f, p^{e}\right) \text { is empty. }
\end{aligned}\right.
$$

In what follows, we study the arithmetic properties of the minimal distance $d_{p^{e}}$.

Lemma 3.6. Let $S\left(f, p^{e}\right)$ be nonempty. Then there exists a positive integer $n$ such that $\nu_{p}(f(n)) \geq e$ and $\nu_{p}\left(f\left(n+d_{p^{e}}\right)\right) \geq e$. Further, if $d_{p^{e}}<d_{p^{e+1}}$, then there is a positive integer $m$ such that $\nu_{p}(f(m))=e$.

Proof. First let $\left|S\left(f, p^{e}\right)\right|=1$. Then $S\left(f, p^{e}\right)$ contains only one element, saying $x_{0}$, and $d_{p^{e}}=p^{e}$. Thus one can pick $n=x_{0}$ to arrive at the desired result. Lemma 3.6 is true in this case. Now let $\left|S\left(f, p^{e}\right)\right| \geq 2$. Then by Definitions 3.5 and 3.4, there are $x_{1}, x_{2} \in S\left(f, p^{e}\right)$ with $x_{1}<x_{2}$ such that $d_{p^{e}}=d_{p^{e}}\left(x_{1}, x_{2}\right)$. It follows that

$$
d_{p^{e}}=\min \left(x_{2}-x_{1}, p^{e}+x_{1}-x_{2}\right) .
$$

If $x_{2}-x_{1} \leq \frac{1}{2} p^{e}$, by (3.1) we have $d_{p^{e}}=x_{2}-x_{1}$. Take $n=x_{1}$. Then we have $\nu_{p}(f(n))=\nu_{p}\left(f\left(x_{1}\right)\right) \geq e$ and $\nu_{p}\left(f\left(n+d_{p^{e}}\right)\right)=\nu_{p}\left(f\left(x_{2}\right)\right) \geq e$ as required.

If $x_{2}-x_{1}>\frac{1}{2} p^{e}$, then by (3.1) we have $d_{p^{e}}=p^{e}+x_{1}-x_{2}$. Hence taking $n=x_{2}$ gives that $n+d_{p^{e}}=x_{1}+p^{e}$. But $f\left(x_{1}+p^{e}\right) \equiv f\left(x_{1}\right)\left(\bmod p^{e}\right)$ and $f\left(x_{1}\right) \equiv 0\left(\bmod p^{e}\right)$. So we have $f\left(n+d_{p^{e}}\right) \equiv 0\left(\bmod p^{e}\right)$. The first part of Lemma 3.6 is proved.

Now suppose that $d_{p^{e}}<d_{p^{e+1}}$. Since $S\left(f, p^{e}\right)$ is nonempty, by the first part we can find a positive integer $n_{1}$ such that $\nu_{p}\left(f\left(n_{1}\right)\right) \geq e$ and $\nu_{p}\left(f\left(n_{1}+d_{p^{e}}\right)\right) \geq e$. Suppose that $\nu_{p}\left(f\left(n_{1}\right)\right)>e$ and $\nu_{p}\left(f\left(n_{1}+d_{p^{e}}\right)\right)>e$. Then $\nu_{p}\left(f\left(n_{1}\right)\right) \geq e+1$ and $\nu_{p}\left(f\left(n_{1}+d_{p^{e}}\right)\right) \geq e+1$. Thus $\nu_{p}\left(f\left(\left\langle n_{1}\right\rangle_{p^{e+1}}\right)\right) \geq e+1$ and $\nu_{p}\left(f\left(\left\langle n_{1}+d_{p^{e}}\right\rangle_{p^{e+1}}\right)\right) \geq e+1$. That is, $\left\langle n_{1}\right\rangle_{p^{e+1}}$ and $\left\langle n_{1}+d_{p^{e}}\right\rangle_{p^{e+1}}$ are belonging to the set $S\left(f, p^{e+1}\right)$. Since $\left\langle n_{1}+d_{p^{e}}\right\rangle_{p^{e+1}}-\left\langle n_{1}\right\rangle_{p^{e+1}} \equiv$ $d_{p^{e}}\left(\bmod p^{e+1}\right)$, we get that $\left|\left\langle n_{1}+d_{p^{e}}\right\rangle_{p^{e+1}}-\left\langle n_{1}\right\rangle_{p^{e+1}}\right|=d_{p^{e}}$ or $p^{e+1}-d_{p^{e}}$. It then follows that $d_{p^{e+1}} \leq d_{p^{e+1}}\left(\left\langle n_{1}\right\rangle_{p^{e+1}},\left\langle n_{1}+d_{p^{e}}\right\rangle_{p^{e+1}}\right)=\min \left(d_{p^{e}}, p^{e+1}-d_{p^{e}}\right) \leq d_{p^{e}}$ which contradicts with the assumption $d_{p^{e}}<d_{p^{e+1}}$. Therefore we have either $\nu_{p}\left(f\left(n_{1}\right)\right)=e$ or $\nu_{p}\left(f\left(n_{1}+d_{p^{e}}\right)\right)=e$. This concludes Lemma 3.6.

Lemma 3.7. For any given prime number $p$, the sequence $\left\{d_{p^{e}}\right\}_{e=0}^{\infty}$ is nondecreasing.

Proof. Evidently, it is enough to prove $d_{p^{e+1}} \geq d_{p^{e}}$ for any nonnegative integer $e$. Now let $e$ be any given nonnegative integer. If $S\left(f, p^{e+1}\right)$ is empty, then $d_{p^{e+1}}=\infty$ and so $d_{p^{e+1}} \geq d_{p^{e}}$ as desired. In what follows we assume that $S\left(f, p^{e+1}\right)$ is not empty.

Clearly, it suffices to prove that $d_{p^{e+1}}\left(x_{1}, x_{2}\right) \geq d_{p^{e}}$ for any two elements $x_{1}, x_{2} \in$ $S\left(f, p^{e+1}\right)$, from which we derive that $d_{p^{e+1}} \geq d_{p^{e}}$. Taking any two elements $x_{1}, x_{2} \in$ 
$S\left(f, p^{e+1}\right)$, then we have that $x_{1}=y_{1}+t_{1} p^{e}$ and $x_{2}=y_{2}+t_{2} p^{e}$ for some integers $t_{1}, t_{2}, y_{1}, y_{2}$ with $0 \leq t_{1}, t_{2} \leq p-1$ and $y_{1}, y_{2} \in S\left(f, p^{e}\right)$. It is clear that

$$
d_{p^{e+1}}\left(x_{1}, x_{2}\right)=\min \left\{\left\langle\left(t_{1}-t_{2}\right) p^{e}\right\rangle_{p^{e+1}},\left\langle\left(t_{2}-t_{1}\right) p^{e}\right\rangle_{p^{e+1}}\right\} \geq p^{e}=d_{p^{e}}\left(y_{1}, y_{2}\right) \geq d_{p^{e}}
$$

if $y_{1}=y_{2}$. Let now $y_{1} \neq y_{2}$. Then $x_{1} \neq x_{2}$. We have $d_{p^{e}}\left(y_{1}, y_{2}\right)=\min \left\{\left|y_{1}-y_{2}\right|, p^{e}-\right.$ $\left.\left|y_{1}-y_{2}\right|\right\}$ and $d_{p^{e+1}}\left(x_{1}, x_{2}\right)=\min \left\{\left|y_{1}-y_{2}+\left(t_{1}-t_{2}\right) p^{e}\right|, p^{e+1}-\left|y_{1}-y_{2}+\left(t_{1}-t_{2}\right) p^{e}\right|\right\}$.

If $t_{1}=t_{2}$, then $d_{p^{e+1}}\left(x_{1}, x_{2}\right)=\left|y_{1}-y_{2}\right| \geq d_{p^{e}}\left(y_{1}, y_{2}\right) \geq d_{p^{e}}$. If $t_{1} \neq t_{2}$, then

$$
\left|y_{1}-y_{2}+\left(t_{1}-t_{2}\right) p^{e}\right| \geq\left|\left(t_{1}-t_{2}\right) p^{e}\right|-\left|y_{1}-y_{2}\right| \geq p^{e}-\left|y_{1}-y_{2}\right| \geq d_{p^{e}}\left(y_{1}, y_{2}\right) \geq d_{p^{e}}
$$

and

$$
\begin{aligned}
p^{e+1}-\left|y_{1}-y_{2}+\left(t_{1}-t_{2}\right) p^{e}\right| & \geq p^{e+1}-\left|y_{1}-y_{2}\right|-\left|\left(t_{1}-t_{2}\right) p^{e}\right| \\
& \geq p^{e}-\left|y_{1}-y_{2}\right| \geq d_{p^{e}}\left(y_{1}, y_{2}\right) \geq d_{p^{e}} .
\end{aligned}
$$

It follows that $d_{p^{e+1}}\left(x_{1}, x_{2}\right) \geq d_{p^{e}}$. So Lemma 3.7 is proved.

Lemma 3.8. If $p \mid a$, then for any positive integer $e$, we have $d_{p^{e}}= \begin{cases}\infty, & \text { if } p \mid b, \\ p^{e}, & \text { if } p \nmid b .\end{cases}$

Proof. It follows immediately from Lemma 3.1 that Lemma 3.8 is true.

Lemma 3.9. Let a be odd and e be a positive integer.

(i). If $e=\nu_{2}(D)$ with $D_{4} \equiv 1(\bmod 4)$ or $e \leq 2\left\lfloor\frac{\nu_{2}(D)}{2}\right\rfloor-1$, then $d_{2^{e}}=2^{\lceil e / 2\rceil}$. Also $d_{2^{e}}$ equals the smallest positive root of $a^{2} x^{2}-D \equiv 0\left(\bmod 2^{e}\right)$. Moreover, the distance between any two distinct solutions of $f(x) \equiv 0\left(\bmod 2^{e}\right)$ is divisible by $2^{\lceil e / 2\rceil}$ if $e \geq 2$.

(ii). If $e=2\left\lfloor\frac{\nu_{2}(D)}{2}\right\rfloor$ with $D_{4} \not \equiv 1(\bmod 4)$ or $e>2\left\lfloor\frac{\nu_{2}(D)}{2}\right\rfloor$ with $D_{4} \not \equiv 1(\bmod 8)$, then $d_{2^{e}}=\infty$.

(iii). If $e>2\left\lfloor\frac{\nu_{2}(D)}{2}\right\rfloor=\nu_{2}(D)$ with $D_{4} \equiv 1(\bmod 8)$, then $d_{2^{e}}$ is equal to the smallest positive root of the congruence $a^{2} x^{2}-D \equiv 0\left(\bmod 2^{e+1}\right)$.

Proof. (i). It is easy to check that $d_{2^{e}}=2^{e}=2^{\lceil e / 2\rceil}$ if $e=1$. So it is enough to prove part (i) for the case $e \geq 2$. In what follows we let $e \geq 2$.

If $e=2\left\lfloor\frac{\nu_{2}(D)}{2}\right\rfloor-1$ with $D_{4} \equiv 2(\bmod 4)$ or $e \leq 2\left\lfloor\frac{\nu_{2}(D)}{2}\right\rfloor-2$, then by Lemma 3.2 (i), $\left\langle-\frac{a^{-1} b}{2}\right\rangle_{2^{\lceil e / 2\rceil}}+i 2^{\lceil e / 2\rceil}$ and $\left\langle-\frac{a^{-1} b}{2}\right\rangle_{2^{\lceil e / 2\rceil}}+2^{\lceil e / 2\rceil}$ are two roots of $f(x) \equiv 0\left(\bmod 2^{e}\right)$, where $0 \leq i \neq j<2^{\lfloor e / 2\rfloor}$. It is easy to see that

$$
\left\langle-\frac{a^{-1} b}{2}\right\rangle_{2^{\lceil e / 2\rceil}}+i 2^{\lceil e / 2\rceil}-\left(\left\langle-\frac{a^{-1} b}{2}\right\rangle_{2^{\lceil e / 2\rceil}}+j 2^{\lceil e / 2\rceil}\right) \equiv(i-j) 2^{\lceil e / 2\rceil} \quad\left(\bmod 2^{e}\right) .
$$

We can find two integers $i_{0}$ and $j_{0}$ with $0 \leq i_{0} \neq j_{0}<2^{\lfloor e / 2\rfloor}$ such that $\left(i_{0}-j_{0}\right)=1$. Then by (3.2), we have $d_{2^{e}}=2^{\lceil e / 2\rceil}$ as required.

If either $e=2\left\lfloor\frac{\nu_{2}(D)}{2}\right\rfloor-1$ with $D_{4} \not \equiv 2(\bmod 4)$ or $e=2\left\lfloor\frac{\nu_{2}(D)}{2}\right\rfloor=\nu_{2}(D)$ with $D_{4} \equiv 1(\bmod 4)$, then $\nu_{2}(D) \geq 2$ is even in this case. For any two integers $i$ and $j$ with $0 \leq i \neq j<2^{\frac{\nu_{2}(D)}{2}}$, we have

$$
\left\langle a^{-1}\left(2^{\frac{\nu_{2}(D)}{2}-1}-\frac{b}{2}\right)\right\rangle_{2} \frac{\nu_{2}(D)}{2}+i 2^{\frac{\nu_{2}(D)}{2}}-\left\langle a^{-1}\left(2^{\frac{\nu_{2}(D)}{2}-1}-\frac{b}{2}\right)\right\rangle_{2^{\frac{\nu_{2}(D)}{2}}}-j 2^{\frac{\nu_{2}(D)}{2}}=(i-j) 2^{\frac{\nu_{2}(D)}{2}} \text {. }
$$

Then by Lemma 3.2 (ii), we have $d_{2^{e}}=2^{\frac{\nu_{2}(D)}{2}}=2^{\lceil e / 2\rceil}$ as desired. Evidently, under the assumptions of part (i), we have $e \leq \nu_{2}(D)$. So $2^{\lceil e / 2\rceil}$ is the smallest positive root of $a^{2} x^{2}-D \equiv 0\left(\bmod 2^{e}\right)$. Finally, by (3.2) and $(3.3), 2^{\lceil e / 2\rceil}$ divides the distance between any two distinct solutions of $f(x) \equiv 0\left(\bmod 2^{e}\right)$. 
(ii). By Lemma 3.2 (iii), $S\left(f, 2^{e}\right)$ is empty in this case, which means that $d_{2^{e}}=\infty$.

(iii). Since $D_{4} \equiv 1(\bmod 8), \nu_{2}(D)$ is even. If $\nu_{2}(D)=0$, then $D=D_{4}$. Clearly $d_{2}=1$ is the smallest positive root of $a^{2} x^{2}-D \equiv 0\left(\bmod 2^{2}\right)$. In what follows, we only need to consider the case either $\nu_{2}(D)=0$ and $e \geq 2$, or $\nu_{2}(D) \geq 2$ and $e \geq \nu_{2}(D)+1$.

For any integer $x_{1}$ satisfying $f\left(x_{1}\right) \equiv 0\left(\bmod 2^{e}\right)$, we have $\left(2 a x_{1}+b\right)^{2} \equiv D\left(\bmod 2^{e+2}\right)$. Hence $\left(2 a x_{1}+b\right)^{2} \equiv D\left(\bmod 2^{e+1}\right)$. Note that the discriminant of $x^{2}-D$ equals $4 D$. Since $\nu_{2}(4 D)=\nu_{2}(D)+2$ and $\frac{4 D}{2^{\nu_{2}(D)+2}}=D_{4} \equiv 1(\bmod 8)$, then by Lemma 3.2 (iv), we obtain the exactly $2^{\frac{\nu_{2}(D)+2}{2}+1}=2^{\frac{\nu_{2}(D)}{2}+2}$ roots of $x^{2}-D \equiv 0\left(\bmod 2^{e+1}\right)$. Now let $y_{e+1}$ be the smallest positive root of $a^{2} x^{2}-D \equiv 0\left(\bmod 2^{e+1}\right)$. Then $y_{e+1} \in\left[1,2^{e-1-\nu_{2}(D) / 2}\right)$ and $2^{\frac{\nu_{2}(D)}{2}}$ divides $y_{e+1}$. We claim that the set of solutions of $x^{2}-D \equiv 0\left(\bmod 2^{e+1}\right)$ in the interval $\left[1,2^{e+1}\right]$ is given as follows:

$$
\left\{\left\langle \pm a y_{e+1}\right\rangle_{2^{e-\frac{\nu_{2}(D)}{2}}}+m 2^{e-\frac{\nu_{2}(D)}{2}}: 0 \leq m<2^{\frac{\nu_{2}(D)}{2}+1}\right\} .
$$

On the one hand, since $2^{\frac{\nu_{2}(D)}{2}}$ divides $y_{e+1}$ and $e>\nu_{2}(D)$, one can easily check that each one in the set (3.4) satisfies $x^{2}-D \equiv 0\left(\bmod 2^{e+1}\right)$. On the other hand, it is clear that any two elements in the set (3.4) are incongruent modulo $2^{e+1}$ and the set (3.4) holds $2^{\frac{\nu_{2}(D)}{2}+2}$ elements. Thus (3.4) gives all the solutions of the congruence $x^{2}-D \equiv 0$ $\left(\bmod 2^{e+1}\right)$ in the interval $\left[1,2^{e+1}\right]$. The claim is proved.

Now from the claim we get that $2 a x_{1}+b \equiv \pm a y_{e+1}+m_{0} 2^{e-\frac{\nu_{2}(D)}{2}}\left(\bmod 2^{e+1}\right)$ for some $0 \leq m_{0}<2^{\frac{\nu_{2}(D)}{2}+1}$, which implies that $2 a x_{1}+b \equiv a y_{e+1}$ or $-a y_{e+1}\left(\bmod 2^{e-\frac{\nu_{2}(D)}{2}}\right)$.

In what follows we show that $d_{2^{e}}=y_{e+1}$. Since the proof for the case $2 a x_{1}+b \equiv$ $-a y_{e+1}\left(\bmod 2^{e-\frac{\nu_{2}(D)}{2}}\right)$ is similar as that of the case $2 a x_{1}+b \equiv a y_{e+1}\left(\bmod 2^{e-\frac{\nu_{2}(D)}{2}}\right)$, we only treat with the latter case. Let $2 a x_{1}+b \equiv a y_{e+1}\left(\bmod 2^{e-\frac{\nu_{2}(D)}{2}}\right)$. Then we have

$$
\begin{gathered}
a\left(x_{1}-y_{e+1}\right)^{2}+b\left(x_{1}-y_{e+1}\right)+c \equiv-\left(2 a x_{1}+b\right) y_{e+1}+a y_{e+1}^{2} \\
\equiv-\left(a y_{e+1}+t 2^{e-\frac{\nu_{2}(D)}{2}}\right) y_{e+1}+a y_{e+1}^{2} \equiv-t y_{e+1} 2^{e-\frac{\nu_{2}(D)}{2}} \quad\left(\bmod 2^{e}\right)
\end{gathered}
$$

for some integer $t$. Since $2^{\frac{\nu_{2}(D)}{2}} \mid y_{e+1}$, we then derive that $f\left(x_{1}-y_{e+1}\right) \equiv-t y_{e+1} 2^{e-\frac{\nu_{2}(D)}{2}} \equiv$ $0\left(\bmod 2^{e}\right)$. So $x_{1}-y_{e+1}$ is a solution of $f(x) \equiv 0\left(\bmod 2^{e}\right)$. But $x_{1}$ is a solution of $f(x) \equiv 0\left(\bmod 2^{e}\right)$. It follows that $d_{2^{e}} \leq d_{2^{e}}\left(x_{1}, x_{1}-y_{e+1}\right) \leq y_{e+1}$.

Noticing that $f\left(x_{1}+m 2^{e-\frac{\nu_{2}(D)}{2}}\right)=f\left(x_{1}\right)+\left(2 a x_{1}+b\right) m 2^{e-\frac{\nu_{2}(D)}{2}}+a m^{2} 2^{2 e-\nu_{2}(D)}$, $2^{\frac{\nu_{2}(D)}{2}} \mid\left(2 a x_{1}+b\right)$ and $e>\nu_{2}(D)$, we deduce that $f\left(x_{1}+m 2^{e-\frac{\nu_{2}(D)}{2}}\right) \equiv 0\left(\bmod 2^{e}\right)$ for any integer $m$. Replacing $x_{1}$ by $x_{1}-y_{e+1}$ gives that $f\left(x_{1}-y_{e+1}+m 2^{e-\frac{\nu_{2}(D)}{2}}\right) \equiv 0\left(\bmod 2^{e}\right)$ for any integer $m$. Thus by Lemma 3.2 (iv), one knows that $\left\langle x_{1}\right\rangle_{2^{e-\frac{\nu_{2}(D)}{2}}},\left\langle x_{1}-y_{e+1}\right\rangle_{2^{e-\frac{\nu_{2}(D)}{2}}}$ are the only two distinct solutions of $f(x) \equiv 0\left(\bmod 2^{e}\right)$ in the interval $\left[1,2^{e-\frac{\nu_{2}(D)}{2}}\right]$.

Since $\left\langle x_{1}\right\rangle_{2^{e-\frac{\nu_{2}(D)}{2}}}-\left\langle x_{1}-y_{e+1}\right\rangle_{2^{e-\frac{\nu_{2}(D)}{2}}} \equiv y_{e+1}\left(\bmod 2^{e-\frac{\nu_{2}(D)}{2}}\right)$, one can easily check that $\left\langle x_{1}\right\rangle_{2^{e-\frac{\nu_{2}(D)}{2}}}-\left\langle x_{1}-y_{e+1}\right\rangle_{2^{e-\frac{\nu_{2}(D)}{2}}}$ is a solution of $a^{2} x^{2}-D \equiv 0\left(\bmod 2^{e+1}\right)$. It then follows immediately that so is

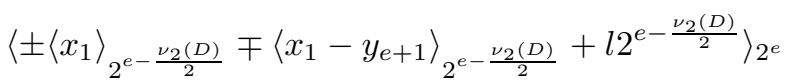

for any integer $l$. However, by the definition of $d_{2^{e}}$ and Lemma 3.2 (iv), $d_{2^{e}}$ must be of the form (3.5) for some integer $l$. Therefore $d_{2^{e}}$ is a solution of $a^{2} x^{2}-D \equiv 0\left(\bmod 2^{e+1}\right)$. Then by the minimality of $y_{e+1}$, we have $d_{2^{e}} \geq y_{e+1}$. So we get that $d_{2^{e}}=y_{e+1}$ as desired. Part (iii) is proved. The proof of Lemma 3.9 is complete. 
Lemma 3.10. Let $p$ be an odd prime with $p \nmid a$, and let $D_{p}$ be defined as in (1.3).

(i). If $e \leq \nu_{p}(D)$, then $d_{p^{e}}=p^{\lceil e / 2\rceil}$. Further, $d_{p^{e}}$ is equal to the smallest positive root of the congruence $a^{2} x^{2}-D \equiv 0\left(\bmod p^{e}\right)$. Moreover, the distance between any two distinct solutions of the congruence $f(x) \equiv 0\left(\bmod p^{e}\right)$ is divisible by $p^{\lceil e / 2\rceil}$ if $e \geq 2$.

(ii). If e $>\nu_{p}(D)$ with either $\nu_{p}(D)$ being odd or $\left(\frac{D_{p}}{p}\right)=-1$, then $d_{p^{e}}=\infty$.

(iii). If $e>\nu_{p}(D)$ with $\nu_{p}(D)$ being even and $\left(\frac{D_{p}}{p}\right)=1$, then $d_{p^{e}}$ equals the smallest positive root of the congruence $a^{2} x^{2}-D \equiv 0\left(\bmod p^{e}\right)$.

Proof. (i). Obviously, part (i) is true if $e=1$. So we only need to show that part (i) holds for the case $e \geq 2$. Now let $e \geq 2$. For any given two integers $m_{1}$ and $m_{2}$ with $0 \leq m_{1} \neq m_{2}<p^{\lfloor e / 2\rfloor}$, we have $\left\langle-(2 a)^{-1} b\right\rangle_{p^{\lceil e / 2\rceil}}+m_{1} p^{\lceil e / 2\rceil}-\left(\left\langle-(2 a)^{-1} b\right\rangle_{p^{\lceil e / 2\rceil}}+\right.$ $\left.m_{2} p^{\lceil e / 2\rceil}\right) \equiv\left(m_{1}-m_{2}\right) p^{\lceil e / 2\rceil}\left(\bmod p^{e}\right)$. It is easy to derive from the above congruence and Lemma 3.3 (i) that the distance between any two distinct solutions of $f(x) \equiv 0$ $\left(\bmod p^{e}\right)$ is divisible by $p^{\lceil e / 2\rceil}$.

Pick two integers $m_{1}^{\prime}$ and $m_{2}^{\prime}$ in the interval $\left[0, p^{\lfloor e / 2\rfloor}\right)$ such that $m_{1}^{\prime}-m_{2}^{\prime}=1$. Thus $d_{p^{e}}=p^{\lceil e / 2\rceil}$. But $e \leq \nu_{p}(D)$. So $p^{\lceil e / 2\rceil}$ is the smallest solution of the congruence $a^{2} x^{2}-D \equiv 0\left(\bmod p^{e}\right)$. Part (i) is proved.

(ii). Since $e>\nu_{p}(D)$ with $\nu_{p}(D)$ being odd or $\left(\frac{D_{p}}{p}\right)=-1$, by Lemma 3.3 we know that $S\left(f, p^{e}\right)$ is empty. Thus $d_{p^{e}}=\infty$ as desired.

(iii). Since $e>\nu_{p}(D)$ with $\nu_{p}(D)$ being even and $\left(\frac{D_{p}}{p}\right)=1$, by Lemma 3.3 (iii) the congruence $a^{2} x^{2}-D \equiv 0\left(\bmod p^{e}\right)$ has exactly $2 p^{\frac{\nu_{p}(D)}{2}}$ roots in any complete residue system modulo $p^{e}$. Let $y_{e}$ be the smallest positive root of the congruence $a^{2} x^{2}-D \equiv 0$ $\left(\bmod p^{e}\right)$. Then Lemma $3.3\left(\right.$ iii) applied to the congruence $a^{2} x^{2}-D \equiv 0\left(\bmod p^{e}\right)$ gives that $y_{e} \in\left[1, p^{e-\frac{\nu_{p}(D)}{2}}\right]$ and $\nu_{p}\left(y_{e}\right)=\nu_{p}\left(X_{p^{e}}\right)=\frac{\nu_{p}(D)}{2}$. One can easily check that $\left\langle a y_{e}\right\rangle_{p^{e-\frac{\nu_{p}(D)}{2}}}$ and $\left\langle-a y_{e}\right\rangle_{p^{e-\frac{\nu_{p}(D)}{2}}}$ are the only two solutions of $x^{2}-D \equiv 0\left(\bmod p^{e}\right)$ in the interval $\left[1, p^{e-\frac{\nu_{p}(D)}{2}}\right]$. So by Lemma 3.3 (iii), the following set

$$
\left\{\left\langle \pm a y_{e}\right\rangle_{p^{e-\frac{\nu_{p}(D)}{2}}}+m p^{e-\frac{\nu_{p}(D)}{2}}: 0 \leq m<p^{\frac{\nu_{p}(D)}{2}}\right\}
$$

is exactly the set of all the solutions of the congruence $x^{2}-D \equiv 0\left(\bmod p^{e}\right)$ in the interval $\left[1, p^{e}\right]$. For any solution $x_{0}$ of $f\left(x_{0}\right) \equiv 0\left(\bmod p^{e}\right)$, one has $\left(2 a x_{0}+b\right)^{2} \equiv D$ $\left(\bmod p^{e}\right)$. By (3.6), we have $2 a x_{0}+b \equiv\left\langle \pm a y_{e}\right\rangle_{p^{e-\frac{\nu_{p}(D)}{2}}}+m p^{e-\frac{\nu_{p}(D)}{2}}\left(\bmod p^{e}\right)$ for some $0 \leq m<p^{e-\frac{\nu_{p}(D)}{2}}$, which implies that $2 a x_{0}+b \equiv a y_{e}$ or $-a y_{e}\left(\bmod p^{e-\frac{\nu_{p}(D)}{2}}\right)$.

Now we prove that $d_{p^{e}}=y_{e}$. We only need to give the the proof for the case $2 a x_{0}+b \equiv$ $a y_{e}\left(\bmod p^{e-\frac{\nu_{p}(D)}{2}}\right)$, since the proof for the case $2 a x_{0}+b \equiv-a y_{e}\left(\bmod 2^{e-\frac{\nu_{2}(D)}{2}}\right)$ is similar. Let now $2 a x_{0}+b \equiv a y_{e}\left(\bmod p^{e-\frac{\nu_{p}(D)}{2}}\right)$. Then $2 a x_{0}+b=a y_{e}+m p^{e-\frac{\nu_{2}(D)}{2}}$ for some integer $m$. Using the fact that $\nu_{p}\left(y_{e}\right)=\frac{\nu_{p}(D)}{2}$, we get $f\left(x_{0}-y_{e}\right) \equiv-\left(2 a x_{0}+b\right) y_{e}+$ $a y_{e}^{2} \equiv 0\left(\bmod p^{e}\right)$, i.e., $x_{0}-y_{e}$ is a solution of $f(x) \equiv 0\left(\bmod p^{e}\right)$. Note that $x_{0}$ is also a solution of $f(x) \equiv 0\left(\bmod p^{e}\right)$. Therefore, $d_{p^{e}} \leq d_{p^{e}}\left(x_{0}, x_{0}-y_{e}\right) \leq y_{e}$.

We can check that $\left\langle x_{0}\right\rangle_{p^{e-\frac{\nu_{p}(D)}{2}}},\left\langle x_{0}-y_{e}\right\rangle_{p^{e-\frac{\nu p(D)}{2}}}$ are the only two distinct solutions of $f(x) \equiv 0\left(\bmod p^{e}\right)$ in the interval $\left[1, p^{e-\frac{\nu_{p}(D)}{2}}\right]$. Thus by Lemma $3.3(\mathrm{iii}), S\left(f, p^{e}\right)$ is equal to the following union:

$$
\left\{\left\langle x_{0}\right\rangle_{p^{e-\frac{\nu_{p}(D)}{2}}}+m p^{e-\frac{\nu_{p}(D)}{2}}: 0 \leq m<p^{\frac{\nu_{p}(D)}{2}}\right\} \cup\left\{\left\langle x_{0}-y_{e}\right\rangle_{p^{e-\frac{\nu_{p}(D)}{2}}}+m p^{e-\frac{\nu_{p}(D)}{2}}: 0 \leq m<p^{\frac{\nu_{p}(D)}{2}}\right\} .
$$


Then $d_{p^{e}}$ must be of the form $\left\langle \pm\left\langle x_{0}\right\rangle_{p^{e-\frac{\nu_{p}(D)}{2}}} \mp\left\langle x_{0}-y_{e}\right\rangle_{p^{e-\frac{\nu_{p}(D)}{2}}}+m p^{e-\frac{\nu_{p}(D)}{2}}\right\rangle_{p^{e}}$ for some integer $m$. It follows from Lemma 3.3 (iii) that $\pm y_{e}+t p^{e-\frac{\nu_{p}(D)}{2}}$ is the root of $a^{2} x^{2}-D \equiv 0$

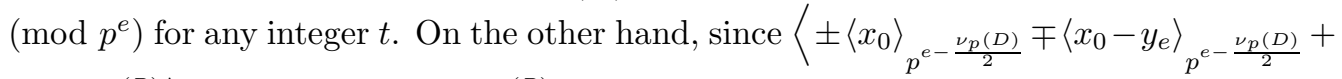
$\left.m p^{e-\frac{\nu_{p}(D)}{2}}\right\rangle_{p^{e}} \equiv \pm y_{e}\left(\bmod p^{e-\frac{\nu_{p}(D)}{2}}\right)$ for any integer $m, d_{p^{e}}$ is a solution of $a^{2} x^{2}-D \equiv 0$ $\left(\bmod p^{e}\right)$. Since $y_{e}$ is the smallest positive root of the congruence $a^{2} x^{2}-D \equiv 0\left(\bmod p^{e}\right)$, we have $d_{p^{e}} \geq y_{e}$. So $d_{p^{e}}=y_{e}$ as required. Part (iii) is proved. This completes the proof of Lemma 3.10.

Associated to $f$, we define a subset $\mathcal{K}_{f}$ of the set $\mathbb{N}^{*}$ of positive integers by $\mathcal{K}_{f}:=\{j \in$ $\mathbb{N}^{*}: D \neq a^{2} i^{2}$ for any integer $i$ such that $\left.1 \leq i \leq j\right\}$. Clearly $\mathcal{K}_{f}$ is empty if and only if $D=a^{2}$. Further, the condition that $D \neq a^{2} i^{2}$ for all integers $i$ with $1 \leq i \leq k$ presented in Theorem 1.2 is equivalent to that $\mathcal{K}_{f}$ is nonempty and $k \in \mathcal{K}_{f}$. The following result describes the form of $\mathcal{K}_{f}$.

Lemma 3.11. If $\mathcal{K}_{f}$ is nonempty, then we have that either $\mathcal{K}_{f}=\mathbb{N}^{*}$ or $\mathcal{K}_{f}=\{1, \ldots, l\}$, where $l$ is an integer such that $a^{2}(l+1)^{2}=D$.

Proof. If $\mathcal{K}_{f}=\mathbb{N}^{*}$, then Lemma 3.11 is true. If $\mathcal{K}_{f} \neq \mathbb{N}^{*}$, then the set $\mathbb{N}^{*} \backslash \mathcal{K}_{f}$ is nonempty. By the well-ordering principle (see, for example, page 13 of [1]), we know that $\mathbb{N}^{*} \backslash \mathcal{K}_{f}$ contains a smallest member, named $s_{0}=\min \left(\mathbb{N}^{*} \backslash \mathcal{K}_{f}\right)$. So $s_{0} \notin \mathcal{K}_{f}$. Suppose that $s_{0}=1$. Then $1 \notin \mathcal{K}_{f}$ and so $a^{2}=D$. It infers that $\mathcal{K}_{f}$ is empty, which is impossible since $\mathcal{K}_{f}$ is nonempty. Thus we have that $s_{0} \geq 2$ and all the integers $s$ with $s<s_{0}$ are belonging to $\mathcal{K}_{f}$, i.e., $\left\{1, \ldots, s_{0}-1\right\} \subseteq \mathcal{K}_{f}$.

On the other hand, since $s_{0} \notin \mathcal{K}_{f}$, there is an integer $s^{\prime}$ with $1 \leq s^{\prime} \leq s_{0}$ such that $a^{2} s^{\prime 2}-D=0$. Thus $s^{\prime} \in \mathbb{N}^{*} \backslash \mathcal{K}_{f}$. By the minimality of $s_{0}$, one has $s^{\prime}=s_{0}$. Hence $a^{2} s_{0}^{2}-D=0$, which implies that $s_{0}+j \notin \mathcal{K}_{f}$ for all nonnegative integers $j$. Therefore $\mathcal{K}_{f}=\left\{1, \ldots, s_{0}-1\right\}$ as desired. Hence Lemma 3.11 is proved.

Lemma 3.7 tells us that the sequence $\left\{d_{p^{e}}\right\}_{e=0}^{\infty}$ is nondecreasing. The following result describes a condition on $f$ which guarantees that $\left\{d_{p^{e}}\right\}_{e=0}^{\infty}$ is not a constant sequence.

Lemma 3.12. Let $\mathcal{K}_{f}$ be nonempty and $k \in \mathcal{K}_{f}$. Then for any prime $p$, there exists a unique nonnegative integer e such that $d_{p^{e}} \leq k<d_{p^{e+1}}$.

Proof. Let $\mathcal{K}_{f}$ be nonempty and $p$ be a prime. For any $k \in \mathcal{K}_{f}$, we define the subset $R_{p}(k)$ of $\mathbb{N}^{*}$ by $R_{p}(k):=\left\{i \in \mathbb{N}^{*}: k<d_{p^{i}}\right\}$.

If the set $R_{p}(k)$ is nonempty, then by the well-ordering principle [1], we know that $R_{p}(k)$ contains a smallest element, named $i_{0}=\min \left(R_{p}(k)\right)$. Letting $e=i_{0}-1$ gives the desired result $d_{p^{e}} \leq k<d_{p^{e+1}}$. The uniqueness of $e$ follows from Lemma 3.7. Thus we only need to show that $R_{p}(k)$ is nonempty for any prime $p$. In the following we show the equivalent statement that there is a positive integer $t$ such that $k<d_{p^{t}}$.

First let $p$ be a prime such that $p \mid a$. If $p \mid b$, then one can take $t=1$ since by Lemma 3.8 , we have $d_{p^{0}} \leq k<d_{p}=\infty$. If $p \nmid b$, then pick $t$ to be a positive integer such that $k<p^{t}$. However, Lemma 3.8 tells us that $d_{p^{t}}=p^{t}$. Hence $k<d_{p^{t}}$. The statement is true for the case $p \mid a$. Consequently, we let $p$ be a prime such that $p \nmid a$.

If $p=2$ and $D_{4} \not \equiv 1(\bmod 8)$, then we take $t=2\left\lfloor\frac{\nu_{2}(D)}{2}\right\rfloor+1$. Then by Lemma 3.9 (ii), we obtain that $d_{2^{t}}=\infty$ and thus $k<d_{2^{t}}$. The statement is true in this case. 
If $p$ is an odd prime with either $\nu_{p}(D)$ being odd or $\left(\frac{D_{p}}{p}\right)=-1$, then we take $t=$ $\nu_{p}(D)+1$. But $d_{p^{\nu_{p}(D)+1}}=\infty$ by Lemma 3.10 (ii). So we get the desired result $k<d_{p^{t}}$. The statement is proved in this case.

If either $p=2$ and $D_{4} \equiv 1(\bmod 8)$, or $p$ is an odd prime with $\nu_{p}(D)$ being even and $\left(\frac{D_{p}}{p}\right)=1$, then by the assumption that $\mathcal{K}_{f}$ is nonempty and Lemma 3.11 , we have either $\mathcal{K}_{f}=\mathbb{N}^{*}$, or $\mathcal{K}_{f}=\{1, \ldots, l\}$ for some positive integer $l$ satisfying $a^{2}(l+1)^{2}=D$. We take

$$
t=\max \left(\nu_{p}(D)+1, \log _{p}\left(a^{2} k^{2}+|D|\right)+1\right)
$$

if $\mathcal{K}_{f}=\mathbb{N}^{*}$, and

$$
t=\max \left(\nu_{p}(D)+1, \max _{1 \leq i \leq l}\left\{\nu_{p}\left(a^{2} i^{2}-D\right)\right\}\right)+1
$$

if $\mathcal{K}_{f}$ is a finite set. So $t>\nu_{p}(D)$. It then follows from Lemma 3.9 (iii) and Lemma 3.10 (iii) that $d_{p^{t}}$ is the smallest positive root of $a^{2} x^{2}-D \equiv 0\left(\bmod p^{t+1}\right)$ if $p=2$ and $d_{p^{t}}$ is the smallest positive root of $a^{2} x^{2}-D \equiv 0\left(\bmod p^{t}\right)$ if $p \neq 2$, respectively. Now we show that $d_{p^{t}}>k$.

For the former case $\mathcal{K}_{f}=\mathbb{N}^{*}$, we have that $a^{2} i^{2}-D \neq 0$ for any $i \in \mathbb{N}^{*}$. By (3.7), we have $t>\nu_{p}(D)$ and $p^{t}>a^{2} k^{2}+|D|$. Since $a^{2} d_{p^{t}}^{2}-D \equiv 0\left(\bmod p^{t}\right)$, we can write $a^{2} d_{p^{t}}^{2}-D=p^{t} u$ for some integer $u$. Then $u \neq 0$ because $a^{2} i^{2}-D \neq 0$ for any $i \in \mathbb{N}^{*}$. It then follows that $a^{2} d_{p^{t}}^{2} \geq p^{t}-|D|>a^{2} k^{2}$, which implies that $d_{p^{t}}>k$ as required.

For the latter case that $\mathcal{K}_{f}=\{1, \ldots, l\}$, we have $a^{2}(l+1)^{2}-D=0$. But by (3.8), we have $a^{2} i^{2}-D \neq 0\left(\bmod p^{t}\right)$ for all $1 \leq i \leq l$. Hence $l+1$ is the smallest positive root of the congruences $a^{2} x^{2}-D \equiv 0\left(\bmod p^{t}\right)$ and $a^{2} x^{2}-D \equiv 0\left(\bmod p^{t+1}\right)$. But $d_{p^{t}}$ is equal to the smallest positive root of the congruence $a^{2} x^{2}-D \equiv 0\left(\bmod p^{t+1}\right)$ if $p=2$, and $d_{p^{t}}$ is equal to the smallest positive root of the congruence $a^{2} x^{2}-D \equiv 0\left(\bmod p^{t}\right)$ if $p \neq 2$. Thus $d_{p^{t}}=l+1$. However, $k \leq l$ since $k \in \mathcal{K}_{f}$. So we obtain that $d_{p^{t}}>k$ as desired. The proof of Lemma 3.12 is complete.

Lemma 3.13. Let $\mathcal{K}_{f}$ be nonempty.

(i). Let $a$ be odd and $D_{4} \equiv 1(\bmod 8)$. If $k \in \mathcal{K}_{f}$ and there is an integer $e>\nu_{2}(D)$ such that $d_{2^{e}} \leq k<d_{2^{e+1}}$, then $\max _{1 \leq i \leq k}\left\{\nu_{2}\left(a^{2} i^{2}-D\right)\right\}=\nu_{2}\left(a^{2} d_{2^{e}}^{2}-D\right)=e+1$.

(ii). Let $p$ be an odd prime with $p \nmid a$. For any $k \in \mathcal{K}_{f}$ such that $d_{p^{e}} \leq k<d_{p^{e+1}}$ for some nonnegative integer $e$, we have $\max _{1 \leq i \leq k}\left\{\nu_{p}\left(a^{2} i^{2}-D\right)\right\}=\nu_{p}\left(a^{2} d_{p^{e}}^{2}-D\right)=e$.

Proof. (i). By Lemma 3.9 (iii), $d_{2 e}$ is the smallest solution of $a^{2} x^{2}-D \equiv 0\left(\bmod 2^{e+1}\right)$. Since $k \geq d_{2^{e}}$, we have $\max _{1 \leq i \leq k}\left\{\nu_{2}\left(a^{2} i^{2}-D\right)\right\} \geq \nu_{2}\left(a^{2} d_{2^{e}}^{2}-D\right) \geq e+1$. Part (iii) of Lemma 3.9 also tells us that $d_{2^{e+1}}$ is the smallest positive solution of $a^{2} x^{2}-D \equiv 0$ $\left(\bmod 2^{e+2}\right)$. Thus $\nu_{2}\left(a^{2} i^{2}-D\right)<e+2$ for all $1 \leq i<d_{2^{e+1}}$. It then follows immediately from $k<d_{2^{e+1}}$ that $\max _{1 \leq i \leq k}\left\{\nu_{2}\left(a^{2} i^{2}-D\right)\right\} \leq e+1$. Therefore we obtain the desired result $\max _{1 \leq i \leq k}\left\{\nu_{2}\left(a^{2} i^{2}-D\right)\right\}=\nu_{2}\left(a^{2} d_{2}^{2}-D\right)=e+1$. Part (i) is proved.

(ii). Since $k \geq d_{p^{e}}, d_{p^{e}}<\infty$. By parts (i) and (iii) of Lemma 3.10, we derive that $e \leq \nu_{p}\left(a^{2} d_{p^{e}}^{2}-D\right) \leq \max _{1 \leq i \leq k}\left\{\nu_{p}\left(a^{2} i^{2}-D\right)\right\}$. Noticing the facts that $k<d_{p^{e+1}}$ and $d_{p^{e+1}}$ is the smallest solution of $a^{2} x^{2}-D \equiv 0\left(\bmod p^{e+1}\right)$, we obtain $\max _{1 \leq i \leq k}\left\{\nu_{p}\left(a^{2} i^{2}-D\right)\right\} \leq$ $e$. It then follows that $\max _{1 \leq i \leq k}\left\{\nu_{p}\left(a^{2} i^{2}-D\right)\right\}=\nu_{p}\left(a^{2} d_{p^{e}}^{2}-D\right)=e$ as required. Part (ii) is true. So Lemma 3.13 is proved.

\section{4. $p$-Adic analysis of $g_{k, f}$ and determination of local periods}

In this section, we supply detailed local analysis to all eventually periodic arithmetic functions $g_{k, f}$ presented in Section 2. We always assume that $f$ is primitive throughout 
this section. Let $S_{k, f}(n):=\{f(n), f(n+1), \ldots, f(n+k)\}$ for any positive integer $n$. By the definition of $d_{p^{e+1}}$, we know that if $e$ is a nonnegative integer such that $d_{p^{e}} \leq k<d_{p^{e+1}}$, then there is at most one term divisible by $p^{e+1}$ in the set $S_{k, f}(n)$ for any positive integer $n$. We have

$$
\begin{aligned}
& g_{p, k, f}(n)=\sum_{m \in S_{k, f}(n)} v_{p}(m)-\max _{m \in S_{k, f}(n)}\left\{v_{p}(m)\right\} \\
& =\sum_{i=1}^{\infty} \#\left\{m \in S_{k, f}(n): \nu_{p}(m) \geq i\right\}-\sum_{i=1}^{\infty}\left(1 \text { if } \nu_{p}(m) \geq i \text { for some } m \in S_{k, f}(n)\right) \\
& =\sum_{i=1}^{\infty} \#\left\{m \in S_{k, f}(n): p^{i} \mid m\right\}-\sum_{i=1}^{\infty}\left(1 \text { if } p^{i} \text { divides some } m \in S_{k, f}(n)\right)=\sum_{i=1}^{\infty} h_{p, i}(n),
\end{aligned}
$$

where $h_{p, i}(n):=\max \left(0, \#\left\{m \in S_{k, f}(n): p^{i} \mid m\right\}-1\right)$. It follows that if the set $\{m \in$ $\left.S_{k, f}(n): p^{i} \mid m\right\}$ is nonempty, then $h_{p, i}(n):=\#\left\{m \in S_{k, f}(n): p^{i} \mid m\right\}-1$.

Lemma 4.1. Let $\mathcal{K}_{f}$ be nonempty and $p$ be a prime with $p \nmid a$. Let $k \in \mathcal{K}_{f}$ and e be the positive integer such that $d_{p^{e}} \leq k<d_{p^{e+1}}$. If $e \leq \nu_{p}(D)$, then there is a positive integer $n_{0}$ such that $g_{p, k, f}\left(n_{0}+p^{\lceil e / 2\rceil-1}\right) \neq g_{p, k, f}\left(n_{0}\right)$.

Proof. Let first $n$ be any positive integer and $e \leq \nu_{p}(D)$. Consider the difference $\Delta_{1}(n):=$ $g_{p, k, f}\left(n+p^{\lceil e / 2\rceil-1}\right)-g_{p, k, f}(n)$. Then to show Lemma 4.1, it suffices to find some suitable integer $n$ such that $\Delta_{1}(n) \neq 0$, which will be done in the following.

For any integer $i \geq e+1$, since $d_{p^{e}} \leq k<d_{p^{e+1}}$, there is at most one term divisible by $p^{i}$ in the set $S_{k, f}(n)$ for any positive integer $n$. Thus $\#\left\{m \in S_{k, f}(n): p^{i} \mid f(m)\right\} \leq 1$ and so $h_{p, i}(n)=0$ for any positive integer $n$. It follows from (4.1) that

$$
\Delta_{1}(n)=\sum_{i=1}^{e}\left(h_{p, i}\left(n+p^{\lceil e / 2\rceil-1}\right)-h_{p, i}(n)\right) .
$$

We claim that for any integers $m$ and $i$ with $1 \leq i \leq e$, we have

$$
\nu_{p}(f(n)) \geq i \Longleftrightarrow \nu_{p}\left(f\left(n+m p^{\lceil e / 2\rceil}\right) \geq i .\right.
$$

For the case $p=2$ and $2 \nmid a$, since $\nu_{2}(D) \geq e \geq 1$, we have that $\nu_{2}(D) \geq 2$ and $b$ is even. If $\nu_{2}(f(n)) \geq i$, then $\left(a n+\frac{b}{2}\right)^{2} \equiv \frac{D}{4}\left(\bmod 2^{i}\right)$. It follows that $\nu_{2}\left(a n+\frac{b}{2}\right) \geq\left\lceil\frac{i}{2}\right\rceil$ if $i \leq \nu_{2}(D)-2$, and $\nu_{2}\left(a n+\frac{b}{2}\right)=\left\lceil\frac{i}{2}\right\rceil-1$ if $i=\nu_{2}(D)-1$ or $\nu_{2}(D)$. Hence for any integer $m$, we obtain

$$
\begin{aligned}
\nu_{2}\left(f\left(n+m 2^{\lceil e / 2\rceil}\right)\right) & =\nu_{2}\left(f(n)+\left(a n+\frac{b}{2}\right) m 2^{\lceil e / 2\rceil+1}+a m^{2} \cdot 2^{2\lceil e / 2\rceil}\right) \\
& \geq \min \left\{i,\left\lceil\frac{i}{2}\right\rceil-1+\left\lceil\frac{e}{2}\right\rceil+1+\nu_{2}(m), 2\left\lceil\frac{e}{2}\right\rceil+2 \nu_{2}(m)\right\} \geq i .
\end{aligned}
$$

Similarly, one has

$$
\nu_{2}\left(f\left(n-m 2^{\lceil e / 2\rceil}\right)\right) \geq i .
$$

Conversely, if $\nu_{2}\left(f\left(n+m 2^{\left\lceil\frac{e}{2}\right\rceil}\right) \geq i\right.$, then we obtain by replacing $n$ with $n+m 2^{\lceil e / 2\rceil}$ in (4.4) that $\nu_{2}(f(n)) \geq i$. Therefore for any integers $m$ and $i$ with $1 \leq i \leq e$, we have

$$
\nu_{2}(f(n)) \geq i \Longleftrightarrow \nu_{2}\left(f\left(n+m 2^{\lceil e / 2\rceil}\right) \geq i .\right.
$$

That is, the claim is true for the case $p=2$ and $2 \nmid a$. 
For the case $p \neq 2$ and $p \nmid a$, if $\nu_{p}(f(n)) \geq i$, then it follows from $e \leq \nu_{p}(D)$ and $(2 a n+b)^{2} \equiv D\left(\bmod p^{i}\right)$ that $\nu_{p}(2 a n+b) \geq\lceil i / 2\rceil$, which implies that

$$
\nu_{p}\left(f\left(n \pm m p^{\lceil e / 2\rceil}\right)\right) \geq \min \left\{i,\lceil i / 2\rceil+\lceil e / 2\rceil+\nu_{p}(m), 2\lceil e / 2\rceil+2 \nu_{p}(m)\right\} \geq i .
$$

If $\nu_{p}\left(f\left(n+m p^{\lceil e / 2\rceil}\right)\right) \geq i$, replacing $n$ with $n+m p^{\lceil e / 2\rceil}$ in (4.6) , then $\nu_{p}(f(n))=\nu_{p}(f(n+$ $\left.\left.m p^{\lceil e / 2\rceil}-m p^{\lceil e / 2\rceil}\right)\right) \geq i$. Hence (4.3) holds in this case. The claim is proved.

Replacing $e$ by $2\lceil e / 2\rceil-2$, then (4.3) gives that for any given $1 \leq i \leq 2\lceil e / 2\rceil-2$ and any $0 \leq j \leq k, \nu_{p}(f(n+j)) \geq i \Longleftrightarrow \nu_{p}\left(f\left(n+j+p^{\lceil e / 2\rceil-1}\right) \geq i\right.$. Thus the number of terms divisible by $p^{i}$ in $S_{k, f}(n)$ is equal to that in $S_{k, f}\left(n+p^{\lceil e / 2\rceil-1}\right)$ for each $1 \leq i \leq 2\lceil e / 2\rceil-2$. It implies that $h_{p, i}\left(n+p^{\lceil e / 2\rceil-1}\right)=h_{p, i}(n)$ for each $1 \leq i \leq 2\lceil e / 2\rceil-2$. Therefore by (4.2), we derive that

$$
\Delta_{1}(n)=\sum_{i=2\lceil e / 2\rceil-1}^{e}\left(h_{p, i}\left(n+p^{\lceil e / 2\rceil-1}\right)-h_{p, i}(n)\right) .
$$

Since $k \geq d_{p^{e}}$ implying that $d_{p^{e}}<\infty$, by the definition of $d_{p^{e}}$ we know that $S\left(f, p^{e}\right)$ is nonempty. Define $x_{0}:=$ the smallest positive solution of the congruence $f(x) \equiv 0$ $\left(\bmod p^{e}\right)$. Then by Lemma $3.2(\mathrm{i})$-(ii) and Lemma $3.3(\mathrm{i})$, any term divisible by $p^{e}$ in the quadratic sequence $\{f(m)\}_{m=1}^{\infty}$ must be of the form $f\left(x_{0}+t p^{\lceil e / 2\rceil}\right)$ with $t \in \mathbb{N}$. But $\lceil e / 2\rceil=\lceil(e-1) / 2\rceil$ if $e$ is even. Thus by Lemma 3.2 (i)-(ii) and Lemma 3.3 (i), the terms divisible by $p^{e-1}$ are exactly the terms divisible by $p^{e}$ in the quadratic progression $\{f(m)\}_{m=1}^{\infty}$. Hence the terms divisible by $p^{e-1}$ are exactly the terms divisible by $p^{e}$ in the set $S_{k, f}(n)$ (resp. $S_{k, f}\left(n+p^{\lceil e / 2\rceil-1}\right)$ ) if $e$ is even. Namely, $\left\{\bar{m} \in S_{k, f}(m): p^{e-1}\right.$ | $\bar{m}\}=\left\{\bar{m} \in S_{k, f}(m): p^{e} \mid \bar{m}\right\}$ for $m=n, n+p^{\lceil e / 2\rceil-1}$. So $h_{p, e-1}(m)=h_{p, e}(m)$ for $m=n, n+p^{\lceil e / 2\rceil-1}$, which implies that $h_{p, e-1}\left(n+p^{\lceil e / 2\rceil-1}\right)-h_{p, e-1}(n)=h_{p, e}(n+$ $\left.p^{\lceil e / 2\rceil-1}\right)-h_{p, e}(n)$ if $e$ is even. It then follows from (4.7) that

$$
\Delta_{1}(n)=2^{\frac{1+(-1)^{e}}{2}}\left(h_{p, e}\left(n+p^{\lceil e / 2\rceil-1}\right)-h_{p, e}(n)\right)
$$

Since the terms divisible by $p^{e}$ in the sets $S_{k, f}(n)$ and $S_{k, f}\left(n+p^{\lceil e / 2\rceil-1}\right)$ are of the form $f\left(x_{0}+t p^{\lceil e / 2\rceil}\right)$ with $t \in \mathbb{N}$, in order to compute $\Delta_{1}(n)$, it is sufficient to compare the number of terms of the form $f\left(x_{0}+t p^{\lceil e / 2\rceil}\right)(t \in \mathbb{N})$ in the set $S_{k, f}(n)$ with that in the set $S_{k, f}\left(n+p^{\lceil e / 2\rceil-1}\right)$. By Lemma 3.9 (i) and Lemma $3.10(\mathrm{i}), d_{p^{e}}=p^{\lceil e / 2\rceil}$. But $k \geq d_{p^{e}}$. Thus $k \geq p^{\lceil e / 2\rceil}$. Since $\nu_{p}(k+1)<\lceil e / 2\rceil$, we can suppose that $k=k_{0} p^{\lceil e / 2\rceil}+r$ for unique two integers $k_{0}$ and $r$ with $k_{0} \geq 1$ and $0 \leq r \leq p^{\lceil e / 2\rceil}-2$.

If $0 \leq r<p^{\lceil e / 2\rceil}-p^{\lceil e / 2\rceil-1}$, then $p^{\lceil e / 2\rceil-1} \leq r+p^{\lceil e / 2\rceil-1}<p^{\lceil e / 2\rceil}$. Hence the number of integers $t$ such that $x_{0}+p^{\lceil e / 2\rceil-1} \leq x_{0}+t p^{\lceil e / 2\rceil} \leq x_{0}+k+p^{\lceil e / 2\rceil-1}$ is equal to $\left\lfloor\frac{k+p^{\lceil e / 2\rceil-1}}{p^{\mid e / 2\rceil}}\right\rfloor=k_{0}+\left\lfloor\frac{r+p^{\lceil e / 2\rceil-1}}{p^{\mid e / 2\rceil}}\right\rfloor=k_{0}$. So there are exactly $k_{0}$ terms divisible by $p^{e}$ in the set $S_{k, f}\left(x_{0}+p^{\lceil e / 2\rceil-1}\right)$. Thus $h_{p, e}\left(x_{0}+p^{\lceil e / 2\rceil-1}\right)=k_{0}-1$. Similarly, by counting the number of integers $t$ satisfying $x_{0} \leq x_{0}+t p^{\lceil e / 2\rceil} \leq x_{0}+k$, we get that the number of terms divisible by $p^{e}$ in $S_{k, f}\left(x_{0}\right)$ equals $\left\lfloor\frac{k}{p^{\mid e / 2 T}}\right\rfloor+1=k_{0}+1$ and so $h_{p, e}\left(x_{0}\right)=k_{0}$. Thus we derive from (4.8) that $\Delta_{1}\left(x_{0}\right)=-2^{\frac{1+(-1)^{e}}{2}}$.

If $p^{\lceil e / 2\rceil}-p^{\lceil e / 2\rceil-1} \leq r \leq p^{\lceil e / 2\rceil}-2$, then $p^{\lceil e / 2\rceil}-p^{\lceil e / 2\rceil-1}+1 \leq r+1 \leq p^{\lceil e / 2\rceil}-1$ and $p^{\lceil e / 2\rceil}+1 \leq r+p^{\lceil e / 2\rceil-1}+1 \leq p^{\lceil e / 2\rceil}+p^{\lceil e / 2\rceil-1}-1$. Therefore, by counting the number of integers $t$ such that $x_{0}+1 \leq x_{0}+t p^{\lceil e / 2\rceil} \leq x_{0}+k+1$ (resp. $x_{0}+p^{\lceil e / 2\rceil-1}+1 \leq x_{0}+t p^{\lceil e / 2\rceil} \leq$ $\left.x_{0}+p^{\lceil e / 2\rceil-1}+k+1\right)$, we deduce that $h_{p, e}\left(x_{0}+1\right)=\left\lfloor\frac{k+1}{p^{\mid e / 2\rceil}}\right\rfloor-1=k_{0}+\left\lfloor\frac{r+1}{p^{\lceil e / 2\rceil}}\right\rfloor-1=k_{0}-1$ and $h_{p, e}\left(x_{0}+p^{\lceil e / 2\rceil-1}+1\right)=\left\lfloor\frac{k+p^{\lceil e / 2\rceil-1}+1}{p^{p^{\lceil/ 2\rceil}}}\right\rfloor-1=k_{0}+\left\lfloor\frac{r+p^{\lceil e / 2\rceil-1}+1}{p^{\lceil e / 2\rceil}}\right\rfloor-1=k_{0}$. It then 
follows from (4.8) that $\Delta_{1}\left(x_{0}+1\right)=2^{\frac{1+(-1)^{e}}{2}}$. Thus the desired result follows immediately. This completes the proof of Lemma 4.1 .

With the help of (4.1), we can make a detailed local analysis to determine the local period $P_{p, k, f}$ for each prime factor $p$ of $B_{k}$. We have the following results.

Lemma 4.2. Let $p$ be a prime such that $p \mid a$. Then

$$
P_{p, k, f}=\left\{\begin{aligned}
p^{\nu_{p}\left(B_{k}\right)}, & \text { if } p \nmid b \text { and } \nu_{p}(k+1)<\nu_{p}\left(B_{k}\right), \\
1, & \text { otherwise. }
\end{aligned}\right.
$$

Proof. If $p \mid b$, then $p \nmid f(n)$ for any positive integer $n$ since $\operatorname{gcd}(a, b, c)=1$. In other words, $g_{p, k, f}(n)=0$ for any positive integer $n$. Thus $P_{p, k, f}=1$ as required. Lemma 4.2 is true if $p \mid b$.

Now we let $p \nmid b$. Then $p \nmid D=b^{2}-4 a c$ since $p \mid a$. It follows that $\nu_{p}\left(a^{2} n^{2}-D\right)=0$ for any positive integer $n$. Hence $\nu_{p}\left(B_{k}\right)=\nu_{p}\left(\operatorname{lcm}_{1 \leq i \leq k}\left\{i\left(a^{2} i^{2}-D\right)\right\}\right)=\max _{1 \leq i \leq k}\left\{\nu_{p}(i)\right\}=$ $\nu_{p}\left(L_{k}\right)$. By Lemma 3.1, there is exactly one term divisible by $p^{e}$ in any consecutive $p^{e}$ terms of the quadratic progression $\{f(n+m)\}_{m \in \mathbb{N}}$ for any given positive integers $e$ and $n$. Since $p^{\nu_{p}\left(L_{k}\right)} \leq k<p^{\nu_{p}\left(L_{k}\right)+1}$, it follows from Lemma 3.8 that $d_{p^{\nu_{p}\left(L_{k}\right)}} \leq k<d_{p^{\nu\left(L_{k}\right)+1}}$. Then there is at most one term divisible by $p^{\nu_{p}\left(L_{k}\right)+1}$ in $S_{k, f}(n)$ for any positive integer $n$. Consider the following two cases.

Case 1. $\nu_{p}(k+1) \geq \nu_{p}\left(B_{k}\right)=\nu_{p}\left(L_{k}\right)$. By Lemma 3.1, we deduce that there are exactly $\frac{k+1}{p^{e}}$ terms divisible by $p^{e}$ in $S_{k, f}(n)$ (resp. $S_{k, f}(n+1)$ ) for any positive integer $n$ and each $e \in\left\{1, \ldots, \nu_{p}\left(L_{k}\right)\right\}$. On the other hand, since there is at most one term divisible by $p^{\nu_{p}\left(L_{k}\right)+1}$ in $S_{k, f}(n)$ (resp. $S_{k, f}(n+1)$ ), we have by (4.1) that $g_{p, k, f}(n)=$ $\sum_{e=1}^{\nu_{p}\left(L_{k}\right)}\left(\frac{k+1}{p^{e}}-1\right)=g_{p, k, f}(n+1)$ for any positive integer $n$. Therefore $P_{p, k, f}=1$ as desired. Lemma 4.2 is proved in this case.

Case 2. $\nu_{p}(k+1)<\nu_{p}\left(B_{k}\right)=\nu_{p}\left(L_{k}\right)$. Evidently, $\nu_{p}\left(L_{k}\right) \geq 1$. Since there is at most one term divisible by $p^{\nu_{p}\left(L_{k}\right)+1}$ in $S_{k, f}(n)$ for any positive integer $n$, we have $h_{p, e}(n)=0$ if $e \geq \nu_{p}\left(L_{k}\right)+1$. Thus we can deduce from (4.1) that $g_{p, k, f}(n)=\sum_{e=1}^{\nu_{p}\left(L_{k}\right)} h_{p, e}(n)$.

By Lemma 2.2, $p^{\nu_{p}\left(L_{k}\right)}$ is a period of $g_{p, k, f}$. So it remains to prove that $p^{\nu_{p}\left(L_{k}\right)-1}$ is not a period of $g_{p, k, f}$. For any integer $e$ such that $1 \leq e \leq \nu_{p}\left(L_{k}\right)-1$, since $f\left(n+p^{\nu_{p}\left(L_{k}\right)-1}\right) \equiv$ $f(n)\left(\bmod p^{e}\right)$ for any positive integer $n, p^{\nu_{p}\left(L_{k}\right)-1}$ is a period of $h_{p, e}$. Hence we only need to prove that $p^{\nu_{p}\left(L_{k}\right)-1}$ is not a period of $h_{p, \nu_{p}\left(L_{k}\right)}$. Since $\nu_{p}(k+1)<\nu_{p}\left(L_{k}\right)$, we can pick an $r \in\left\{0,1, \ldots, p^{\nu_{p}\left(L_{k}\right)}-2\right\}$ such that $k \equiv r\left(\bmod p^{\nu_{p}\left(L_{k}\right)}\right)$.

Subcase 2.1. $0 \leq r<p^{\nu_{p}\left(L_{k}\right)}-p^{\nu_{p}\left(L_{k}\right)-1}$. Then by Lemma 3.1, we can choose a positive integer $n_{0}$ such that $f\left(n_{0}\right) \equiv 0\left(\bmod p^{\nu_{p}\left(L_{k}\right)}\right)$. And so the terms divisible by $p^{\nu_{p}\left(L_{k}\right)}$ in the quadratic sequence $\left\{f\left(n_{0}+i\right)\right\}_{i \in \mathbb{N}}$ must be of the form $f\left(n_{0}+t p^{\nu_{p}\left(L_{k}\right)}\right)$ for

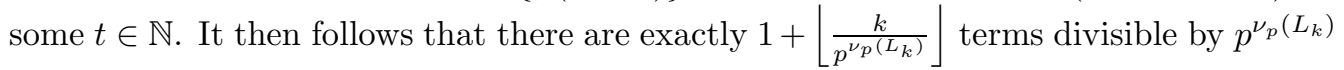
in $S_{k, f}\left(n_{0}\right)$ and there are exactly $\left\lfloor\frac{k+p^{\nu p\left(L_{k}\right)-1}}{p^{\nu p\left(L_{k}\right)}}\right\rfloor=\left\lfloor\frac{k-r}{p^{\nu p\left(L_{k}\right)}}\right\rfloor+\left\lfloor\frac{p^{\nu p\left(L_{k}\right)-1}+r}{p^{\nu p\left(L_{k}\right)}}\right\rfloor=\left\lfloor\frac{k}{\left.p^{\nu p\left(L_{k}\right)}\right\rfloor}\right.$ terms divisible by $p^{\nu_{p}\left(L_{k}\right)}$ in $S_{k, f}\left(n_{0}+p^{\nu_{p}\left(L_{k}\right)-1}\right)$, where the last equality is derived from $k \equiv r\left(\bmod p^{\nu_{p}\left(L_{k}\right)}\right)$ and $0 \leq r<p^{\nu_{p}\left(L_{k}\right)}-p^{\nu_{p}\left(L_{k}\right)-1}$. Thus $h_{p, \nu_{p}\left(L_{k}\right)}\left(n_{0}\right)=\left\lfloor\frac{k}{\left.p^{\nu p\left(L_{k}\right)}\right\rfloor=}\right.$ $h_{p, \nu_{p}\left(L_{k}\right)}\left(n_{0}+p^{\nu_{p}\left(L_{k}\right)-1}\right)+1$. That is, $p^{\nu_{p}\left(L_{k}\right)-1}$ is not a period of $h_{p, \nu_{p}\left(L_{k}\right)}$.

Subcase 2.2. $p^{\nu_{p}\left(L_{k}\right)}-p^{\nu_{p}\left(L_{k}\right)-1} \leq r \leq p^{\nu_{p}\left(L_{k}\right)}-2$. Again by Lemma 3.1, we can pick a suitable positive integer $m_{0}$ such that $f\left(m_{0}+p^{\nu_{p}\left(L_{k}\right)-1}-1\right) \equiv 0\left(\bmod p^{\nu_{p}\left(L_{k}\right)}\right)$. It follows that the terms divisible by $p^{\nu_{p}\left(L_{k}\right)}$ in the quadratic sequence $\left\{f\left(m_{0}+i\right)\right\}_{i \in \mathbb{N}}$ must be of the form $f\left(m_{0}+p^{\nu_{p}\left(L_{k}\right)-1}-1+s p^{\nu_{p}\left(L_{k}\right)}\right)$ for some $s \in \mathbb{N}$. Since $k \equiv r\left(\bmod p^{\nu_{p}\left(L_{k}\right)}\right)$ 
and $p^{\nu_{p}\left(L_{k}\right)}-p^{\nu_{p}\left(L_{k}\right)-1} \leq r \leq p^{\nu_{p}\left(L_{k}\right)}-2$, we can derive that the number of terms

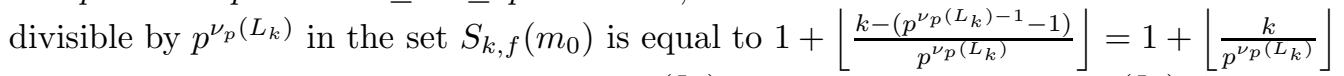
and the number of terms divisible by $p^{\nu_{p}\left(L_{k}\right)}$ in the set $S_{k, f}\left(m_{0}+p^{\nu_{p}\left(L_{k}\right)-1}\right)$ equals $\left\lfloor\frac{k+1}{p^{\nu p\left(L_{k}\right)}}\right\rfloor=\left\lfloor\frac{k}{p^{\nu p\left(L_{k}\right)}}\right\rfloor$. Thus $h_{p, \nu_{p}\left(L_{k}\right)}\left(m_{0}\right)=h_{p, \nu_{p}\left(L_{k}\right)}\left(m_{0}+p^{\nu_{p}\left(L_{k}\right)-1}\right)+1$. Namely, $p^{\nu_{p}\left(L_{k}\right)-1}$ is not a period of $h_{p, \nu_{p}\left(L_{k}\right)}$ as required. The proof of Lemma 4.2 is complete.

Now we need only to handle the even prime 2 and the odd prime $p$ with $p \nmid a$, respectively. We first consider the case $2 \nmid a$. Since $D_{4} \equiv 1(\bmod 4)$ if $\nu_{2}(D)=0$, we have that $\nu_{2}(D) \geq 1$ if $e=\nu_{2}(D)$ with $D_{4} \not \equiv 1(\bmod 4)$. Therefore, if either $e=\nu_{2}(D)$ with $D_{4} \not \equiv 1(\bmod 4)$ or $e>\nu_{2}(D)$ with $D_{4} \not \equiv 1(\bmod 8)$, then by Lemma 3.9 (ii), $d_{2^{e}}=\infty$. But there is no integer $k$ such that $k \geq d_{2^{e}}$ for such integers $e$. So one only needs to consider the cases occurred exactly in Lemma 4.3.

Lemma 4.3. Let $a$ be odd and $\mathcal{K}_{f}$ be nonempty. Let $k \in \mathcal{K}_{f}$ and $e$ be the nonnegative integer such that $d_{2^{e}} \leq k<d_{2^{e+1}}$. Each of the following is true.

(i). If $e=\nu_{2}(D)$ with $D_{4} \equiv 1(\bmod 4)$ or $e \leq 2\left\lfloor\frac{\nu_{2}(D)}{2}\right\rfloor-1$, then

$$
P_{2, k, f}=\left\{\begin{aligned}
2^{\lceil e / 2\rceil}, & \text { if } \nu_{2}(k+1)<\lceil e / 2\rceil, \\
1, & \text { if } \nu_{2}(k+1) \geq\lceil e / 2\rceil .
\end{aligned}\right.
$$

(ii). If $e>\nu_{2}(D)$ with $D_{4} \equiv 1(\bmod 8)$, then $P_{2, k, f}=2^{e-\frac{\nu_{2}(D)}{2}}$.

Proof. Since $d_{2^{e}} \leq k<d_{2^{e+1}}$, there is at most one term divisible by $2^{e+1}$ in $S_{k, f}(n)$ for any positive integer $n$. It follows from (4.1) that $2^{0}=1$ is the smallest period of $g_{2, k, f}$ if $e=0$. So it remains to treat with the case $e \geq 1$. Let now $e \geq 1$ and $n \geq 1$ be an

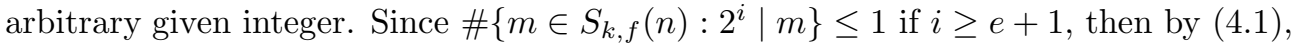

$$
g_{2, k, f}(n)=\sum_{i=1}^{e} h_{2, i}(n) \text {, }
$$

where

$h_{2, i}(n)=\max \left(0, \#\left\{m \in S_{k, f}(n): 2^{i} \mid m\right\}-1\right)=\max \left(0, \#\left\{0 \leq j \leq k: 2^{i} \mid f(n+j)\right\}-1\right)$.

Clearly, $h_{2, i}(n)=\#\left\{0 \leq j \leq k: 2^{i} \mid f(n+j)\right\}-1$ if there is at least one term divisible by $2^{i}$ in $S_{k, f}(n)$.

(i). Since $e=\nu_{2}(D) \geq 1$ with $D_{4} \equiv 5(\bmod 8)$ or $e \leq 2\left\lfloor\frac{\nu_{2}(D)}{2}\right\rfloor-1$, we have $\nu_{2}(D) \geq 2$ and $b$ is even. If $\nu_{2}(k+1) \geq\lceil e / 2\rceil$, comparing $S_{k, f}(n)$ with $S_{k, f}(n+1)$, we find that their distinct terms are $f(n)$ and $f(n+k+1)$. Since $\nu_{2}(k+1) \geq\lceil e / 2\rceil$, we have $k+1=m_{0} 2^{\lceil e / 2\rceil}$ for some positive integer $m_{0}$. From (4.5), we deduce that for any given integer $i$ with $1 \leq i \leq e, \nu_{2}(f(n)) \geq i$ if and only if $\nu_{2}(f(n+k+1)) \geq i$. Thus the number of terms divisible by $2^{i}$ in $S_{k, f}(n)$ is equal to the number of terms divisible by $2^{i}$ in $S_{k, f}(n+1)$ for each $i \in\{1, \ldots, e\}$. Hence by (4.9), we obtain that $g_{2, k, f}(n)=g_{2, k, f}(n+1)$ for any positive integer $n$, which implies that $P_{2, k, f}=1$. Part (i) is true in this case.

In what follows we let $\nu_{2}(k+1)<\lceil e / 2\rceil$. It follows from (4.5) that for any given $1 \leq i \leq e$ and for any $0 \leq j \leq k, \nu_{2}(f(n+j)) \geq i \Longleftrightarrow \nu_{2}\left(f\left(n+j+2^{\lceil e / 2\rceil}\right) \geq i\right.$. In other words, the number of terms divisible by $2^{i}$ in $S_{k, f}\left(n+2^{\lceil e / 2\rceil}\right)$ is equal to the number of terms divisible by $2^{i}$ in $S_{k, f}(n)$ for each $1 \leq i \leq e$. So $g_{2, k, f}\left(n+2^{\lceil e / 2\rceil}\right)=g_{2, k, f}(n)$ for any positive integer $n$. This infers that $2^{\lceil e / 2\rceil}$ is a period of $g_{2, k, f}$. On the other hand, by Lemma 4.1 one knows that there is a positive integer $n_{0}$ such that $g_{2, k, f}\left(n_{0}+2^{\lceil e / 2\rceil-1}\right)=$ $g_{2, k, f}\left(n_{0}\right)$. Thus $2^{\lceil e / 2\rceil-1}$ is not a period of $g_{2, k, f}$. Therefore $2^{\lceil e / 2\rceil}$ is the smallest period of $g_{2, k, f}$. Part (i) is proved. 
(ii). Since $D_{4}$ is odd, $\nu_{2}(D)$ is even. First, we prove that $2^{e-\frac{\nu_{2}(D)}{2}}$ is a period of $g_{2, k, f}$. Since $f\left(m+2^{e}\right) \equiv f(m)\left(\bmod 2^{i}\right)$, we get that $h_{2, i}\left(m+2^{e}\right)=h_{2, i}(m)$ for any integers $m$ and $i$ with $0 \leq i \leq e$. So by (4.2), $g_{2, k, f}\left(m+2^{e}\right)=g_{2, k, f}(m)$ for any integer $m$, i.e., $2^{e}=2^{e-\frac{\nu_{2}(D)}{2}}$ is a period of $g_{2, k, f}$ if $\nu_{2}(D)=0$.

Now let $\nu_{2}(D) \geq 2$. Then $b$ is even. Let $l$ be any given positive integer with $l \geq \nu_{2}(D)$, and let $i \in\{1, \ldots, l\}$ and $j \in\{0, \ldots, k\}$. If

$$
\nu_{2}(f(n+j)) \geq i,
$$

then $\left(a(n+j)+\frac{b}{2}\right)^{2} \equiv \frac{D}{4}\left(\bmod 2^{i}\right)$, which implies that $\nu_{2}\left(a(n+j)+\frac{b}{2}\right) \geq \min \left\{\frac{\nu_{2}(D)}{2}-\right.$ $\left.1,\left\lceil\frac{i}{2}\right\rceil\right\}$. But $\min \left\{\frac{\nu_{2}(D)}{2}-1,\left\lceil\frac{i}{2}\right\rceil\right\} \geq\left\lceil\frac{i}{2}\right\rceil$ if $i \leq \nu_{2}(D)-2$, and $\min \left\{\frac{\nu_{2}(D)}{2}-1,\left\lceil\frac{i}{2}\right\rceil\right\}=$ $\frac{\nu_{2}(D)}{2}-1$ if $i \geq \nu_{2}(D)-1$. It then follows that

$$
\begin{aligned}
& \nu_{2}\left(f\left(n+j+2^{l-\frac{\nu_{2}(D)}{2}}\right)\right)=\nu_{2}\left(f(n+j)+\left(a(n+j)+\frac{b}{2}\right) 2^{l-\frac{\nu_{2}(D)}{2}+1}+a 2^{2 l-\nu_{2}(D)}\right) \\
& \geq \min \left\{i, l-\frac{\nu_{2}(D)}{2}+1+\min \left\{\frac{\nu_{2}(D)}{2}-1,\left\lceil\frac{i}{2}\right\rceil\right\}, 2 l-\nu_{2}(D)\right\} \geq i .
\end{aligned}
$$

Similarly, we have

$$
\nu_{2}\left(f\left(n+j-2^{l-\frac{\nu_{2}(D)}{2}}\right)\right) \geq i
$$

If $\nu_{2}\left(f\left(n+j+2^{l-\frac{\nu_{2}(D)}{2}}\right)\right) \geq i$, then the process of (4.11) derived from (4.10) with $n$ replaced by $n+2^{l-\frac{\nu_{2}(D)}{2}}$ gives us that $\nu_{2}(f(n+j)) \geq i$. Therefore, if $l$ is an integer with $l \geq \nu_{2}(D)$, then for any integers $i$ and $j$ with $1 \leq i \leq l$ and $0 \leq j \leq k$, we have

$$
\nu_{2}(f(n+j)) \geq i \Longleftrightarrow \nu_{2}\left(f\left(n+j+2^{l-\frac{\nu_{2}(D)}{2}}\right)\right) \geq i \text {. }
$$

Since $e>\nu_{2}(D)$, the number of terms divisible by $2^{i}$ in $S_{k, f}\left(n+2^{e-\frac{\nu_{2}(D)}{2}}\right)$ is equal to the number of terms divisible by $2^{i}$ in $S_{k, f}(n)$ and so $h_{2, i}(n)=h_{2, i}\left(n+2^{e-\frac{\nu_{2}(D)}{2}}\right)$ for each $1 \leq i \leq e$. Thus by (4.9), we have $g_{2, k, f}\left(n+2^{e-\frac{\nu_{2}(D)}{2}}\right)=g_{2, k, f}(n)$ for any positive integer $n$. So $2^{e-\frac{\nu_{2}(D)}{2}}$ is a period of $g_{2, k, f}$.

In the following, we prove that $2^{e-\frac{\nu_{2}(D)}{2}}-1$ is not the period of $g_{2, k, f}$. It suffices to show that $\Delta_{2}(n) \neq 0$ for some integer $n$, where

$$
\Delta_{2}(n):=g_{2, k, f}\left(n+2^{e-\frac{\nu_{2}(D)}{2}-1}\right)-g_{2, k, f}(n)=\sum_{i=1}^{e}\left(h_{2, i}\left(n+2^{e-\frac{\nu_{2}(D)}{2}-1}\right)-h_{2, i}(n)\right) .
$$

Since $e-1 \geq \nu_{2}(D)$, replacing $e$ by $e-1$ in (4.12), one gets that for any integers $i$ and $j$ with $1 \leq i \leq e-1$ and $0 \leq j \leq k, \nu_{2}(f(n+j)) \geq i$ if and only if $\nu_{2}\left(f\left(n+j+2^{e-\frac{\nu_{2}(D)}{2}-1}\right)\right) \geq$ $i$. In other words, the number of terms divisible by $2^{i}$ in $S_{k, f}(n)$ is equal to that in $S_{k, f}(n+$ $2^{e-\frac{\nu_{2}(D)}{2}-1}$ ) for each $1 \leq i \leq e-1$. Thus $\sum_{i=1}^{e-1} h_{2, i}(n)=\sum_{i=1}^{e-1} h_{2, i}\left(n+2^{e-\frac{\nu_{2}(D)}{2}-1}\right)$. It then follows from (4.13) that $\Delta_{2}(n)=h_{2, e}\left(n+2^{e-\frac{\nu_{2}(D)}{2}}-1\right)-h_{2, e}(n)$. Therefore, our final task is to find some suitable integer $n$ such that

$$
h_{2, e}\left(n+2^{e-\frac{\nu_{2}(D)}{2}-1}\right) \neq h_{2, e}(n) .
$$

Since $a$ is odd and $D_{4} \equiv 1(\bmod 8)$, we have $\nu_{2}\left(a^{2}\left(2^{\frac{\nu_{2}(D)}{2}}\right)^{2}-D\right)=\nu_{2}(D)+\nu_{2}\left(a^{2}-\right.$ $\left.D_{4}\right) \geq \nu_{2}(D)+3$, which means that $a^{2}\left(2^{\frac{\nu_{2}(D)}{2}}\right)^{2}-D \equiv 0\left(\bmod 2^{\nu_{2}(D)+3}\right)$. We can easily check that $2^{\frac{\nu_{2}(D)}{2}}$ is the smallest solution of the congruence $a^{2} x^{2}-D \equiv 0\left(\bmod 2^{i}\right)$ for 
any $i$ with $\nu_{2}(D) \leq i \leq \nu_{2}(D)+3$. Then by parts (i) and (iii) of Lemma 3.8, we derive that $d_{2^{\nu_{2}(D)-1}}=d_{2^{\nu_{2}(D)}}=d_{2^{\nu_{2}(D)+1}}=d_{2^{\nu_{2}(D)+2}}=2^{\frac{\nu_{2}(D)}{2}}$. Since $d_{2^{e}} \leq k<d_{2^{e+1}}$ and $e>\nu_{2}(D)$, we have $e \geq \nu_{2}(D)+2$. But $D_{4} \equiv 1(\bmod 8)$, then by part (iv) of Lemma 3.2, $S\left(f, 2^{e}\right)$ is nonempty.

We claim that for any $i \geq e, d_{2^{i}}<2^{i-\frac{\nu_{2}(D)}{2}-1}$. In fact, by Lemma 3.9 (iii), $d_{2^{i}}$ equals the smallest positive solution of $a^{2} x^{2}-D \equiv 0\left(\bmod 2^{i+1}\right)$. Then $\nu_{2}\left(d_{2^{i}}\right)=\frac{\nu_{2}(D)}{2}$. So $\nu_{2}\left(a^{2}\left(2^{i-\frac{\nu_{2}(D)}{2}}-d_{2^{i}}\right)^{2}-D\right) \geq i+1$, and hence $2^{i-\frac{\nu_{2}(D)}{2}}-d_{2^{i}}$ is also a solution of $a^{2} x^{2}-D \equiv 0\left(\bmod 2^{i+1}\right)$. From the minimality of $d_{2^{i}}$, we get that $2^{i-\frac{\nu_{2}(D)}{2}}-d_{2^{i}} \geq d_{2^{i}}$. But $2^{i-\frac{\nu_{2}(D)}{2}}-d_{2^{i}} \neq d_{2^{i}}$. Otherwise, $\nu_{2}\left(d_{2^{i}}\right)=i-\frac{\nu_{2}(D)}{2}-1 \geq \nu_{2}(D)+2-\frac{\nu_{2}(D)}{2}-1=$ $\frac{\nu_{2}(D)}{2}+1$, which is a contradiction since $\nu_{2}\left(d_{2^{i}}\right)=\frac{\nu_{2}(D)}{2}$. So $d_{2^{i}}<2^{i-\frac{\nu_{2}(D)}{2}-1}$. The claim is proved. From the claim, we know that $d_{2^{e}}<2^{e-\frac{\nu_{2}(D)}{2}-1}$ and $d_{2^{e+1}}<2^{e-\frac{\nu_{2}(D)}{2}}$. Thus $k<d_{2^{e+1}}<2^{e-\frac{\nu_{2}(D)}{2}}$.

If either $d_{2^{e+1}}>2^{e-\frac{\nu_{2}(D)}{2}-1}$ and $k<2^{e-\frac{\nu_{2}(D)}{2}-1}$ or $d_{2^{e+1}} \leq 2^{e-\frac{\nu_{2}(D)}{2}-1}$, then $d_{2^{e}} \leq k<$ $2^{e-\frac{\nu_{2}(D)}{2}-1}$. Since $S\left(f, 2^{e}\right)$ is nonempty, by Lemma 3.6 we can choose a positive integer $n_{0}$ such that $\nu_{2}\left(f\left(n_{0}\right)\right) \geq e$ and $\nu_{2}\left(f\left(n_{0}+d_{2^{e}}\right)\right) \geq e$. By Lemma 3.2 (iii), the terms divisible by $2^{e}$ in the quadratic progression $\left\{f\left(n_{0}+i\right)\right\}_{i \in \mathbb{N}}$ must be of the form $f\left(n_{0}+t_{1} 2^{e-\frac{\nu_{2}(D)}{2}}\right)$ or $f\left(n_{0}+d_{2^{e}}+t_{2} 2^{e-\frac{\nu_{2}(D)}{2}}\right)$, where $t_{1}, t_{2} \in \mathbb{N}$. On the one hand, since $d_{2^{e}} \leq k<2^{e-\frac{\nu_{2}(D)}{2}-1}$, $f\left(n_{0}\right)$ and $f\left(n_{0}+d_{2^{e}}\right)$ are the only two terms divisible by $2^{e}$ in $S_{k, f}\left(n_{0}\right)$. On the other hand, since $n_{0}+d_{2^{e}}<n_{0}+2^{e-\frac{\nu_{2}(D)}{2}-1}+j \leq n_{0}+k+2^{e-\frac{\nu_{2}(D)}{2}-1}<n_{0}+2^{e-\frac{\nu_{2}(D)}{2}}$ for all $0 \leq j \leq k$, there is no term divisible by $2^{e}$ in the set $S_{k, f}\left(n_{0}+2^{e-\frac{\nu_{2}(D)}{2}-1}\right)$. It follows that $h_{2, e}\left(n_{0}+2^{e-\frac{\nu_{2}(D)}{2}-1}\right)=0$ and $h_{2, e}\left(n_{0}\right)=1$. So (4.14) is true in this case.

If $k \geq 2^{e-\frac{\nu_{2}(D)}{2}-1}$, then it follows from Lemma 3.6 and the fact that $S\left(f, 2^{e}\right)$ is nonempty that there is a positive integer $n_{1}$ so that $\nu_{2}\left(f\left(n_{1}+2^{e-\frac{\nu_{2}(D)}{2}-1}-1-d_{2^{e}}\right)\right) \geq e$ and $\nu_{2}\left(f\left(n_{1}+2^{e-\frac{\nu_{2}(D)}{2}-1}-1\right)\right) \geq e$. Hence Lemma 3.2 (iii) tells us that the terms divisible by $2^{e}$ in the quadratic progression $\left\{f\left(n_{1}+i\right)\right\}_{i \in \mathbb{N}}$ should be of the form

$$
f\left(n_{1}+2^{e-\frac{\nu_{2}(D)}{2}-1}-1-d_{2^{e}}+t_{1} 2^{e-\frac{\nu_{2}(D)}{2}}\right)
$$

or

$$
f\left(n_{1}+2^{e-\frac{\nu_{2}(D)}{2}-1}-1+t_{2} 2^{e-\frac{\nu_{2}(D)}{2}}\right),
$$

where $t_{1}, t_{2} \in \mathbb{N}$. Since $k<d_{2^{e+1}}$ and $d_{2^{e+1}}<2^{e-\frac{\nu_{2}(D)}{2}}$, we have $2^{e-\frac{\nu_{2}(D)}{2}-1} \leq j+$ $2^{e-\frac{\nu_{2}(D)}{2}-1}<2^{e-\frac{\nu_{2}(D)}{2}}+2^{e-\frac{\nu_{2}(D)}{2}-1}-1$ for all $j \in\{0,1, \ldots, k\}$. Therefore, there is at most one term of the form (4.15) with $t_{1} \in \mathbb{N}$ and no term of the form (4.16) with $t_{2} \in \mathbb{N}$ in the set $S_{k, f}\left(n_{1}+2^{e-\frac{\nu_{2}(D)}{2}-1}\right)$. Since $k \geq 2^{e-\frac{\nu_{2}(D)}{2}-1}$ and $d_{2^{e}}<2^{e-\frac{\nu_{2}(D)}{2}}-1$, $f\left(n_{1}+2^{e-\frac{\nu_{2}(D)}{2}-1}-1\right)$ and $f\left(n_{1}+2^{e-\frac{\nu_{2}(D)}{2}-1}-1-d_{2^{e}}\right)$ are the only two terms divisible by $2^{e}$ in the set $S_{k, f}\left(n_{1}\right)$. So $h_{2, e}\left(n_{1}\right)=1$ and $h_{2, e}\left(n_{1}+2^{e-\frac{\nu_{2}(D)}{2}}\right)=0$, which implies that (4.14) holds in the case. This concludes that $2^{e-\frac{\nu_{2}(D)}{2}}$ is the smallest period of $g_{2, k, f}$.

This completes the proof of Lemma 4.3.

In what follows, we treat with all the odd primes $p$ with $p \nmid 2 a$ and $p \mid B_{k}$.

Lemma 4.4. Let $\mathcal{K}_{f}$ be nonempty and $p$ be an odd prime with $p \nmid a$. Let $k \in \mathcal{K}_{f}$ ande be the nonnegative integer such that $d_{p^{e}} \leq k<d_{p^{e+1}}$. Then 


$$
P_{p, k, f}=\left\{\begin{aligned}
1, & \text { if either } e \leq \nu_{p}(D) \text { and } \nu_{p}(k+1) \geq\lceil e / 2\rceil, \\
& \text { or } e>\nu_{p}(D) \text { and } \nu_{p}(k+1) \geq e-\nu_{p}(D) / 2, \\
p^{\lceil e / 2\rceil}, & \text { if } e \leq \nu_{p}(D) \text { and } \nu_{p}(k+1)<\lceil e / 2\rceil, \\
p^{e-\nu_{p}(D) / 2}, & \text { if } e>\nu_{p}(D) \text { and } \nu_{p}(k+1)<e-\nu_{p}(D) / 2 .
\end{aligned}\right.
$$

Proof. Let $n \geq 1$ be any positive integer. Since $d_{p^{e}} \leq k<d_{p^{e+1}}$, there is at most one term divisible by $p^{e+1}$ in the set $S_{k, f}(n)$ for any positive integer $n$. It follows from (4.1) that

$$
g_{p, k, f}(n)=\sum_{i=1}^{e} h_{p, i}(n),
$$

where $h_{p, i}(n)=\#\left\{0 \leq j \leq k: p^{i} \mid f(n+j)\right\}-1$ if there is at least one term divisible by $p^{i}$ in $S_{k, f}(n)$. Otherwise, $h_{p, i}(n)=0$. Thus $g_{p, k, f}(n)=0$ for any positive integer $n$, and so $P_{p, k, f}=1$ if $e=0$.

In what follows we let $e \geq 1$. Note that if $e>\nu_{p}(D)$ and $d_{p^{e}}<\infty$, then by parts (ii) and (iii) of Lemma 3.10, we know that $\nu_{p}(D)$ is even and $\left(D_{p} / p\right)=1$ for such primes $p$.

First we show that if $l \geq \nu_{p}(D)$ is an integer, then for any integers $m$ and $i$ with $1 \leq i \leq l$, we have

$$
\nu_{p}(f(n)) \geq i \Longleftrightarrow \nu_{p}\left(f\left(n+m p^{l-\frac{\nu_{p}(D)}{2}}\right) \geq i .\right.
$$

In fact, by (4.3), we know that for any integers $m$ and $i$ with $1 \leq i \leq \nu_{p}(D)$,

$$
\nu_{p}(f(n)) \geq i \Longleftrightarrow \nu_{p}\left(f\left(n+m p^{\nu_{p}(D)}\right) \geq i\right. \text {. }
$$

Since $l \geq \nu_{p}(D), p^{l-\frac{\nu_{p}(D)}{2}}$ is a multiple of $p^{\frac{\nu_{p}(D)}{2}}$. Then by (4.19), (4.18) is true for each $1 \leq i \leq \nu_{p}(D)$. For $\nu_{p}(D) \leq i \leq l$, we can deduce from $f(n) \equiv 0\left(\bmod p^{i}\right)$ that $\nu_{p}(2 a n+b) \geq \nu_{p}(D) / 2$, which implies that

$\nu_{p}\left(f\left(n \pm m p^{l-\frac{\nu_{p}(D)}{2}}\right)\right) \geq \min \left\{i, \nu_{p}(D) / 2+l-\nu_{p}(D) / 2+\nu_{p}(m), 2 l-\nu_{p}(D)+\nu_{p}(m)\right\} \geq i$.

Conversely, if $\nu_{p}\left(f\left(n+m p^{l-\frac{\nu_{p}(D)}{2}}\right)\right) \geq i$, then replacing $n$ with $n+m p^{l-\frac{\nu_{p}(D)}{2}}$ in (4.20), we get $\nu_{p}(f(n))=\nu_{p}\left(f\left(n+m p^{l-\frac{\nu_{p}(D)}{2}}-m p^{l-\frac{\nu_{p}(D)}{2}}\right)\right) \geq i$. Hence (4.18) is proved.

If either $e \leq \nu_{p}(D)$ and $\nu_{p}(k+1) \geq\lceil e / 2\rceil$, or $e>\nu_{p}(D)$ and $\nu_{p}(k+1) \geq e-\nu_{p}(D) / 2$, then either $p^{\lceil e / 2\rceil} \mid(k+1)$, or $p^{e-\nu_{p}(D) / 2} \mid(k+1)$. It then follows immediately from (4.3) and (4.18) with $l=e$ that for each $1 \leq i \leq e, \nu_{p}(f(n)) \geq i$ if and only if $\nu_{p}(f(n+k+1)) \geq$ $i$. But the distinct terms of the sets $S_{k, f}(n)$ and $S_{k, f}(n+1)$ are $f(n)$ and $f(n+k+1)$. Thus the number of terms divisible by $p^{i}$ in $S_{k, f}(n)$ is equal to that in $S_{k, f}(n+1)$ for any $i \in\{1, \ldots, e\}$. Thus we have $h_{p, i}(n+1)=h_{p, i}(n)$ for each $i \in\{1, \ldots, e\}$, and so by (4.17), $g_{p, k, f}(n+1)=g_{p, k, f}(n)$ for any positive integer $n$. Hence $P_{p, k, f}=1$. So Lemma 4.4 is true if either $e \leq \nu_{p}(D)$ and $\nu_{p}(k+1) \geq\lceil e / 2\rceil$, or $e>\nu_{p}(D)$ and $\nu_{p}(k+1) \geq e-\nu_{p}(D) / 2$.

Now let $e \leq \nu_{p}(D)$ and $\nu_{p}(k+1)<\lceil e / 2\rceil$. Taking $m=1$ in (4.3), we have that for any given $1 \leq i \leq e$ and for any $0 \leq j \leq k, \nu_{p}(f(n+j)) \geq i$ if and only if $\nu_{p}\left(f\left(n+j+p^{\lceil e / 2\rceil}\right) \geq\right.$ $i$. In other words, the number of terms divisible by $p^{i}$ in $S_{k, f}(n)$ is equal to that in $S_{k, f}\left(n+p^{\lceil e / 2\rceil}\right)$ for any $i \in\{1, \ldots, e\}$. It infers that $h_{p, i}\left(n+p^{\lceil e / 2\rceil}\right)=h_{p, i}(n)$ for each $i \in\{1, \ldots, e\}$. Thus by (4.17) $g_{p, k, f}\left(n+p^{\lceil e / 2\rceil}\right)=g_{p, k, f}(n)$ for any positive integer $n$, and so $p^{\lceil e / 2\rceil}$ is a period of $g_{p, k, f}$. But Lemma 4.1 implies that there is a positive integer $n_{0}$ such that $g_{p, k, f}\left(n_{0}+p^{\lceil e / 2\rceil-1}\right) \neq g_{p, k, f}\left(n_{0}\right)$. Therefore $p^{\lceil e / 2\rceil-1}$ is not the period of 
$g_{p, k, f}$. Thus $p^{\lceil e / 2\rceil}$ is the smallest period of $g_{p, k, f}$ as required. Thus Lemma 4.4 is true if $e \leq \nu_{p}(D)$ and $\nu_{p}(k+1)<\lceil e / 2\rceil$.

We only need to deal with the remaining case: $e>\nu_{p}(D)$ and $\nu_{p}(k+1)<e-\nu_{p}(D) / 2$ which will be done in what follows. First, from (4.18) with $l=e$ and $m=1$, it follows that for any given $1 \leq i \leq e$ and for any $0 \leq j \leq k, \nu_{p}(f(n+j)) \geq i$ if and only if $\nu_{p}\left(f\left(n+j+p^{e-\nu_{p}(D) / 2}\right)\right) \geq i$. Namely, the number of terms divisible by $p^{i}$ in $S_{k, f}(n)$ is equal to that in $S_{k, f}\left(n+p^{e-\nu_{p}(D) / 2}\right)$ for each $i \in\{1, \ldots, e\}$. Hence $g_{p, k, f}\left(n+p^{e-\nu_{p}(D) / 2}\right)=$ $g_{p, k, f}(n)$ for any positive integer $n$ by (4.17). Thus $p^{e-\nu_{p}(D) / 2}$ is a period of $g_{p, k, f}$.

By Lemma 3.3 (iii), we know that the congruence $f(x) \equiv 0\left(\bmod p^{e}\right)$ has exactly two solutions in the interval $\left[1, p^{e-\frac{\nu_{p}(D)}{2}}\right]$. It follows that $d_{p^{e}} \leq\left(p^{e-\nu_{p}(D) / 2}-1\right) / 2$. Therefore, we can find a positive integer $u_{0}$ with $1 \leq u_{0} \leq \frac{p+1}{2}$ such that $\left(u_{0}-1\right) p^{e-\nu_{p}(D) / 2-1} \leq$ $d_{p^{e}}<u_{0} p^{e-\nu_{p}(D) / 2-1}$. To prove that $p^{e-\nu_{p}(D) / 2}$ is the smallest period of $g_{p, k, f}$, it suffices to prove that $u_{0} p^{e-\frac{\nu_{p}(D)}{2}-1}$ is not a period of $g_{p, k, f}$. For this purpose, we define the arithmetic function $\Delta$ for any positive integer $n$ by

$$
\Delta(n):=g_{p, k, f}\left(n+u_{0} p^{e-\nu_{p}(D) / 2-1}\right)-g_{p, k, f}(n) .
$$

Since $e>\nu_{p}(D)$, we have $e-1 \geq \nu_{p}(D)$. Picking $l=e-1$ and $m=u_{0}$ in (4.18), we get that for any given $1 \leq i \leq e-1$ and for any $0 \leq j \leq k, \nu_{p}\left(f\left(n+j+u_{0} p^{e-\nu_{p}(D) / 2-1}\right)\right) \geq i$ if and only if $\nu_{p}(f(n+j)) \geq i$. Hence the number of terms divisible by $p^{i}$ in $S_{k, f}(n)$ is equal to that in $S_{k, f}\left(n+u_{0} p^{e-\nu_{p}(D) / 2-1}\right)$, i.e., $h_{p, i}\left(n+u_{0} p^{e-\nu_{p}(D) / 2-1}\right)=h_{p, i}(n)$ for each $1 \leq i \leq e-1$. So by (4.17) and (4.21), we get

$$
\Delta(n)=h_{p, e}\left(n+u_{0} p^{e-\nu_{p}(D) / 2-1}\right)-h_{p, e}(n) .
$$

Define the two sets $\mathcal{A}_{1}(n):=\left\{f(n), \ldots, f\left(n+d_{p^{e}}\right), \ldots, f\left(n+u_{0} p^{e-\nu_{p}(D) / 2-1}-1\right)\right\}$ and $\mathcal{A}_{2}(n):=\left\{f(n+k+1), \ldots, f\left(n+k+u_{0} p^{e-\nu_{p}(D) / 2-1}\right)\right\}$. Evidently, $S_{k, f}\left(n+u_{0} p^{e-\nu_{p}(D) / 2-1}\right) \subseteq$ $\mathcal{A}_{2}(n)$ if $k<u_{0} p^{e-\nu_{p}(D) / 2-1}$. If $k \geq u_{0} p^{e-\nu_{p}(D) / 2-1}$, then we have the following disjoint unions: $S_{k, f}(n)=\mathcal{A}_{1}(n) \bigcup\left\{f\left(n+u_{0} p^{e-\nu_{p}(D) / 2-1}\right), \ldots, f(n+k)\right\}$ and $S_{k, f}(n+$ $\left.u_{0} p^{e-\nu_{p}(D) / 2-1}\right)=\left\{f\left(n+u_{0} p^{e-\nu_{p}(D) / 2-1}\right), \ldots, f(n+k)\right\} \cup \mathcal{A}_{2}(n)$. Claim that there is a positive integer $n_{0}$ such that the set $S_{k, f}\left(n_{0}\right)$ contains exactly two terms divisible by $p^{e}$ if $k<u_{0} p^{e-\nu_{p}(D) / 2-1}$, while the set $\mathcal{A}_{1}\left(n_{0}\right)$ holds exactly two terms divisible by $p^{e}$ and the set $\mathcal{A}_{2}\left(n_{0}\right)$ has at most one term divisible by $p^{e}$.

Suppose that the claim is true. If $k<u_{0} p^{e-\nu_{p}(D) / 2-1}$, then it follows from the claim that $h_{p, e}\left(n_{0}+u_{0} p^{e-\nu_{p}(D) / 2-1}\right)=\max \left(0, \#\left\{m \in S_{k, f}\left(n_{0}+u_{0} p^{e-\nu_{p}(D) / 2-1}\right)\right.\right.$ : $\left.\left.p^{e} \mid m\right\}-1\right)=0$ and $h_{p, e}\left(n_{0}\right)=\#\left\{m \in S_{k, f}\left(n_{0}\right): p^{e} \mid m\right\}-1=1$. Hence by (4.22), we get $\Delta\left(n_{0}\right)=-1$. If $k \geq u_{0} p^{e-\nu_{p}(D) / 2-1}$, then we derive from the claim that $h_{p, e}\left(n_{0}+u_{0} p^{e-\nu_{p}(D) / 2-1}\right) \leq \#\left\{u_{0} p^{e-\nu_{p}(D) / 2-1} \leq j \leq k: p^{e} \mid f\left(n_{0}+j\right)\right\}$ and $h_{p, e}\left(n_{0}\right)=\#\left\{u_{0} p^{e-\nu_{p}(D) / 2-1} \leq j \leq k: p^{e} \mid f\left(n_{0}+j\right)\right\}+1$. It follows from (4.22) that $\Delta\left(n_{0}\right) \leq-1$. Therefore $u_{0} p^{e-\nu_{p}(D) / 2-1}$ is not a period of $g_{p, k, f}$. Thus Lemma 4.4 is true if $e>\nu_{p}(D)$ and $\nu_{p}(k+1)<e-\nu_{p}(D) / 2$. It remains to prove that the claim is true.

First note that by Lemma 3.3 (iii), there are exactly two terms divisible by $p^{e}$ in any consecutive $p^{e-\nu_{p}(D) / 2}$ terms of the quadratic progression $\{f(n)\}_{n=1}^{\infty}$. Since $\nu_{p}(k+1)<$ $e-\nu_{p}(D) / 2$, we can find some integer $r$ with $1 \leq r \leq p^{e-\nu_{p}(D) / 2}-1$ such that $k+1 \equiv r$ $\left(\bmod p^{e-\nu_{p}(D) / 2}\right)$. We divide the proof of the claim into the following two cases.

Case 1. $r \in\left[1, u_{0} p^{e-\frac{\nu_{p}(D)}{2}-1}\right]$ with $u_{0} \in\left[1, \frac{p-1}{2}\right]$, or $r \in\left[1,(p-1) p^{e-\nu_{p}(D) / 2-1} / 2\right] \cup$ $\left(d_{p^{e}},(p+1) p^{e-\nu_{p}(D) / 2-1} / 2\right]$ with $u_{0}=\frac{p+1}{2}$. By Lemma 3.6 we can choose a positive integer $n_{0}$ such that $\nu_{p}\left(f\left(n_{0}\right)\right) \geq e$ and $\nu_{p}\left(f\left(n_{0}+d_{p^{e}}\right)\right) \geq e$. By Lemma 3.3 (iii), we know that the terms divisible by $p^{e}$ in the quadratic progression $\left\{f\left(n_{0}+j\right)\right\}_{j \in \mathbb{N}}$ must 
be of the form $f\left(n_{0}+t_{1} p^{e-\nu_{p}(D) / 2}\right)$ or $f\left(n_{0}+d_{p^{e}}+t_{2} p^{e-\nu_{p}(D) / 2}\right), t_{1}, t_{2} \in \mathbb{N}$. Since $\left|\mathcal{A}_{1}\left(n_{0}\right)\right|=u_{0} p^{e-\nu_{p}(D)-1}<p^{e-\nu_{p}(D) / 2}$ and $d_{p^{e}}<u_{0} p^{e-\nu_{p}(D) / 2-1}, f\left(n_{0}\right)$ and $f\left(n_{0}+d_{p^{e}}\right)$ are the exactly two terms divisible by $p^{e}$ in $\mathcal{A}_{1}\left(n_{0}\right)$. On the other hand, since $k \geq$ $d_{p^{e}}$ and $\left|S_{k, f}\left(n_{0}\right)\right|=k+1 \leq u_{0} p^{e-\nu_{p}(D) / 2-1}<p^{e-\nu_{p}(D) / 2}, f\left(n_{0}\right)$ and $f\left(n_{0}+d_{p^{e}}\right)$ are exactly the two terms divisible by $p^{e}$ in $S_{k, f}\left(n_{0}\right)$ if $k<u_{0} p^{e-\nu_{p}(D) / 2-1}$. Namely, $\mathcal{A}_{1}\left(n_{0}\right)$ holds exactly two terms divisible by $p^{e}$ and $S_{k, f}\left(n_{0}\right)$ contains exactly two terms divisible by $p^{e}$ if $k<u_{0} p^{e-\nu_{p}(D) / 2-1}$. Now we show that $\mathcal{A}_{2}\left(n_{0}\right)$ has at most one term divisible by $p^{e}$. Since $\left|\mathcal{A}_{2}\left(n_{0}\right)\right|=u_{0} p^{e-\nu_{p}(D) / 2-1}<p^{e-\nu_{p}(D) / 2}$, there is at most one term of the form $f\left(n_{0}+t_{1} p^{e-\nu_{p}(D) / 2}\right)$ and there is at most one term of the form $f\left(n_{0}+d_{p^{e}}+t_{2} p^{e-\nu_{p}(D) / 2}\right)$ in the set $\mathcal{A}_{2}\left(n_{0}\right)$ with $t_{1}, t_{2} \in \mathbb{N}$. Therefore, we only need to show that either there is no term of the form $f\left(n_{0}+t_{1} p^{e-\nu_{p}(D) / 2}\right)$, or there is no term of the form $f\left(n_{0}+d_{p^{e}}+t_{2} p^{e-\nu_{p}(D) / 2}\right)$ in the set $\mathcal{A}_{2}\left(n_{0}\right)$, where $t_{1}, t_{2} \in \mathbb{N}$, which will be done in the following.

If $r \in\left[1, u_{0} p^{e-\frac{\nu_{p}(D)}{2}-1}\right]$ with $u_{0} \in\left[1, \frac{p-1}{2}\right]$, we have that for all $1 \leq j \leq u_{0} p^{e-\nu_{p}(D) / 2-1}$, $k+j \equiv r+j-1 \not \equiv 0\left(\bmod p^{e-\nu_{p}(D) / 2}\right)$ since $1 \leq r+j-1 \leq 2 u_{0} p^{e-\nu_{p}(D) / 2-1}-1<$ $p^{e-\nu_{p}(D) / 2}-1$. Hence there is no term of the form $f\left(n_{0}+t_{1} p^{e-\nu_{p}(D) / 2}\right)$ in the set $\mathcal{A}_{2}\left(n_{0}\right)$ with $t_{1} \in \mathbb{N}$.

If $r \in\left[1,(p-1) p^{e-\nu_{p}(D) / 2-1} / 2\right] \cup\left(d_{p^{e}},(p+1) p^{e-\nu_{p}(D) / 2-1} / 2\right]$ with $u_{0}=\frac{p+1}{2}$, we have for all $1 \leq j \leq u_{0} p^{e-\nu_{p}(D) / 2-1}$ that $1 \leq r+j-1 \leq(p-1) p^{e-\nu_{p}(D) / 2-1} / 2+$ $u_{0} p^{e-\nu_{p}(D) / 2-1}-1=p^{e-\nu_{p}(D) / 2}-1$ if $r \in\left[1,(p-1) p^{e-\nu_{p}(D) / 2-1} / 2\right]$ and that $d_{p^{e}}<$ $r+j-1 \leq(p+1) p^{e-\nu_{p}(D) / 2-1}-1 \leq p^{e-\nu_{p}(D) / 2}+d_{p^{e}}-1$ if $r \in\left(d_{p^{e}},(p+1) p^{e-\nu_{p}(D) / 2-1} / 2\right]$ since $d_{p^{e}} \geq\left(u_{0}-1\right) p^{e-\nu_{p}(D) / 2-1}=\frac{p-1}{2} p^{e-\nu_{p}(D) / 2-1} \geq p^{e-\nu_{p}(D) / 2-1}$. That is, for all $1 \leq j \leq u_{0} p^{e-\nu_{p}(D) / 2-1}$, we have $k+j \equiv r+j-1 \not \equiv 0\left(\bmod p^{e-\nu_{p}(D) / 2}\right)$ if $r \in$ $\left[1,(p-1) p^{e-\nu_{p}(D) / 2-1} / 2\right]$ and $k+j \equiv r+j-1 \not \equiv d_{p^{e}}\left(\bmod p^{e-\nu_{p}(D) / 2}\right)$ if $r \in\left(d_{p^{e}},(p+\right.$ 1) $\left.p^{e-\nu_{p}(D) / 2-1} / 2\right]$. Therefore, there is no term of the form $f\left(n_{0}+t_{1} p^{e-\nu_{p}(D)}\right)$ in $\mathcal{A}_{2}\left(n_{0}\right)$ if $r \in\left[1,(p-1) p^{e-\nu_{p}(D) / 2-1} / 2\right]$ with $u_{0}=\frac{p+1}{2}$, and there is no term of the form $f\left(n_{0}+\right.$ $\left.d_{p^{e}}+t_{2} p^{e-\nu_{p}(D) / 2-1}\right)$ in $\mathcal{A}_{2}\left(n_{0}\right)$ if $r \in\left(d_{p^{e}},(p+1) p^{e-\nu_{p}(D) / 2-1} / 2\right]$ with $u_{0}=\frac{p+1}{2}$, where $t_{1}, t_{2} \in \mathbb{N}$. So the claim is proved for Case 1 .

Case 2. $r \in\left(u_{0} p^{e-\frac{\nu_{p}(D)}{2}-1}, p^{e-\nu_{p}(D) / 2}-1\right]$ with $u_{0} \in\left[1, \frac{p-1}{2}\right]$, or $r \in\left((p+1) p^{e-\frac{\nu_{p}(D)}{2}-1} / 2\right.$, $\left.p^{e-\nu_{p}(D) / 2}-1\right] \cup\left((p-1) p^{e-\nu_{p}(D) / 2-1} / 2, d_{p^{e}}\right]$ with $u_{0}=\frac{p+1}{2}$. Then by Lemma 3.6, we can select a positive integer $n_{0}$ such that $\nu_{p}\left(f\left(n_{0}+u_{0} p^{e-\nu_{p}(D) / 2-1}-1\right)\right)=e$ and $\nu_{p}\left(f\left(n_{0}+u_{0} p^{e-\nu_{p}(D) / 2-1}-1-d_{p^{e}}\right)\right) \geq e$. Now by Lemma 3.3 (iii), the terms divisible by $p^{e}$ in the quadratic progression $\left\{f\left(n_{0}+j\right)\right\}_{j \in \mathbb{N}}$ are of the form

$$
f\left(n_{0}+u_{0} p^{e-\nu_{p}(D) / 2-1}-1+t_{1} p^{e-\nu_{p}(D) / 2}\right)
$$

or

$$
f\left(n_{0}+u_{0} p^{e-\nu_{p}(D) / 2-1}-1-d_{p^{e}}+t_{2} p^{e-\nu_{p}(D) / 2}\right),
$$

where $t_{1}, t_{2} \in \mathbb{N}$.

Since $k+1 \equiv r\left(\bmod p^{e-\nu_{p}(D) / 2}\right)$, one may let $k=r-1+t p^{e-\nu_{p}(D)}$ for some integer $t \geq 0$. It follows that $k \geq r-1 \geq u_{0} p^{e-\nu_{p}(D) / 2-1}$ if $r \in\left(u_{0} p^{e-\frac{\nu_{p}(D)}{2}-1}, p^{e-\nu_{p}(D) / 2}-1\right]$ with $u_{0} \in\left[1, \frac{p-1}{2}\right]$, or $r \in\left((p+1) p^{e-\frac{\nu_{p}(D)}{2}-1} / 2, p^{e-\nu_{p}(D) / 2}-1\right]$ with $u_{0}=\frac{p+1}{2}$. If $r \in\left((p-1) p^{e-\nu_{p}(D) / 2-1} / 2, d_{p^{e}}\right]$ with $u_{0}=\frac{p+1}{2}$, then it follows from $k \geq d_{p^{e}}$ and $r \leq d_{p^{e}}$ that $t \geq 1$. Thus $k \geq r-1+p^{e-\nu_{p}(D)} \geq u_{0} p^{e-\nu_{p}(D) / 2-1}$. That is, we always have $k \geq u_{0} p^{e-\nu_{p}(D) / 2-1}$ in Case 2. Hence to finish the proof of the claim for Case 2, we only need to treat with the two sets $\mathcal{A}_{1}\left(n_{0}\right)$ and $\mathcal{A}_{2}\left(n_{0}\right)$. 
Evidently, $u_{0} p^{e-\nu_{p}(D) / 2-1}-1-d_{p^{e}} \geq 0$ since $d_{p^{e}}<u_{0} p^{e-\nu_{p}(D) / 2-1}$. Again using the fact $\left|\mathcal{A}_{1}\left(n_{0}\right)\right|=u_{0} p^{e-\nu_{p}(D)-1}<p^{e-\nu_{p}(D) / 2}$, we know that $f\left(n_{0}+u_{0} p^{e-\nu_{p}(D) / 2-1}-1\right)$ and $f\left(n_{0}+u_{0} p^{e-\nu_{p}(D) / 2-1}-1-d_{p^{e}}\right)$ are the exactly two terms divisible by $p^{e}$ in $\mathcal{A}_{1}\left(n_{0}\right)$.

Since $\left|\mathcal{A}_{2}\left(n_{0}\right)\right|=u_{0} p^{e-\nu_{p}(D) / 2-1}$, to show that $\mathcal{A}_{2}\left(n_{0}\right)$ has at most one term divisible by $p^{e}$, it is enough to show that either there is no term of the form (4.23), or there is no term of the form (4.24) in the set $\mathcal{A}_{2}\left(n_{0}\right)$, where $t_{1}, t_{2} \in \mathbb{N}$.

If either $r \in\left(u_{0} p^{e-\frac{\nu_{p}(D)}{2}-1}, p^{e-\nu_{p}(D) / 2}-1\right]$ with $u_{0} \in[1,(p-1) / 2]$, or $r \in((p+$ 1) $\left.p^{e-\frac{\nu_{p}(D)}{2}-1} / 2, p^{e-\nu_{p}(D) / 2}-1\right]$ with $u_{0}=\frac{p+1}{2}$, then for all $1 \leq j \leq u_{0} p^{e-\nu_{p}(D) / 2-1}$, we have $u_{0} p^{e-\nu_{p}(D) / 2-1}<r+j-1<p^{e-\nu_{p}(D) / 2}+u_{0} p^{e-\nu_{p}(D) / 2-1}-1$, which implies that $k+j \equiv r+j-1 \not \equiv u_{0} p^{e-\nu_{p}(D) / 2-1}-1\left(\bmod p^{e-\nu_{p}(D) / 2}\right)$. Hence there is no term of the form (4.23) with $t_{1} \in \mathbb{N}$ in $\mathcal{A}_{2}\left(n_{0}\right)$.

If $r \in\left((p-1) p^{e-\nu_{p}(D) / 2-1} / 2, d_{p^{e}}\right]$ with $u_{0}=\frac{p+1}{2}$, then

$$
\begin{aligned}
\frac{p-1}{2} p^{e-\nu_{p}(D) / 2-1} & <r+j-1 \leq d_{p^{e}}+\frac{p+1}{2} p^{e-\nu_{p}(D) / 2-1}-1 \\
& <p^{e-\nu_{p}(D) / 2}+\frac{p+1}{2} p^{e-\nu_{p}(D) / 2-1}-1-d_{p^{e}}
\end{aligned}
$$

for all $1 \leq j \leq u_{0} p^{e-\nu_{p}(D) / 2-1}$ since $d_{p^{e}} \leq \frac{p^{e-\nu_{p}(D) / 2}-1}{2}$. However,

$$
\begin{aligned}
\frac{p+1}{2} p^{e-\nu_{p}(D) / 2-1}-1-d_{p^{e}} & <\frac{p+1}{2} p^{e-\nu_{p}(D) / 2-1}-1-\frac{p-1}{2} p^{e-\nu_{p}(D) / 2-1} \\
& =p^{e-\nu_{p}(D) / 2-1}-1<\frac{p-1}{2} p^{e-\nu_{p}(D) / 2-1} .
\end{aligned}
$$

It then follows that $k+j \equiv r+j-1 \not \equiv \frac{p+1}{2} p^{e-\nu_{p}(D) / 2-1}-1-d_{p^{e}}\left(\bmod p^{e-\nu_{p}(D) / 2}\right)$ for all $1 \leq j \leq u_{0} p^{e-\nu_{p}(D) / 2-1}$. So there is no term of the form (4.24) with $t_{2} \in \mathbb{N}$ in the set $\mathcal{A}_{2}\left(n_{0}\right)$. So the claim is true in Case 2.

The proof of Lemma 4.4 is complete.

From Lemmas 4.3 and 4.4 , we see that $\nu_{p}\left(P_{p, k, f}\right)$ depends on some nonnegative integer $e$ satisfying $d_{p^{e}} \leq k<d_{p^{e+1}}$. In other words, we still don't get the explicit value of $P_{p, k, f}$. Thus, to determine the exact value of $P_{p, k, f}$ for those primes $p \nmid a$, we need to transform the information on $e$ into explicit information depending on $k$ and $f$. We have the following results.

Lemma 4.5. Let $a$ be odd and $\mathcal{K}_{f}$ be nonempty. Then for any $k \in \mathcal{K}_{f}$, we have

$P_{2, k, f}=\left\{\begin{aligned} 2^{\nu_{2}\left(B_{k}\right)-2 \nu_{2}\left(L_{k}\right)}, & \text { if } k<2^{\left\lfloor\frac{\nu_{2}(D)}{2}\right\rfloor} \text { and } \nu_{2}(k+1)<\nu_{2}\left(L_{k}\right), \\ 2^{\left\lfloor\frac{\nu_{2}(D)}{2}\right\rfloor}, & \text { if } k \geq 2^{\left\lfloor\frac{\nu_{2}(D)}{2}\right\rfloor}, D_{4} \neq \equiv 1 \quad(\bmod 8) \text { and } \nu_{2}(k+1)<\left\lfloor\frac{\nu_{2}(D)}{2}\right\rfloor, \\ 2^{\nu_{2}\left(B_{k}\right)-\nu_{2}(D)-1}, & \text { if } k \geq 2^{\left\lfloor\frac{\nu_{2}(D)}{2}\right\rfloor} \text { and } D_{4} \equiv 1 \quad(\bmod 8), \\ 1, & \text { otherwise. }\end{aligned}\right.$

Proof. Since $\mathcal{K}_{f}$ is nonempty and $k \in \mathcal{K}_{f}$, by Lemma 3.12 , there is a unique nonnegative integer $e$ such that $d_{2^{e}} \leq k<d_{2^{e+1}}$. Consider the following three cases.

Case 1. $k<2^{\left\lfloor\frac{\nu_{2}(D)}{2}\right\rfloor}$. Since $2 \nmid a$, we have $\nu_{2}\left(a^{2} i^{2}\right)=2 \nu_{2}(i) \leq 2\left(\left\lfloor\frac{\nu_{2}(D)}{2}\right\rfloor-1\right) \leq$ $\nu_{2}(D)-2<\nu_{2}(D)$ and so $\nu_{2}\left(a^{2} i^{2}-D\right)=2 \nu_{2}(i)$ for any integer $i$ with $1 \leq i \leq k$. Hence

$$
\max _{1 \leq i \leq k}\left\{\nu_{2}\left(a^{2} i^{2}-D\right)\right\}=\max _{1 \leq i \leq k}\left\{2 \nu_{2}(i)\right\}=2 \nu_{2}\left(L_{k}\right)
$$

But 
$\nu_{2}\left(B_{k}\right)=\max _{1 \leq i \leq k}\left\{\nu_{2}\left(i\left(a^{2} i^{2}-D\right)\right)\right\}=\max _{1 \leq i \leq k}\left\{\nu_{2}(i)+\nu_{2}\left(a^{2} i^{2}-D\right)\right\}=\max _{1 \leq i \leq k}\left\{3 \nu_{2}(i)\right\}=3 \nu_{2}\left(L_{k}\right)$.

By Lemma 3.9 (i), we have $d_{2^{2\left\lfloor\frac{\nu_{2}(D)}{2}\right\rfloor-1}}=2^{\left\lfloor\frac{\nu_{2}(D)}{2}\right\rfloor}$. Thus by Lemma 3.7 and $d_{2^{e}} \leq k<$ $2^{\left\lfloor\frac{\nu_{2}(D)}{2}\right\rfloor}$, we have $e<2\left\lfloor\frac{\nu_{2}(D)}{2}\right\rfloor-1$. Notice that by part (i) of Lemma 3.9, $d_{2^{e}}$ (resp. $\left.d_{2^{e+1}}\right)$ is the smallest positive root of the congruence $a^{2} x^{2}-D \equiv 0\left(\bmod 2^{e}\right)($ resp. $\left.a^{2} x^{2}-D \equiv 0\left(\bmod 2^{e+1}\right)\right)$. Hence $2^{e+1} \nmid\left(a^{2} l^{2}-D\right)$ for all positive integers $l<d_{2^{e+1}}$. But $d_{2^{e}} \leq k<d_{2^{e+1}}$. Thus $\max _{1 \leq i \leq k}\left\{\nu_{2}\left(a^{2} i^{2}-D\right)\right\} \geq e$ and $\max _{1 \leq i \leq k}\left\{\nu_{2}\left(a^{2} i^{2}-D\right)\right\}<e+1$. Then by (4.25), $e=\max _{1 \leq i \leq k}\left\{\nu_{2}\left(a^{2} i^{2}-D\right)\right\}=2 \nu_{2}\left(L_{k}\right)$. Therefore, by Lemma 4.3 (i) and (4.26), we get that $P_{2, k, f}=2^{\lceil e / 2\rceil}=2^{\nu_{2}\left(L_{k}\right)}=2^{\nu_{2}\left(B_{k}\right)-2 \nu_{2}\left(L_{k}\right)}$ if $k<2^{\left\lfloor\frac{\nu_{2}(D)}{2}\right\rfloor}$ and $\nu_{2}(k+1)<\nu_{2}\left(L_{k}\right)$, and $P_{2, k, f}=1$ if $k<2^{\left\lfloor\frac{\nu_{2}(D)}{2}\right\rfloor}$ and $\nu_{2}(k+1) \geq \nu_{2}\left(L_{k}\right)$. Thus Lemma 4.5 is true in this case.

Case 2. $k \geq 2^{\left\lfloor\frac{\nu_{2}(D)}{2}\right\rfloor}$ and $D_{4} \neq \equiv 1(\bmod 8)$. By parts (i) and (ii) of Lemma 3.9, one knows that $d_{2^{2\lfloor}\left\lfloor\frac{\nu_{2}(D)}{2}\right\rfloor-1}=2^{\left\lfloor\frac{\nu_{2}(D)}{2}\right\rfloor}$ and $d_{2^{2\lfloor}\left\lfloor\frac{\nu_{2}(D)}{2}\right\rfloor}=\infty$ if $D_{4} \not \equiv 1(\bmod 4)$, and $d_{2^{\nu_{2}(D)}}=$ $2^{\frac{\nu_{2}(D)}{2}}$ and $d_{2^{\nu_{2}(D)+1}}=\infty$ if $D_{4} \equiv 5(\bmod 8)$. It then follows from $d_{2^{e}} \leq k<d_{2^{e+1}}$ and $k \geq 2^{\left\lfloor\frac{\nu_{2}(D)}{2}\right\rfloor}$ that

$$
e=\left\{\begin{array}{rll}
2\left\lfloor\frac{\nu_{2}(D)}{2}\right\rfloor-1, & \text { if } D_{4} \not \equiv 1 & (\bmod 4), \\
\nu_{2}(D), & \text { if } D_{4} \equiv 5 \quad(\bmod 8) .
\end{array}\right.
$$

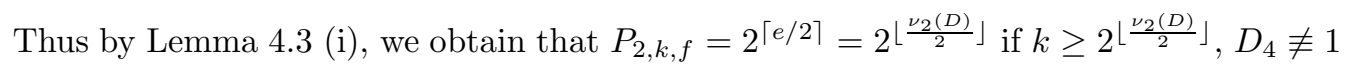
$(\bmod 8)$ and $\nu_{2}(k+1)<\left\lfloor\frac{\nu_{2}(D)}{2}\right\rfloor$, and $P_{2, k, f}=1$ if $k \geq 2^{\left\lfloor\frac{\nu_{2}(D)}{2}\right\rfloor}, D_{4} \not \equiv 1(\bmod 8)$ and $\nu_{2}(k+1) \geq\left\lfloor\frac{\nu_{2}(D)}{2}\right\rfloor$. Lemma 4.5 is true in Case 2 .

Case 3. $k \geq 2^{\left\lfloor\frac{\nu_{2}(D)}{2}\right\rfloor}$ and $D_{4} \equiv 1(\bmod 8)$. Then $\nu_{2}(D)$ is even and $\nu_{2}\left(L_{k}\right) \geq \frac{\nu_{2}(D)}{2}$. Since $2^{\frac{\nu_{2}(D)}{2}}$ is equal to the smallest positive root of the congruence $a^{2} x^{2}-D \equiv 0$ $\left(\bmod 2^{\nu_{2}(D)+3}\right)$, we derive from Lemma 3.9 (iii) that $d_{2^{\nu_{2}(D)+2}}=2^{\frac{\nu_{2}(D)}{2}}$. Hence from Lemma 3.7 and $d_{2^{e}} \leq k<d_{2^{e+1}}$, we can derive that $e \geq \nu_{2}(D)+2$. So Lemma 3.13 (i) gives that $e=\max _{1 \leq i \leq k}\left\{\nu_{2}\left(a^{2} i^{2}-D\right)\right\}-1$. It follows from Lemma 4.3 (ii) that $\nu_{2}\left(P_{2, k, f}\right)=e-\frac{\nu_{2}(D)}{2}=\max _{1 \leq i \leq k}\left\{\nu_{2}\left(a^{2} i^{2}-D\right)\right\}-\frac{\nu_{2}(D)}{2}-1$. Therefore, to show that $\nu_{2}\left(P_{2, k, f}\right)=\nu_{2}\left(B_{k}\right)-\nu_{2}(D)-1$, it suffices to prove that the following is true:

$$
\nu_{2}\left(B_{k}\right)=\max _{1 \leq i \leq k}\left\{\nu_{2}\left(a^{2} i^{2}-D\right)\right\}+\frac{\nu_{2}(D)}{2},
$$

which will be done in what follows. Let $i$ be an integer such that $1 \leq i \leq k$. Then we have that

$$
\nu_{2}\left(i\left(a^{2} i^{2}-D\right)\right)=\nu_{2}(i)+\min \left(2 \nu_{2}(i), \nu_{2}(D)\right)<\nu_{2}\left(L_{k}\right)+\nu_{2}(D)
$$

if $\nu_{2}(i)<\frac{\nu_{2}(D)}{2}$, and that

$$
\nu_{2}\left(i\left(a^{2} i^{2}-D\right)\right)=\nu_{2}(i)+\nu_{2}\left(a^{2} i^{2}-D\right)=\frac{\nu_{2}(D)}{2}+\nu_{2}\left(a^{2} i^{2}-D\right)
$$

if $\nu_{2}(i)=\frac{\nu_{2}(D)}{2}$, and that

$$
\nu_{2}\left(i\left(a^{2} i^{2}-D\right)\right)=\nu_{2}(i)+\min \left\{\nu_{2}\left(a^{2} i^{2}\right), \nu_{2}(D)\right\}=\nu_{2}(i)+\nu_{2}(D) \leq \nu_{2}\left(L_{k}\right)+\nu_{2}(D)
$$


if $\nu_{2}(i)>\frac{\nu_{2}(D)}{2}$. Now we claim that

$$
\max _{\substack{1 \leq i \leq k \\ \nu_{2}(i)=\nu_{2}(D) / 2}}\left\{\nu_{2}\left(a^{2} i^{2}-D\right)\right\} \geq \nu_{2}\left(L_{k}\right)+\frac{\nu_{2}(D)}{2}+1 .
$$

This is equivalent to showing that there is an integer $i_{0} \in[1, k]$ with $\nu_{2}\left(i_{0}\right)=\frac{\nu_{2}(D)}{2}$ such that $\left.\nu_{2}\left(a^{2} i_{0}^{2}-D\right)\right\} \geq \nu_{2}\left(L_{k}\right)+\frac{\nu_{2}(D)}{2}+1$.

If $\nu_{2}\left(L_{k}\right) \leq \frac{\nu_{2}(D)}{2}+2$ and $D_{4} \equiv 1(\bmod 8)$, then pick $i_{0}=2^{\frac{\nu_{2}(D)}{2}} \in[1, k]$. Since $2 \nmid a$, we have $\nu_{2}\left(a^{2}\left(2^{\frac{\nu_{2}(D)}{2}}\right)^{2}-D\right)=\nu_{2}(D)+\nu_{2}\left(a^{2}-D_{4}\right) \geq \nu_{2}(D)+3 \geq \nu_{2}\left(L_{k}\right)+\frac{\nu_{2}(D)}{2}+1$.

If $\nu_{2}\left(L_{k}\right)>\frac{\nu_{2}(D)}{2}+2$ and $D_{4} \equiv 1(\bmod 8)$, then $\nu_{2}\left(L_{k}\right)+\frac{\nu_{2}(D)}{2}+1>\nu_{2}(D)+3$. Since the discriminant of $a^{2} x^{2}-D$ is $4 a^{2} D$ and $\nu_{2}\left(4 a^{2} D\right)=\nu_{2}(D)+2$, then Lemma 3.2 (iv) applied to the congruence $a^{2} x^{2}-D \equiv 0\left(\bmod 2^{\nu_{2}\left(L_{k}\right)+\frac{\nu_{2}(D)}{2}+1}\right)$, we can find an integer $i_{0} \in\left[1,2^{\nu_{2}\left(L_{k}\right)}\right] \subseteq[1, k]$ satisfying that $\nu_{2}\left(i_{0}\right)=\frac{\nu_{2}(D)}{2}$ and $a^{2} i_{0}^{2}-D \equiv 0$ $\left(\bmod 2^{\nu_{2}\left(L_{k}\right)+\frac{\nu_{2}(D)}{2}+1}\right)$. The claim (4.31) is proved. It follows from (4.28)-(4.31) that $\nu_{2}\left(B_{k}\right)=\frac{\nu_{2}(D)}{2}+\max _{\substack{1 \leq i \leq k \\ \nu_{2}(i)=\nu_{2}(D) / 2}}\left\{\nu_{2}\left(a^{2} i^{2}-D\right)\right\}$. On the other hand, since $\nu_{2}\left(a^{2} i^{2}-\right.$ $D)<\nu_{2}(D)$ if $\nu_{2}(i)<\frac{\nu_{2}(D)}{2}$ and $\nu_{2}\left(a^{2} i^{2}-D\right)=\nu_{2}(D)$ if $\nu_{2}(i)>\frac{\nu_{2}(D)}{2}$, we have $\max _{1 \leq i \leq k}\left\{\nu_{2}\left(a^{2} i^{2}-D\right)\right\}=\max \underset{\substack{1 \leq i \leq k \\ \nu_{2}(i)=\nu_{2}(D) / 2}}{1}\left\{\nu_{2}\left(a^{2} i^{2}-D\right)\right\}$. Hence (4.27) follows immediately. Lemma 4.5 is true for Case 3 .

This ends the proof of Lemma 4.5.

Lemma 4.6. Let $\mathcal{K}_{f}$ be nonempty. Then for any $k \in \mathcal{K}_{f}$ and any odd prime $p$ with $p \nmid a$, we have

$$
P_{p, k, f}=\left\{\begin{aligned}
p^{\nu_{p}\left(B_{k}\right)-2 \nu_{p}\left(L_{k}\right)}, & \text { if } k<p^{\left\lceil\frac{\nu_{p}(D)}{2}\right\rceil} \text { and } \nu_{p}(k+1)<\nu_{p}\left(L_{k}\right), \\
p^{\left\lceil\frac{\nu_{p}(D)}{2}\right\rceil}, & \text { if } k \geq p^{\left\lceil\frac{\nu_{p}(D)}{2}\right\rceil}, \nu_{p}(k+1)<\left\lceil\frac{\nu_{p}(D)}{2}\right\rceil \\
& \text { and either } 2 \nmid \nu_{p}(D) \text { or }\left(\frac{D_{p}}{p}\right)=-1, \\
p^{\nu_{p}\left(B_{k}\right)-\nu_{p}(D)}, & \text { if } k \geq p^{\left\lceil\frac{\nu_{p}(D)}{2}\right\rceil}, \nu_{p}(k+1)<\nu_{p}\left(B_{k}\right)-\nu_{p}(D), \\
& 2 \mid \nu_{p}(D) \text { and }\left(\frac{D_{p}}{p}\right)=1, \\
1, & \text { otherwise. }
\end{aligned}\right.
$$

Proof. By Lemma 3.12, we can find a unique nonnegative integer $e$ such that $d_{p^{e}} \leq k<$ $d_{p^{e+1}}$ since $\mathcal{K}_{f}$ is nonempty and $k \in \mathcal{K}_{f}$. Let $p$ be an odd prime with $p \nmid a$. Then

$$
\nu_{p}\left(B_{k}\right)=\nu_{p}\left(\operatorname{lcm}_{1 \leq i \leq k}\left\{i\left(a^{2} i^{2}-D\right)\right\}\right)=\max _{1 \leq i \leq k}\left\{\nu_{p}\left(i\left(a^{2} i^{2}-D\right)\right)\right\} .
$$

If $\left.k<p^{\frac{\Gamma \nu_{p}(D)}{2}}\right\rceil$, then for any integer $i$ with $1 \leq i \leq k$, we have

$$
2 \nu_{p}(i) \leq 2 \nu_{p}\left(L_{k}\right) \leq 2\left(\left\lceil\frac{\nu_{p}(D)}{2}\right\rceil-1\right) \leq \nu_{p}(D)-1,
$$

which implies that $\nu_{p}\left(a^{2} i^{2}-D\right)=2 \nu_{p}(i)$. Hence

$$
\max _{1 \leq i \leq k}\left\{\nu_{p}\left(a^{2} i^{2}-D\right)\right\}=\max _{1 \leq i \leq k}\left\{2 \nu_{p}(i)\right\}=2 \nu_{p}\left(L_{k}\right)
$$

and by (4.32), we have $\nu_{p}\left(B_{k}\right)=\max _{1 \leq i \leq k}\left\{\nu_{p}(i)+2 \nu_{p}(i)\right\}=3 \nu_{p}\left(L_{k}\right)$. Since $p \nmid a$, by Lemma 3.13 (ii), we have $e=\max _{1 \leq i \leq k}\left\{\nu_{p}\left(a^{2} i^{2}-D\right)\right\}$. It then follows from (4.33) and (4.34) that $e=2 \nu_{p}\left(L_{k}\right)<\nu_{p}(D)$. Thus by Lemma 4.4, $P_{p, k, f}=p^{\lceil e / 2\rceil}=p^{\nu_{p}\left(L_{k}\right)}=$ $p^{\nu_{p}\left(B_{k}\right)-2 \nu_{p}\left(L_{k}\right)}$ if $k<p^{\left\lceil\frac{\nu_{p}(D)}{2}\right\rceil}$ and $\nu_{p}(k+1)<\nu_{p}\left(L_{k}\right)$, and $P_{p, k, f}=1$ if $k<p^{\left\lceil\frac{\nu_{p}(D)}{2}\right\rceil}$ 
and $\nu_{p}(k+1) \geq \nu_{p}\left(L_{k}\right)$. So Lemma 4.6 is true if $k<p^{\left\lceil\frac{\nu_{p}(D)}{2}\right\rceil}$. In what follows we let $k \geq p^{\left\lceil\frac{\nu_{p}(D)}{2}\right\rceil}$. Then $\nu_{p}\left(L_{k}\right) \geq\left\lceil\frac{\nu_{p}(D)}{2}\right\rceil$.

If $2 \nmid \nu_{p}(D)$ or $\left(\frac{D_{p}}{p}\right)=-1$, then by parts (i) and (ii) of Lemma 3.10, we have $d_{p^{\nu p(D)}}=$ $p^{\left\lceil\frac{\nu_{p}(D)}{2}\right\rceil}$ and $d_{p^{\nu p(D)+1}}=\infty$. Since $k \geq p^{\left\lceil\frac{\nu_{p}(D)}{2}\right\rceil}$ and $d_{p^{e}} \leq k<d_{p^{e+1}}$, we obtain by Lemma 3.7 that $e=\nu_{p}(D)$. It follows from Lemma 4.4 that $P_{p, k, f}=p^{\lceil e / 2\rceil}=p^{\left\lceil\frac{\nu_{p}(D)}{2}\right\rceil}$ if $\nu_{p}(k+1)<\left\lceil\frac{\nu_{p}(D)}{2}\right\rceil$ and $P_{p, k, f}=1$ if $\nu_{p}(k+1) \geq\left\lceil\frac{\nu_{p}(D)}{2}\right\rceil$. Thus Lemma 4.6 is true if either $k \geq p^{\left\lceil\frac{\nu_{p}(D)}{2}\right\rceil}$ and $2 \nmid \nu_{p}(D)$, or $k \geq p^{\left\lceil\frac{\nu_{p}(D)}{2}\right\rceil}$ and $\left(\frac{D_{p}}{p}\right)=-1$.

If $2 \mid \nu_{p}(D)$ and $\left(\frac{D_{p}}{p}\right)=1$, then $\left\lceil\frac{\nu_{p}(D)}{2}\right\rceil=\frac{\nu_{p}(D)}{2}$ and so $\nu_{p}\left(L_{k}\right) \geq \frac{\nu_{p}(D)}{2}$. First we claim that

$$
\nu_{p}\left(B_{k}\right)=\nu_{p}(D) / 2+\max _{\substack{1 \leq i \leq k \\ \nu_{p}(i)=\nu_{p}(D) / 2}}\left\{\nu_{p}\left(a^{2} i^{2}-D\right)\right\} .
$$

Let $C_{1}:=\max _{\substack{1 \leq i \leq k \\ \nu_{p}(i)=\nu_{p}(D) / 2}}\left\{\nu_{p}\left(i\left(a^{2} i^{2}-D\right)\right)\right\}$ and $C_{2}:=\max _{\nu_{p}(i)>\nu_{p}(\leq) \leq k}\left\{\nu_{p}\left(i\left(a^{2} i^{2}-D\right)\right)\right\}$. Since $C_{1} \geq \frac{3 \nu_{p}(D)}{2}$ and $\nu_{p}\left(i\left(a^{2} i^{2}-D\right)\right)<\frac{3 \nu_{p}(D)}{2}$ if $\nu_{p}(i)<\frac{\nu_{p}(D)}{2}$, we have by (4.32) that

$$
\nu_{p}\left(B_{k}\right)=\max \left(C_{1}, C_{2}\right) .
$$

It also implies that $\nu_{p}\left(B_{k}\right) \geq \frac{3 \nu_{p}(D)}{2}$.

Note that $C_{2}=\max \underset{\nu_{p}(i)>\nu_{p}(\leq) / 2}{1 \leq k}\left\{\nu_{p}(i)\right\}+\nu_{p}(D)=\nu_{p}\left(L_{k}\right)+\nu_{p}(D)$ if $\nu_{p}\left(L_{k}\right)>\frac{\nu_{p}(D)}{2}$. Thus by (4.36), we obtain that

$$
\nu_{p}\left(B_{k}\right)=\max \left(C_{1}, \nu_{p}\left(L_{k}\right)+\nu_{p}(D)\right)
$$

if $\nu_{p}\left(L_{k}\right)>\frac{\nu_{p}(D)}{2}$. If $\nu_{p}\left(L_{k}\right)>\frac{\nu_{p}(D)}{2}$, since the discriminant of $a^{2} x^{2}-D$ is $4 a^{2} D$ and $\nu_{p}\left(4 a^{2} D\right)=\nu_{p}(D)$, applying Lemma 3.3 (iii) to the congruence $a^{2} x^{2}-D \equiv 0$ $\left(\bmod p^{\nu_{p}\left(L_{k}\right)+\frac{\nu_{p}(D)}{2}}\right)$, we know that there is an integer $x_{0} \in\left[1, p^{\nu_{p}\left(L_{k}\right)}\right] \subseteq[1, k]$ such that $\nu_{p}\left(x_{0}\right)=\frac{\nu_{p}(D)}{2}$ and $\nu_{p}\left(a^{2} x_{0}^{2}-D\right) \geq \nu_{p}\left(L_{k}\right)+\frac{\nu_{p}(D)}{2}$. Hence

$$
C_{1}=\frac{\nu_{p}(D)}{2}+\max _{\substack{1 \leq i \leq k \\ \nu_{p}(i)=\nu_{p}(D) / 2}}\left\{\nu_{p}\left(a^{2} i^{2}-D\right)\right\} \geq \nu_{p}\left(L_{k}\right)+\nu_{p}(D) .
$$

It then follows from (4.37) that $\nu_{p}\left(B_{k}\right)=C_{1}$ if $\nu_{p}\left(L_{k}\right)>\frac{\nu_{p}(D)}{2}$. On the other hand, there is no integer $i \in[1, k]$ such that $\nu_{p}(i)>\frac{\nu_{p}(D)}{2}$ if $\nu_{p}\left(L_{k}\right)=\frac{\nu_{p}(D)}{2}$. So by (4.36), $\nu_{p}\left(B_{k}\right)=C_{1}$ if $\nu_{p}\left(L_{k}\right)=\frac{\nu_{p}(D)}{2}$. Thus $\nu_{p}\left(B_{k}\right)=C_{1}$ if $k \geq p^{\left\lceil\frac{\nu_{p}(D)}{2}\right\rceil}$ and $2 \mid \nu_{p}(D)$ and $\left(\frac{D_{p}}{p}\right)=1$. The claim (4.35) is proved.

One can easily check that $\max _{1 \leq i \leq k}\left\{\nu_{p}\left(a^{2} i^{2}-D\right)\right\}=\max \underset{\substack{1 \leq i \leq k \\ \nu_{p}(i)=\nu p(D) / 2}}{ }\left\{\nu_{p}\left(a^{2} i^{2}-D\right)\right\}$. It then follows from (4.35) that $\nu_{p}\left(B_{k}\right)=\frac{\nu_{p}(D)}{2}+\max _{1 \leq i \leq k}\left\{\nu_{p}\left(a^{2} i^{2}-D\right)\right\}$. Hence by Lemma 3.13 (ii), we have $e=\max _{1 \leq i \leq k}\left\{\nu_{p}\left(a^{2} i^{2}-D\right)\right\}=\nu_{p}\left(B_{k}\right)-\frac{\nu_{p}(D)}{2}$, which implies that $e-\frac{\nu_{p}(D)}{2}=\nu_{p}\left(B_{k}\right)-\nu_{p}(D) \geq \frac{\nu_{p}(D)}{2}$ and so $e \geq \nu_{p}(D)$. Also we have $\lceil e / 2\rceil=$ $\nu_{p}(D) / 2=e-\nu_{p}(D) / 2=\nu_{p}\left(B_{k}\right)-\nu_{p}(D)$ if $e=\nu_{p}(D)$. It then follows from Lemma 4.4 that $P_{p, k, f}=p^{\nu_{p}\left(B_{k}\right)-\nu_{p}(D)}$ if $\nu_{p}(k+1)<\nu_{p}\left(B_{k}\right)-\nu_{p}(D)$ and $P_{p, k, f}=1$ if $\nu_{p}(k+1) \geq$ $\nu_{p}\left(B_{k}\right)-\nu_{p}(D)$. So Lemma 4.6 is true if $k \geq p^{\left\lceil\frac{\nu_{p}(D)}{2}\right\rceil}, 2 \nmid \nu_{p}(D)$ and $\left(\frac{D_{p}}{p}\right)=1$. This completes the proof of Lemma 4.6. 


\section{Proof of Theorem 1.2 and examples}

In this section, we first give the proof of Theorem 1.2 by using Lemmas $2.2,4.2,4.5$ and 4.6 .

Proof of Theorem 1.2. Since $g_{k, f}(n)=g_{k,-f}(n)$ for any $n \in \mathbb{N}^{*} \backslash Z_{k, f}$, we can assume that $a>0$ in the following. If $f(x)=a x^{2}+b x+c$ satisfies that $\operatorname{gcd}(a, b, c)=d>1$, we can then easily get that

$$
g_{k, f}(n)=\frac{\prod_{i=0}^{k}|f(n+i)|}{\operatorname{lcm}_{0 \leq i \leq k}\{f(n+i)\}}=d^{k} \frac{\prod_{i=0}^{k} f_{1}(n+i)}{\operatorname{lcm}_{0 \leq i \leq k}\left\{f_{1}(n+i)\right\}}=d^{k} g_{k, f_{1}},
$$

where $f_{1}(x)=a_{1} x^{2}+b_{1} x+c_{1}$ with $a_{1}=a / d, b_{1}=b / d$ and $c_{1}=c / d$. Obviously, $g_{k, f}$ and $g_{k, f_{1}}$ have the same periodicity. If they are both periodic, they have the same smallest period. That is, we have $P_{k, f}=P_{k, f_{1}}$. Therefore we assume that $f(x)$ is a primitive polynomial (i.e., $\operatorname{gcd}(a, b, c)=1)$ in what follows.

Since $f$ is primitive, by Theorem 2.1 we know that the first part of Theorem 1.2 is true. Now we assume that $D \neq a^{2} i^{2}$ for all integers $i$ with $1 \leq i \leq k$. Then $g_{k, f}$ is eventually periodic by Theorem 2.1. Note that $\mathcal{K}_{f}$ is nonempty and $k \in \mathcal{K}_{f}$. In what follows we determine the smallest period $P_{k, f}$ of $g_{k, f}$. Let $\Delta_{p, k}:=\nu_{p}\left(B_{k}\right)-\nu_{p}\left(P_{p, k, f}\right)$ for any prime $p$. Since by Lemma $2.2, P_{p, k, f} \mid p^{\nu_{p}\left(B_{k}\right)}$ for any prime $p$. Hence $P_{2, k, f}=1$ if $2 \nmid B_{k}$. So again by Lemma 2.2, we can derive that

$$
P_{k, f}=P_{2, k, f} \prod_{p \neq 2, p \mid B_{k}} p^{\nu_{p}\left(P_{p, k, f}\right)}=\frac{B_{k}}{2^{\Delta_{2, k}} \prod_{p \neq 2, p \mid B_{k}} p^{\Delta_{p, k}}}=\frac{B_{k}}{E_{k} F_{k}},
$$

where

$$
E_{k}:=2^{\Delta_{2, k}}\left(\prod_{p \neq 2, p \mid \operatorname{gcd}(a, b)} p^{\Delta_{p, k}}\right)\left(\prod_{p \nmid 2 a, p \mid D} p^{\Delta_{p, k}}\right)\left(\prod_{p \nmid 2 a D,\left(\frac{D}{p}\right)=-1} p^{\Delta_{p, k}}\right)
$$

and

$$
F_{k}:=\left(\prod_{p \mid a, p \nmid 2 b} p^{\Delta_{p, k}}\right)\left(\prod_{p \nmid 2 a D,\left(\frac{D}{p}\right)=1} p^{\Delta_{p, k}}\right) .
$$

First we treat with $E_{k}$. If $p=2$, then we get by Lemmas 4.2 and 4.5 that

$$
\nu_{2}\left(P_{2, k, f}\right)=\left\{\begin{aligned}
\nu_{2}\left(B_{k}\right), & \text { if } 2 \mid a, 2 \nmid b \text { and } \nu_{2}(k+1)<\nu_{2}\left(B_{k}\right), \\
\nu_{2}\left(B_{k}\right)-2 \nu_{2}\left(L_{k}\right), & \text { if } 2 \nmid a, k<2^{\left\lfloor\frac{\nu_{2}(D)}{2}\right\rfloor} \text { and } \nu_{2}(k+1)<\nu_{2}\left(L_{k}\right), \\
\left\lfloor\frac{\nu_{2}(D)}{2}\right\rfloor, & \text { if } 2 \nmid a, k \geq 2^{\left\lfloor\frac{\nu_{2}(D)}{2}\right\rfloor}, D_{4} \not \equiv 1 \quad(\bmod 8) \\
& \text { and } \nu_{2}(k+1)<\left\lfloor\left\lfloor\frac{\nu_{2}(D)}{2}\right\rfloor,\right. \\
\nu_{2}\left(B_{k}\right)-\nu_{2}(D)-1, & \text { if } 2 \nmid a, k \geq 2^{\left\lfloor\frac{\nu_{2}(D)}{2}\right\rfloor} \text { and } D_{4} \equiv 1 \quad(\bmod 8), \\
0, & \text { otherwise. }
\end{aligned}\right.
$$

Thus $2^{\Delta_{2, k}}=\xi_{2}$ with $\xi_{2}$ being defined in (1.3).

If $p \neq 2$ and $p \mid \operatorname{gcd}(a, b)$, then by Lemma 4.2 , we have $\nu_{p}\left(P_{p, k, f}\right)=0$ and so $\Delta_{p, k}=$ $\nu_{p}\left(B_{k}\right)$. Hence

$$
\prod_{p \neq 2, p \mid \operatorname{gcd}(a, b)} p^{\Delta_{p, k}}=\prod_{p \neq 2, p \mid \operatorname{gcd}(a, b)} p^{\nu_{p}\left(B_{k}\right)} .
$$


If $p \nmid 2 a$ and $p \mid D$, then using Lemma 4.6, we obtain

$$
\Delta_{p, k}=\left\{\begin{aligned}
2 \nu_{p}\left(L_{k}\right), & \text { if } k<p^{\left\lceil\frac{\nu_{p}(D)}{2}\right\rceil} \text { and } \nu_{p}(k+1)<\nu_{p}\left(L_{k}\right), \\
\nu_{p}\left(B_{k}\right)-\left\lceil\frac{\nu_{p}(D)}{2}\right\rceil, & \text { if } k \geq p^{\left\lceil\frac{\nu_{p}(D)}{2}\right\rceil}, \nu_{p}(k+1)<\left\lceil\frac{\nu_{p}(D)}{2}\right\rceil \\
& \text { and either } 2 \nmid \nu_{p}(D) \text { or }\left(\frac{D_{p}}{p}\right)=-1, \\
\nu_{p}(D), & \text { if } k \geq p^{\left\lceil\frac{\nu_{p}(D)}{2}\right\rceil}, \nu_{p}(k+1)<\nu_{p}\left(B_{k}\right)-\nu_{p}(D), \\
& 2 \mid \nu_{p}(D) \text { and }\left(\frac{D_{p}}{p}\right)=1, \\
\nu_{p}\left(B_{k}\right), & \text { otherwise. }
\end{aligned}\right.
$$

It follows that

$$
\prod_{p \nmid 2 a, p \mid D} p^{\Delta_{p, k}}=\prod_{p \nmid 2 a, p \mid D} \eta_{p}
$$

where $\eta_{p}$ is defined as in (1.4).

If $p \nmid 2 a D$ and $\left(\frac{D}{p}\right)=-1$, then $\nu_{p}(D)=0$ and $D=D_{p}$. Hence we have $k \geq$ $p^{\left\lceil\frac{\nu_{p}(D)}{2}\right\rceil}=1, \nu_{p}(k+1) \geq\left\lceil\frac{\nu_{p}(D)}{2}\right\rceil$ and $\left(\frac{D_{p}}{p}\right)=-1$. It then follows from Lemma 4.6 that $\nu_{p}\left(P_{p, k, f}\right)=0$, which implies that $\Delta_{p, k}=\nu_{p}\left(B_{k}\right)$. Therefore

$$
\prod_{p \nmid 2 a D,\left(\frac{D}{p}\right)=-1} p^{\Delta_{p, k}}=\prod_{p \nmid 2 a D,\left(\frac{D}{p}\right)=-1} p^{\nu_{p}\left(B_{k}\right)} .
$$

Now by (5.2) and (5.4)-(5.6), we get that $E_{k}=\frac{B_{k}}{A_{k}}$, where $A_{k}$ is defined in (1.2). Thus by (5.1), we have $P_{k, f}=\frac{A_{k}}{F_{k}}$.

Consequently, we handle $F_{k}$. For this purpose, we first prove the following fact: There is at most one prime $p$ such that $\nu_{p}(k+1) \geq \nu_{p}\left(B_{k}\right) \geq 1$. Suppose that there are two distinct primes $p_{1}$ and $p_{2}$ such that $v_{p_{1}}(k+1) \geq v_{p_{1}}\left(B_{k}\right) \geq 1$ and $v_{p_{2}}(k+1) \geq v_{p_{2}}\left(B_{k}\right) \geq 1$. Then $k+1$ is composite and so $p_{1} \leq k$ and $p_{2} \leq k$. Hence for each $1 \leq j \leq 2$,

$$
v_{p_{j}}(k+1) \geq v_{p_{j}}\left(B_{k}\right)=\max _{1 \leq i \leq k}\left\{v_{p_{j}}(i)+v_{p_{j}}\left(a^{2} i^{2}-D\right)\right\} \geq \max _{1 \leq i \leq k}\left\{v_{p_{j}}(i)\right\}=v_{p_{j}}\left(L_{k}\right) \geq 1 .
$$

But Farhi and Kane [7] showed that there is at most one prime $p \leq k$ such that $\nu_{p}(k+1) \geq$ $\nu_{p}\left(L_{k}\right) \geq 1$. We arrive at a contradiction. Thus the fact is proved.

Now we turn to $F_{k}$. Let $p \mid B_{k}$ be a prime satisfying that either $p \mid a$ and $p \nmid 2 b$ or $p \nmid 2 a D$ and $\left(\frac{D}{p}\right)=1$. Then $\nu_{p}\left(A_{k}\right)=\nu_{p}\left(B_{k}\right)$. It then follows from the above fact that there is at most one prime $p$ such that $\nu_{p}(k+1) \geq \nu_{p}\left(A_{k}\right) \geq 1$.

For any prime $p$ satisfying that either $p \mid a, p \nmid 2 b$ and $\nu_{p}(k+1)<\nu_{p}\left(B_{k}\right)$, or $p \nmid$ $2 a D,\left(\frac{D}{p}\right)=1$ and $\nu_{p}(k+1)<\nu_{p}\left(B_{k}\right)$, by Lemmas 4.2 and 4.6 , we deduce that $\nu_{p}\left(P_{p, k, f}\right)=$ $\nu_{p}\left(B_{k}\right)$ and so $\Delta_{p, k}=0$. If there is no prime $p$ satisfying that $\nu_{p}(k+1) \geq \nu_{p}\left(A_{k}\right) \geq 1$ and either $p \mid a$ and $p \nmid b$ or $p \nmid 2 a D$ and $\left(\frac{D}{p}\right)=1$, it then follows from (5.3) that $F_{k}=1$.

If there is exactly one odd prime $q$ satisfying that $v_{q}(k+1) \geq v_{q}\left(A_{k}\right) \geq 1$ and either $q \mid a$ and $q \nmid b$ or $q \nmid 2 a D$ and $\left(\frac{D}{q}\right)=1$, then $v_{q}\left(P_{q, k, f}\right)=0$. Thus $F_{k}=q^{v_{q}\left(B_{k}\right)}=q^{v_{q}\left(A_{k}\right)}$. Since $P_{k, f}=\frac{A_{k}}{F_{k}}$, one concludes that $P_{k, f}=A_{k}$ except that $v_{q}(k+1) \geq v_{q}\left(A_{k}\right) \geq 1$ for at most one odd prime $q$ such that either $q \mid a$ and $q \nmid b$ or $q \nmid 2 a D$ and $\left(\frac{D}{q}\right)=1$, in which case one has $P_{k, f}=A_{k} / q^{v_{q}\left(A_{k}\right)}$. The proof of Theorem 1.2 is complete.

Now we give two examples to illustrate Theorem 1.2 .

Example 5.1. Let $f(x)=4^{l} x^{2}+1$ with $l \geq 1$ being an integer. Then $D=-4^{l+1}$, $\mathcal{K}_{f}=\mathbb{N}^{*}$ and $Z_{k, f}$ is empty for all integers $k \geq 1$. By Theorem 1.2, $g_{k, f}$ is periodic for all integers $k \geq 1$. We have by (1.4), $B_{k}:=\operatorname{lcm}_{1 \leq i \leq k}\left\{i\left(16^{l} i^{2}+4^{l+1}\right)\right\}$. Since 
$2 \mid 4^{l}=\operatorname{gcd}(a, b)$, we obtain $\xi_{2}=2^{\nu_{2}\left(B_{k}\right)}$ by (1.3). Clearly, there is no odd prime $p$ such that $p \mid \operatorname{gcd}(a, b)$ or $p \mid D$. On the other hand, all the primes satisfying $\left(\frac{-4^{l+1}}{p}\right)=1$ are of the form $p \equiv 1(\bmod 4)$, and all the primes such that $\left(\frac{-4^{l+1}}{p}\right)=-1$ must be of the form $p \equiv 3(\bmod 4)$. Hence by (1.2), we have $A_{k}:=B_{k} 2^{-\nu_{2}\left(B_{k}\right)} \prod_{p \equiv 3(\bmod 4)} p^{-\nu_{p}\left(B_{k}\right)}$. By Theorem 1.2. the smallest period of $g_{k, f}$ equals $A_{k}$ except that $\nu_{p}(k+1) \geq \nu_{p}\left(A_{k}\right) \geq 1$ for at most one prime $p \equiv 1(\bmod 4)$, in which case its smallest period is equal to $\frac{A_{k}}{p^{\nu p\left(A_{k}\right)}}$. Example 5.2. Let $f(x)=(x+m)(x+m+l)$ with $m \geq 0$ and $l \geq 2$ being integers. Then $D=l^{2}$ and $\mathcal{K}_{f}=\{1, \ldots, l-1\}$. By Theorem $1.2, g_{k, f}$ is eventually periodic if and only if $1 \leq k \leq l-1$. Now let $1 \leq k \leq l-1$. Then by (1.4), $B_{k}=\operatorname{lcm}_{1 \leq i \leq k}\left\{i\left(a^{2} i^{2}-D\right)\right\}=$ $\operatorname{lcm}_{1 \leq i \leq k}\left\{i\left(l^{2}-i^{2}\right)\right\}$. Since $D_{4}=\frac{l^{2}}{2^{2 \nu_{2}(l)}} \equiv 1(\bmod 8)$ and $v_{q}(D)=2 v_{q}(l)$ for any prime factor $q$ of $D$, we have by (1.3) and (1.4) that

$$
\xi_{2}=\left\{\begin{array}{cl}
2^{2 \nu_{2}\left(L_{k}\right)}, & \text { if } k<2^{\nu_{2}(l)} \text { and } \nu_{2}(k+1)<\nu_{2}\left(L_{k}\right), \\
2^{\nu_{2}\left(B_{k}\right)}, & \text { if } k<2^{\nu_{2}(l)} \text { and } \nu_{2}(k+1) \geq \nu_{2}\left(L_{k}\right), \\
2^{2 \nu_{2}(l)+1}, & \text { if } k \geq 2^{\nu_{2}(l)}
\end{array}\right.
$$

and

$$
\eta_{p}=\left\{\begin{array}{cl}
p^{2 \nu_{p}\left(L_{k}\right)}, & \text { if } k<p^{\nu_{p}(l)} \text { and } \nu_{p}(k+1)<\nu_{p}\left(L_{k}\right), \\
p^{2 \nu_{p}(l)}, & \text { if } k \geq p^{\nu_{p}(l)} \text { and } \nu_{p}(k+1)<\nu_{p}\left(B_{k}\right)-2 \nu_{p}(l), \\
p^{\nu_{p}\left(B_{k}\right)}, & \text { otherwise. }
\end{array}\right.
$$

Moreover, $\left(\frac{D}{p}\right)=\left(\frac{l^{2}}{p}\right)=1$ for any prime $p$ with $p \nmid 2 a D$ and there is no odd prime $p$ such that $p \mid \operatorname{gcd}(a, b)$. It then follows from (1.2) that $A_{k}=B_{k} \xi_{2}^{-1} \prod_{p \neq 2, p \mid l} \eta_{p}^{-1}$. Thus by Theorem 1.2. the smallest period of $g_{k, f}$ is equal to $A_{k}$ except that $\nu_{p}(k+1) \geq \nu_{p}\left(A_{k}\right) \geq 1$ for at most one odd prime $p$ with $p \nmid 2 D$, in which case its smallest period equals $\frac{A_{k}}{p^{\nu_{p}\left(A_{k}\right)}}$.

\section{Proof of Theorem 1.3}

In this section, we show Theorem 1.3 .

Proof of Theorem 1.3. It is clear that if $\operatorname{gcd}(a, b, c)=d$, then $\log \operatorname{lcm}_{0<i<k}\{f(n+i)\}=$ $\log \operatorname{lcm}_{0 \leq i \leq k}\left\{f_{1}(n+i)\right\}+O(1)$, where $f_{1}(x)=f(x) / d$ is a primitive polynomial. So without loss of generality, we assume that $\operatorname{gcd}(a, b, c)=1$ and $a>0$ in what follows.

(i). Since $D \neq a^{2} i^{2}$ for all $1 \leq i \leq k, \mathcal{K}_{f}$ is nonempty and $k \in \mathcal{K}_{f}$. By Theorem 1.2, $g_{k, f}$ is eventually periodic. So there is a positive integer $n_{0}$ such that for all positive integers $n \geq n_{0}$, we have $g_{k, f}(n) \leq M:=\max _{1 \leq m \leq P_{k, f}}\left\{g_{k, f}\left(n_{0}+m\right)\right\}$. Hence for sufficiently large $n, \log \left(\prod_{i=0}^{k}|f(n+i)|\right)-\log M \leq \log \operatorname{lcm}_{0 \leq i \leq k}\{f(n+i)\} \leq \log \left(\prod_{i=0}^{k}|f(n+i)|\right)$.

Since $\log \left(\prod_{i=0}^{k}|f(n+i)|\right)=2(k+1) \log n+\sum_{i=0}^{k} \log \left(a+\frac{2 a i+b}{n}+\frac{a i^{2}+b i+c}{n^{2}}\right)$ for sufficiently large $n$, one has $\lim _{n \rightarrow \infty} \frac{\log \left(\prod_{i=0}^{k}|f(n+i)|\right)}{2(k+1) \log n}=1$ and $\lim _{n \rightarrow \infty} \frac{\log \left(\prod_{i=0}^{k}|f(n+i)|\right)-\log M}{2(k+1) \log n}$ $=1$. This concludes the desired result.

(ii). Since $D=a^{2} i_{0}^{2}$ for some integer $i_{0}$ with $1 \leq i_{0} \leq k, f(x)$ is reducible. It then follows from the proof of Theorem 2.1 that $f(x)$ must be of the form $\left(a_{1} x+b_{1}\right)\left(a_{1} x+b_{1}+\right.$ $\left.a_{1} i_{0}\right)$ for some integers $a_{1}>0$ and $b_{1}$ with $\operatorname{gcd}\left(a_{1}, b_{1}\right)=1$. Thus $\operatorname{lcm}_{0 \leq i \leq k}\{f(n+i)\}=$ $\operatorname{lcm}_{0 \leq i \leq k}\left\{\left(a_{1}(n+i)+b_{1}\right)\left(a_{1}\left(n+i+i_{0}\right)+b_{1}\right)\right\}$. It is easy to see that $\left\{a_{1}(x+i)+b_{1}\right\}_{0 \leq i \leq k+i_{0}}$ is equal to the set of all the linear factors of $\prod_{i=0}^{k} f(x+i)$. Hence $\operatorname{lcm}_{0 \leq i \leq k+i_{0}}\left\{a_{1}(n+\right.$ $\left.i)+b_{1}\right\}$ divides $\operatorname{lcm}_{0 \leq i \leq k}\{f(n+i)\}$. So we get that $\operatorname{lcm}_{0 \leq i \leq k+i_{0}}\left\{a_{1}(n+i)+b_{1}\right\} \leq$ $\operatorname{lcm}_{0 \leq i \leq k}\{f(n+i)\} \leq \prod_{0 \leq i \leq k+i_{0}}\left(a_{1}(n+i)+b_{1}\right)$ for sufficiently large integer $n$. 
If $b_{1} \geq 0$, then as in [12, we define the following arithmetic function $g_{k+i_{0}, a_{1}, b_{1}}(n):=$ $\frac{\prod_{0 \leq i \leq k+i_{0}}\left(a_{1}(n+i)+b_{1}\right)}{\operatorname{lcm}_{0 \leq i \leq k+i_{0}}\left\{a_{1}(n+i)+b_{1}\right\}}$. Then by Theorem 1.3] of [12], $g_{k+i_{0}, a_{1}, b_{1}}$ is a periodic arithmetic function. So there is a fixed positive integer $M$ such that $g_{k+i_{0}, a_{1}, b_{1}}(n) \leq M$ for all positive integers $n$. If $b_{1}<0$, then we make a revision to the above argument by defining $\tilde{g}_{k+i_{0}, a_{1}, b_{1}}$ as follows $\tilde{g}_{k+i_{0}, a_{1}, b_{1}}(n):=g_{k+i_{0}, a_{1}, b_{1}}\left(n-b_{1}\right)$. Then Theorem 1.3 of [12] tells us that $\tilde{g}_{k+i_{0}, a_{1}, b_{1}}$ is a periodic arithmetic function. Thus there exists a fixed positive integer $M$ such that $\tilde{g}_{k+i_{0}, a_{1}, b_{1}}(n) \leq M$ for all positive integers $n$. So $g_{k+i_{0}, a_{1}, b_{1}}(n) \leq M$ for all positive integers $n \geq-b_{1}$. This concludes that $g_{k+i_{0}, a_{1}, b_{1}}(n) \leq M$ for all sufficiently large integers $n$. Thus we obtain that $\frac{\prod_{0 \leq i \leq k+i_{0}}\left(a_{1}(n+i)+b_{1}\right)}{M} \leq \operatorname{lcm}_{0 \leq i \leq k}\{f(n+i)\} \leq$ $\prod_{0 \leq i \leq k+i_{0}}\left(a_{1}(n+i)+b_{1}\right)$ for sufficiently large $n$. Since $\lim _{n \rightarrow \infty} \frac{\log \prod_{0 \leq i \leq k+i_{0}}\left(a_{1}(n+i)+b_{1}\right)}{\left(k+i_{0}+1\right) \log n}=$ 1 , the desired result then follows immediately. So Theorem 1.3 is proved.

Acknowledgements. The authors would like to thank Professor Freydoon Shahidi and the anonymous referee for helpful comments and suggestions which improved its presentation.

\section{REFERENCES}

[1] T.M. Apostol, Introduction to analytic number theory, Springer-Verlag, New York, 1976.

[2] P. Bateman, J. Kalb and A. Stenger, A limit involving least common multiples, Amer. Math. Monthly 109 (2002), 393-394.

[3] P.L. Chebyshev, Memoire sur les nombres premiers, J. Math. Pures Appl. 17 (1852), 366-390.

[4] W. Duke, J. Friedlander and H. Iwaniec, Equidistribution of roots of a quadratic congruence to prime moduli, Ann. Math. (2) 141 (1995), 423-441.

[5] B. Farhi, Minoration non triviales du plus petit commun multiple de certaines suites finies d'entiers, C. R. Acad. Sci. Paris Sér. I 341 (2005), 469-474.

[6] B. Farhi, Nontrivial lower bounds for the least common multiple of some finite sequences of integers, J. Number Theory 125 (2007), 393-411.

[7] B. Farhi and D. Kane, New results on the least common multiple of consecutive integers, Proc. Amer. Math. Soc. 137 (2009), 1933-1939.

[8] D. Hanson, On the product of the primes, Canad. Math. Bull. 15 (1972), 33-37.

[9] S. Hong, The least common multiple of consecutive terms in a sequence of positive integers, Proceedings of Sixth International Congress of Chinese Mathematicians. AMS/IP Stud. Adv. Math., Amer. Math. Soc., Providence, RI, to appear in 2014.

[10] S. Hong and W. Feng, Lower bounds for the least common multiple of finite arithmetic progressions, C.R. Acad. Sci. Paris, Ser. I 343 (2006), 695-698.

[11] S. Hong, Y. Luo, G. Qian and C. Wang, Uniform lower bound for the least common multiple of a polynomial sequence, C.R. Acad. Sci. Paris, Ser. I 351 (2013), 781-785.

[12] S. Hong and G. Qian, The least common multiple of consecutive arithmetic progression terms, Proc. Edinburgh Math. Soc. 54 (2011), 431-441.

[13] S. Hong, G. Qian and Q. Tan, The least common multiple of a sequence of products of linear polynomials, Acta Math. Hungar. 135 (2012), 160-167.

[14] S. Hong and Y. Yang, On the periodicity of an arithmetical function, C.R. Acad. Sci. Paris Sér. I 346 (2008), 717-721.

[15] S. Hong and Y. Yang, Improvements of lower bounds for the least common multiple of finite arithmetic progressions, Proc. Amer. Math. Soc. 136 (2008), 4111-4114.

[16] L.-K. Hua, Introduction to number theory, Springer-Verlag, Berlin Heidelberg, 1982.

[17] D.M. Kane and S.D. Kominers, Asymptotic improvements of lower bounds for the least common multiples of arithmetic progressions, Canad. Math. Bull., to appear. http://math.stanford.edu/ dankane/lcmbound.pdf

[18] N. Koblitz, $p$-Adic numbers, $p$-adic analysis and zeta functions, GTM 58, Spinger-Verlag, New York, 1984.

[19] P.J. McCarthy, Introdcution to arithmetical functions, Springer-Verlag, New York, 1982.

[20] M. Nair, On Chebyshev-type inequalities for primes, Amer. Math. Monthly 89 (1982), 126-129. 
[21] S.M. Oon, Note on the lower bound of least common multiple, Abstr. Appl. Anal. 2013, Art. ID 218125, 4 pp.

[22] G. Qian and S. Hong, Asymptotic behavior of the least common multiple of consecutive arithmetic progression terms, Arch. Math. 100 (2013), 337-345.

[23] G. Qian, Q. Tan and S. Hong, The least common multiple of consecutive terms in a quadratic progression, Bull. Aust. Math. Soc. 86 (2012), 389-404.

[24] R. Wu, Q. Tan and S. Hong, New lower bounds for the least common multiples of arithmetic progressions, Chin. Ann. Math. 34B (2013), 861-864.

Mathematical College, Sichuan University, Chengdu 610064, P.R. China

E-mail address: sfhong@scu.edu.cn, s-f.hong@tom.com, hongsf02@yahoo.com

Center for Combinatorics, Nankai University, Tianjin 300071, P.R. China

E-mail address: qiangy1230@163.com, qiangy1230@gmail.com 